Percy Alexander Cáceres Tintaya

\title{
Riemann Hilbert problems in Random Matrix Theory
}

\section{Dissertação de Mestrado}

Thesis presented to the Programa de Pós-Graduação em Física of the Departamento de Física do Centro Técnico Científico da PUC-Rio, as partial fulfillment of the requirements for the degree of Mestre.

Advisor : Prof. Hiroshi Nunokawa Co-Advisor: Prof. Stefan Zohren 


\section{Percy Alexander Cáceres Tintaya}

\section{Riemann Hilbert problems in Random Matrix Theory}

Thesis presented to the Programa de Pós-Graduação em Física of the Departamento de Física do Centro Técnico Científico da PUC-Rio, as partial fulfillment of the requirements for the degree of Mestre.

Prof. Hiroshi Nunokawa

Advisor

Departamento de Física - PUC-Rio

Prof. Stefan Zohren

Co-Advisor

Departamento de Física - PUC-Rio

\section{Prof. Welles Antonio Martinez Morgado}

Departamento de Física - PUC-Rio

Prof. José Abdalla Helayël-Neto

CBPF

Prof. José Eugenio Leal Coordinator of the Centro Técnico Científico - PUC-Rio 
All rights reserved.

\section{Percy Alexander Cáceres Tintaya}

Graduated as Dipl.-Phys. from Universidad Nacional de Ingenieria-Perú.

Cáceres Tintaya, Percy Alexander

Riemann Hilbert problems in Random Matrix Theory / Percy Alexander Cáceres Tintaya; advisor: Hiroshi Nunokawa; co-advisor: Stefan Zohren . - Rio de Janeiro: Departamento de Física, Pontifícia Universidade Católica do Rio de Janeiro, 2015.

126 f. : il. ; $30 \mathrm{~cm}$

1. Dissertação (Mestrado em Física) - Pontifícia Universidade Católica do Rio de Janeiro, Rio de Janeiro, 2015.

Inclui bibliografia

1. Física - Teses. Teoria das Matrizes Aleatórias; Emsemble Unitário Gaussiano; Gas de Dyson; Polinômios ortogonais; Problemas de Riemann-Hilbert; Método de máxima gradiente. I. Nunokawa, Hiroshi. II. Zohren, Stefan. III. Pontifícia Universidade Católica do Rio de Janeiro. Departamento de Física. IV. Título. 


\section{Acknowledgments}

With my deep respect I would like to thank Prof. Stefan Zohren who has guided me throughout the interesting random matrix world. Also I like to thank Prof. Hiroshi Nunokawa who allowed me to finish this thesis and to thank Rouhollah Ebrahimi and Prof. Welles Antonio Martinez Morgado for making a critical reading of the thesis.

It is my pleasure to thank the Departamento de Fisica in PUC RIO for their support, both financial and infrastructural. It is also a pleasure to thank to $\mathrm{CNPq}$ for the financial support.

My love and appreciation to my mother Paula Tintaya for her constant encouragement.

Last (but certainly not least) I would like to express my gratitude to the Gajokai da BSGI for training me in the life's university. 


\section{Abstract}

Cáceres Tintaya, Percy Alexander; Nunokawa, Hiroshi (advisor); Zohren, Stefan (co-advisor). Riemann Hilbert problems in Random Matrix Theory. Rio de Janeiro, 2015. 126p. MSc. Dissertation - Departamento de Física, Pontifícia Universidade Católica do Rio de Janeiro.

We review the basic notions of the Random Matrix Theory and in particular the Gaussian Unitary Ensemble. In what follows we describe the Dyson gas in equilibrium and nonequilibrium that allows one to interpret the statistical information of the eigenvalues of random matrices. Furthermore we show alternative descriptions of this statistical information. In the following we discuss different aspects of orthogonal polynomials. One of these caracterizations is given by a Riemann Hilbert problem. Riemann Hilbert problem techniques are an efficient and powerfull tool for Random Matrix Theory which we discuss in more detail. In the final part we use the steepest descent method in the asymptotic analysis of orthogonal polynomials.

\section{Keywords}

Random Matrix Theory; Gaussian Unitary Ensemble; Dyson gas; Orthogonal polynomials; Riemann Hilbert problem; Steepest descent method. 


\section{Resumo}

Cáceres Tintaya, Percy Alexander; Nunokawa, Hiroshi (orientador) ; Zohren, Stefan (co-orientador). Problemas de RiemannHilbert na teoria de Matrizes Aleatórias. Rio de Janeiro, 2015. 126f. Dissertação de Mestrado — Departamento de Física, Pontifícia Universidade Católica do Rio de Janeiro.

Estudamos as noções básicas da Teoria das Matrizes Aleatórias e em particular discutimos o Emsemble Unitário Gaussiano. A continuação descrevemos o gaz de Dyson em equilíbrio e fora do equilíbrio que permite interpretar a informação estatística dos autovalores das matrizes aleatórias. Além desso mostramos descrições alternativas dessa informação estatística. Em seguida discutimos aspectos diferentes dos polinômios ortogonais. Uma dessas caracterizações é dada pelos problemas de Riemann-Hilbert. As técnicas dos problemas de Riemann-Hilbert são uma ferramenta eficaz e potente na Teoria das Matrizes Aleatórias a qual discutimos com mais cuidado. Finalmente usamos o método de máxima gradiente na análise assintótico dos polinômios ortogonais.

\section{Palavras-chave}

Teoria das Matrizes Aleatórias; Emsemble Unitário Gaussiano; Gas de Dyson; Polinômios ortogonais; Problemas de Riemann-Hilbert; Método de máxima gradiente. 


\section{Contents}

1 Introduction $\quad 12$

2 Random Matrix Theory $\quad 14$

2.1 Introduction 14

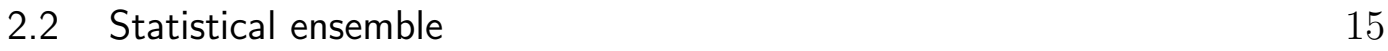

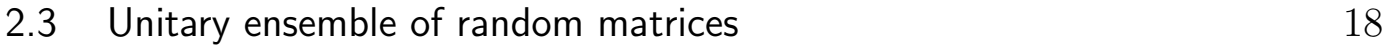

2.4 Ensemble of eigenvalues 21

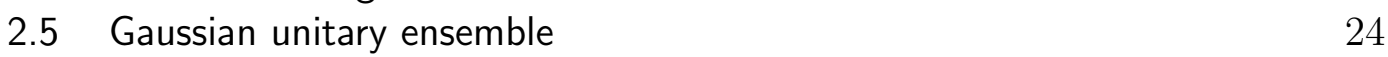

2.6 Statistical properties of the eigenvalues spectrum 27

3 The Dyson gas and eigenvalue density 30

$\begin{array}{lll}3.1 & \text { Introduction } & 30\end{array}$

3.2 Dyson gas 31

3.3 Dyson-Nonstationary ensemble 32

3.4 Saddle point equation 35

3.5 Resolvent function as a Riemann Hilbert representation 37

4 Orthogonal Polynomials $\quad 41$

4.1 Introduction 41

4.2 Generalities of orthogonal polynomials 42

4.3 Analytical Representation 46

4.4 Geometrical Representation $\quad 49$

4.5 Algebraic Representation $\quad 51$

4.6 Statistical Representation $\quad 53$

5 Riemann Hilbert problem $\quad \mathbf{5 8}$

$\begin{array}{lll}5.1 & \text { Introduction } & 58\end{array}$

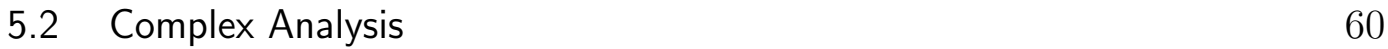

5.3 Cauchy Type integrals 61

5.4 Scalar Riemann-Hilbert Problems $\quad 64$

5.5 Matrix Riemann-Hilbert Problems 68

Diagonal Matrix Riemann Hilbert problems $\quad 69$

Constant Matrix Riemann Hilbert problems $\quad 70$

Rational Matrix Riemann Hilbert Problems $\quad 71$

Triangular Matrix Riemann Hilbert Problems $\quad 73$

5.6 Characterization of Orthogonal Polynomials 74

$\begin{array}{lll}5.7 & \text { Singular Integral Equations } & 75\end{array}$

6 Large asymptotics of orthogonal polynomials: The Riemann-Hilbert $\begin{array}{ll}\text { approach } & \mathbf{7 8}\end{array}$

$\begin{array}{lll}6.1 & \text { Introduction } & 78\end{array}$

6.2 First transformation of the RHP 78

6.3 Second transformation of the RHP 84

6.4 Parametrix at the edge points 93 
6.5 Final transformation and solution of the RHP 102

6.6 Large asymptotics of orthogonal polynomials 105

$\begin{array}{ll}6.7 \text { Concluding remarks } & 106\end{array}$

$\begin{array}{lll}7 & \text { Discussion and Outlook } & 108\end{array}$

$\begin{array}{lll}\text { A Continum limits of Random matrices } & 109\end{array}$

B Properties of the kernel $\quad 111$

C Some considerations of the equivalence between orthogonal polynomials and RHP

$\begin{array}{lr}\text { D Universality } & 122\end{array}$

$\begin{array}{ll}\text { Bibliography } & 124\end{array}$ 


\section{List of Figures}

2.1 Scketch of the resonance spectrum of the Uranium. The qualitative analysis of this spectrum can be approximatly given by eigenvalue statistics of very large random matrices.

2.2 General description of the Gibbs measure that is introduced for the Random Matrix Ensemble later.

2.3 Diagramatic interpretation of the propagator (in the top), where the expectation value of two matrices is given by the coupling of indices with the same direction and the matrix Wick theorem (in the bottom) which consists that the expectation value of a even number of matrices is given by the sum over all the possible couplings.

2.4 Illustration of planar diagrams corresponding to the average $\left\langle\operatorname{Tr} M^{6}\right\rangle$.

2.5 Illustration of the large- $\mathrm{N}$ expansion of matrix integrals corresponding to the topological expansion of the Feymann diagrams.

2.6 Illustration of the correspondence between the quadratic potential of the GUE and Wigner's semicircle law.

2.7 Illustration of a Dyck path for $k=5$. 27

$2.8 \quad$ Typical eigenvalue distribution for $N$ finite. 28

3.1 Pedagogycal illustration of the Dyson gas where one finds the repulsion among the particles and the probability distribution of all particles.

3.2 An illustration of the jump condition for the saddle point equation. 39

4.1 All possibles staircase walks connecting height $n-1$ to height $n$ for $\alpha_{n}^{[2 p+1]}$.

4.2 Staircase walks corresponding to $p=1$. 51

$5.1 \quad$ A general curve for the RHP. $\quad 59$

5.2 An arbitrary oriented curve $\gamma$ for a given jump condition $j_{Y}$. 69

5.3 Representation of the orthogonal polynomial.

6.1 Goal of the first transformation $T_{N}$ : normalize the asymptotic condition of the RHP for the orthogonal polynomials. 80

6.2 Analytic definition(continuation) of $G$. 83

6.3 After the first transformation $T_{N}$ the jump condition was modified. 84

6.4 Analytic continuation of the contours for the jump condition of $T_{N}$. Where quantitatibly the different coulors correspond to different solutions of the RHP for $T_{N}$ such that for large $N$, the yellow and green regions will became only one region.

6.5 After the second transformation $S_{N}$ only the jump condition in the interval $[a, b]$ did not tend to the identity when $N \rightarrow \infty$.

6.6 Problem in the edge point $a$. In this point the jump matrix has a bad behavior by the intersection of four contours. 
6.7 Problem in the edge point $b$. In the same way the jump matrix has a bad behavior by the intersection of four contours $\quad 91$

6.8 Representation of the RHP for M. 92

6.9 Representation of the RHP for $\tilde{M}$. 92

6.10 An illustration of the conformal map $\beta . \quad 96$

6.11 A representation of the Airy parametrix. $\quad 99$

6.12 Analytic continuation of the jump condition after glueing the model RHP to the parametrix.

6.13 After the final transformation $R_{N}$ both jump and asymptotic conditions of the RHP approach to the identity when $N \rightarrow \infty$. 105

6.14 Screenplay of the steepest descent method for solving the RHP that containing information about orthogonal polynomials. 
The devil king of the sixth heaven has roused the ten kinds of troops and, in the midst of the sea of the sufferings of birth and deaths, is at war with the votary of the Lotus Sutra to stop him from taking possession of and to wrest away from him this impure land where both ordinary people and sages dwell.

It has been twenty or more years now since I found myself in that situation and began the great battle. Not once have I thought of retreat.

Nichiren Daishonin

To rise above oneself and to grasp the world.

\section{Archimedes}




\section{1 Introduction}

Random Matrix Theory (RMT) has found different connections between theoretical physics, mathematics and science in general [26]. RMT allows to interplay between these branches. Supplementing and in other cases optimizing some calculations between these researches one can learn more about RMT. A canonical example the use of Random Matrix Theory in applying theoretical physics to mathematics was in number theory. The point is looking for different representations of the same problem to have a wide perspective when solving each problem. In the same spirit the Riemann Hilbert problem applied to RMT allows to obtain different families of continuum theories that one can extend from two-dimensional quantum gravity to number theory. Indeed, the Riemann Hilbert technique provides a method to classify different families of continuum theories by the criticality of the support of the equilibrium measure from RMT.

In this thesis we introduce the theory of unitary ensembles as a particular case of RMT. With respect to this ensemble we interprete its free energy as a topological expansion such that the asymptotic expansion of this energy provides only planar contributions. But the problem is how to compute each topological contribution in the free energy. To overpass this difficulty, we integrate the degrees of freedom of the gauge in the measure, hence we obtain the joint probability distribution function (jpdf) of the eigenvalues which allows to do different calculations, as for example, the partition function. As a special case, we discuss the Gaussian Unitary Ensemble (GUE) and find possible interpretations of the moments of GUE by Catalan numbers and Dyck paths. Furthermore, we show some statistical observables of the eigenvalues that were proved by Riemann Hilbert problems which will be studied in the context of the thesis.

In the third chapter we introduce the Dyson gas as an useful physical analogy that allows to understand the joint probability distribution function (jpdf) of the eigenvalues in thermodynamical equilibrium and in nonequilibrium. A nonequilibrium Dyson gas has a Brownian motion with Smoluchowski equation such that a solution independent of the time is given by the Gibbs measure of the GUE. In the same way all the matrix elements of this ensemble obey 
a Brownian process that for long enough time is given by the Gibbs measure. In the equilibrium case we developed the saddle point equation of the Dyson gas. Furthermore, the resolvent function allows to solve this equation and in consequence find other statistical observables such as the eigenvalue density. In a modern way we rephrased both the saddle point equation and resolvent function as Riemann Hilbert problems. Hence showing the formality of Riemann Hilbert techniques.

Orthogonal polynomials are very especial in Physics and Mathematics. Its vast applications is in part due to the fact that the orthogonal polynomials can be studied under different perspectives. In chapter 4 we formally review the generalities of orthogonal polynomials. In the following we study the orthogonal polynomials from different points of view. From a combinatorial point of view the orthogonal polynomials can be represented as three term recurrence relations. Analytically, the orthogonal polynomials are represented by a string equation which is shows below. We introduce the relation between a string equation and the staircase path. In this way the orthogonal polynomials can be represented geometrically as staircase paths. Algebraically we represent orthogonal polynomials by commutation relation. Statistically the orthogonal polynomials are represented by a kernel and hence we compute the $n$-point correlation function of the eigenvalues in function of orthogonal polynomials. Furthermore, orthogonal polynomial can be represented as Riemann Hilbert problem that will be studied in the next chapter.

The Riemann Hilbert problem (RHP) is a powerfull method in asymptotic analysis of differential and integral equations with applications in many areas, in particular orthogonal polynomials and Random Matrix Theory(RMT). Also we introduce basic notions of complex analysis such as the theory of Cauchy type integrals. We discuss the scalar RHP. This discussion is generalized to the matrix RHP such that in some cases matrix RHP can be reduced to the analysis of an array of scalar RHPs. When this is not possible the analysis is given by singular integral equations. Furthermore we review the relation of orthogonal polynomials in RMT and RHPs.

Many statistical quantities of Hermitian Random Matrices can be reduced to calculations involving large asymptotics of orthogonal polynomials. Finally we present the Riemann-Hilbert approach to the large asymptotics of orthogonal polynomials. This approach is based on the steepest descent method, which provides an asymptotic solution to the RHP, as $N \rightarrow \infty$. 


\section{2}

\section{Random Matrix Theory}

We can find applications of Random Matrix Theory at theoretical physics, mathematics and science in general on the book [26]. A few examples of emergence of Random Matrix Theory range from two-dimensional quantum gravity [3], towards biological models [4] and number theory [5]. In this chapter we introduce the unitary ensemble of random matrices. Fixing the gauge of the ensemble, we obtain the joint probability distribution function (jpdf) of the eigenvalues. In particular, we discuss the Gaussian unitary ensemble (GUE) and possible interpretations of the moments of GUE. Finally we present some statistical properties of the eigenvalues that will be proved in a further chapter by Riemann Hilbert problems. In the third section of this chapter we follow the exposure of [11], whereas the fourth section is based on the text [29]. For more detail the reader should consult [31].

\section{1 \\ Introduction}

RMT originated with the works of Hsu, Wishart, and others, in mathematical statistics around 1920s [1]. In the 1950s the theory had its first steps in physics with the work of Wigner as an attempt to describe the statistical fluctuations of random operators in many body quantum systems. In fact, Wigner suggested resonance spectra of heavy nuclei can be described in terms of the eigenvalues of very large matrices. Just like any resonant system can be characterized by a Hamiltonian, Wigner considered that eigenvalue statistics of this Hamiltonian was only restricted to symmetries of this Hamiltonian. Using this fact he found that the average density of eivenvalues for real symmetric matrices is given by the semicircle law in the limit of large matrix size. Next, more detailed work on other observables and different ensembles, was studied by Dyson and Metha. While later, the method of orthogonal polynomials was developed for the GUE by Metha and Gaudin.

Furthermore, a relation between RMT and two-dimensional quantum gravity was found. This relation began with the work of t'Hooft [28], where the expansion of the free energy in terms of Feymann diagrams for a field theory with large gauge group is dominated by planar Feymann diagrams. This 


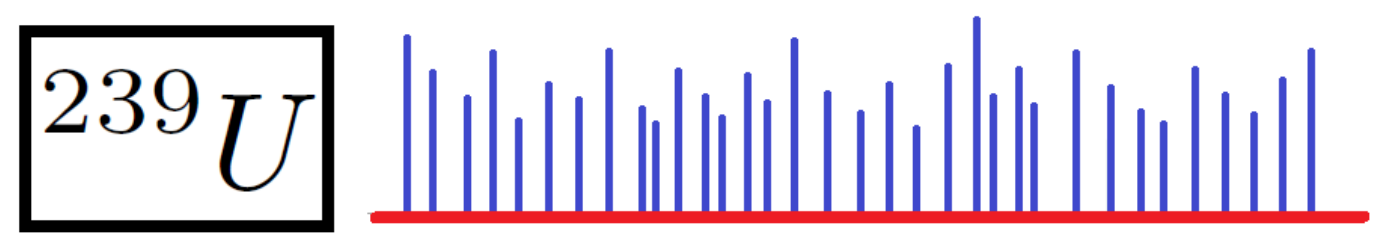

Figure 2.1: Scketch of the resonance spectrum of the Uranium. The qualitative analysis of this spectrum can be approximatly given by eigenvalue statistics of very large random matrices.

expansion was introduced in RMT by Brézin, Itzykson, Parisi and Zuber [6] such that random matrix integrals using an appropriate limit scaling generate planar graphs. Further developments showed that a more exact method for finding statistical properties of eigenvalues was the orthogonal polynomial method. Later, orthogonal polynomials were rephrased as Riemann Hilbert problems [14]. Hence, Riemann Hilbert problems turn out to be a powerfull method in RMT.

All these reflect the wide variety of resources in RMT.

Outline: The chapter is organized as follows. Section 2.2 contains basic notions of probability space and of ensembles. Section 2.3 gives a review of unitary ensembles and its topological large $N$ expansion. In section 2.4 we introduce the ensemble of eigenvalues. In section 2.5 we describe the Gaussian Unitary Ensemble. Following by the statistical obsevables that were proved with Riemann Hilbert problems.

\section{2}

\section{Statistical ensemble}

Observing that entries of random matrices are random variables in a probability space, we will review some topics of random variable and statistical ensembles. Recall that probability space of random variables is a measure space such that the measure of the whole space is equal to one. In general, this space is a mathematical construction that models a real process and consists of three parts:

1. Sample space $\Omega$, which is a set of all possible outcomes. Where the outcomes may be states of nature, possibilities, experimental results among others. Since individual outcomes could be of little practical use, then more complex events are used to characterize groups of outcomes. A collection of all such events corresponds to a $\sigma$-algebra $\mathcal{F}$.

2. $\sigma$-algebra $\mathcal{F}$, which is a collection of all the subsets of the sample space that we would like to consider. 
3. Probability measure $P$, which is given by the assignment of probabilities to the events. Where probability is a real number between zero (event never happens) and one (event happens with total certainty). Indeed, a function $P: \mathcal{F} \rightarrow[0,1]$ is a measure on $\mathcal{F}$ if it is countably additive and $P(\Sigma)=1$.

In this framework, the probability space of $N \times N$ random matrices is given by $(\Omega, \mathcal{F}, P)$, so that a random matrix $M$ is a measurable map from $(\Omega, \mathcal{F})$ to $M_{N}(\mathrm{C})$, where $M_{N}(\mathrm{C})$ denotes the space of $N \times N$ matrices with entries in the complex plane $\mathrm{C}$. The measurable map is determined by the invariants of the matrix such as $\mathrm{E}\left(\operatorname{Tr} M^{k}\right)$, where $\mathrm{E}$ is the expectation with respect to $P$ and $\operatorname{Tr}$ is the trace on $M_{N}(\mathrm{C})$.

The choice of $P$ is promoted by thermodynamic ensembles. A thermodynamic ensemble is a statistical ensemble that is in equilibrium and can be isolated, closed or open. This ensembles are specified by

1. Microcanonical ensemble, is a set of microstates such that when a macroscopic system is in thermodynamic equilibrium and this system is isolate, all the microstates have approximately the same role in the definition of the macrostate. Thus a probability of finding one system with energy $E_{r}$ is given by

$$
P\left(E_{r}\right)=\left\{\begin{array}{cc}
\frac{1}{\Omega(E)} & E=E_{r} \\
0 & \text { otherwise }
\end{array},\right.
$$

where $\Omega(E)$ is a microcanonical partition function. This partition function gives a number of available states for the energy $E_{r}$.

2. Canonical ensemble, is associated with a system that can exchange energy with a reservoir. So the probability to find a system with energy $E_{r}$ is given by

$$
P\left(E_{r}\right)=\frac{e^{-\beta E_{r}}}{Z(\beta)}
$$

which is known as Gibbs measure, where $Z(\beta)$ is the canonical partition function,

$$
Z(\beta)=\sum_{r} e^{-\beta E_{r}}
$$

and $e^{-\beta E_{r}}$ is called Boltzmann weight.

It is important to realize that the canonical ensemble is mathematically equivalent to the microcanonical ensemble, in the sense that although the canonical ensemble contains systems of all energies, the majority of them have the same energy in the microcanonical esemble. Indeed one 


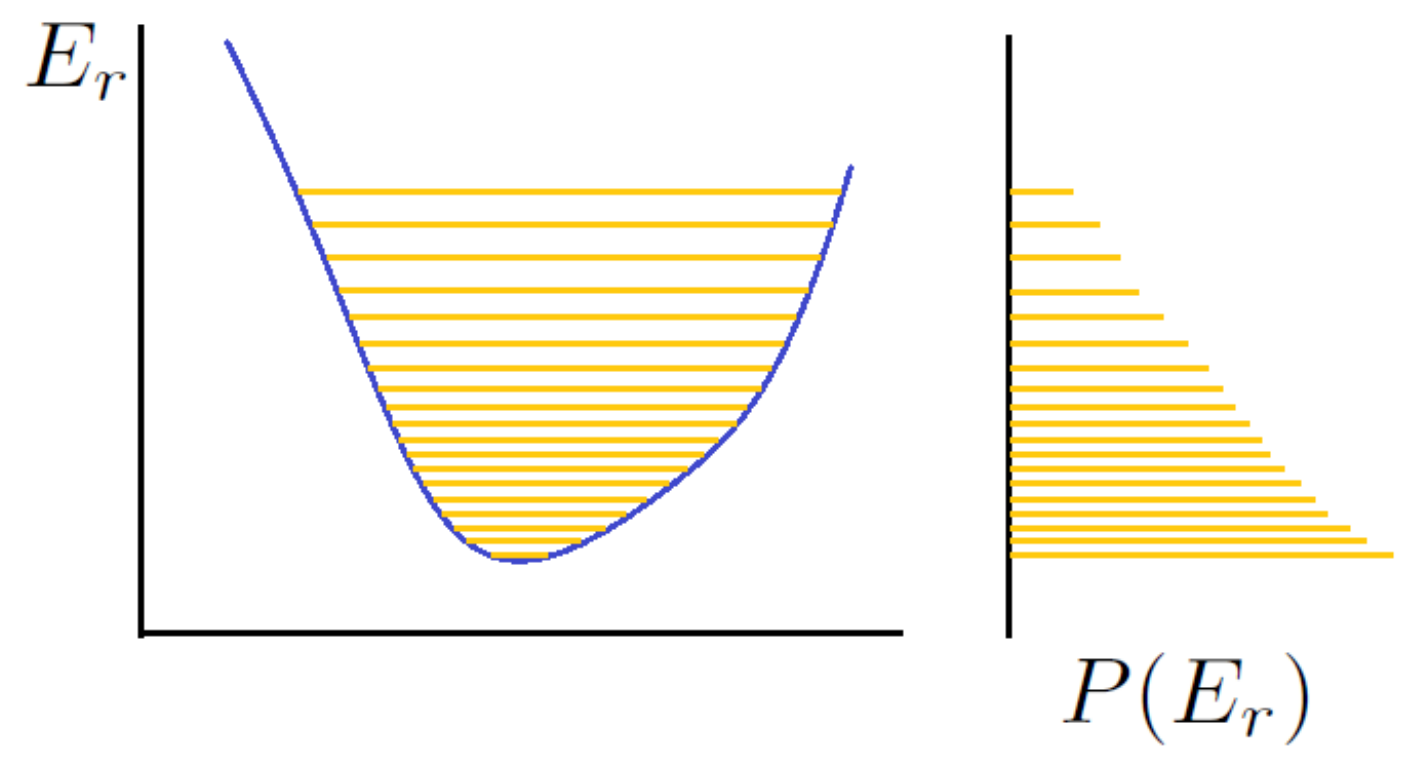

Figure 2.2: General description of the Gibbs measure that is introduced for the Random Matrix Ensemble later.

can fix $E_{r}=E$ to obtain

$$
\begin{aligned}
z(\beta) & =\sum_{E} \sum_{r, E_{r}=E} e^{-\beta E_{r}} \\
& =\sum_{E} e^{-\beta E} \sum_{i, E_{i}=E} 1 \\
& =\sum_{E} e^{-\beta E} \Omega(E)
\end{aligned}
$$

implying that the canonical partition function corresponds to the generating function of the microcanonical partition function.

3. Grand canonical ensemble, corresponds to a system such that if it exchanges particles with the reservoir too, then the Grand canonical partition function is the generating function of the canonical partition function

$$
\Theta(\beta, z)=\sum_{N} Z_{N}(\beta)\left(e^{\beta \mu}\right)^{N}
$$

where $e^{\beta \mu}$ is the fugacity of the chemical potential $\mu$ which is the probability of expansion of the particle. For this reason one can obtain the canonical partition function by inverse Laplace transform,

$$
Z_{N}(\beta)=\frac{1}{2 \pi i} \oint d z \frac{\Theta(\beta, z)}{z^{N+1}}
$$




\section{3}

\section{Unitary ensemble of random matrices}

The Unitary ensemble for random matrices is given by a measure $d \mu_{N}(M)$, so that this measure is invariant under global transformations $U(N)$. The matrix elements $M_{i j}$ are complex valued random variables which are taken from a probability distribution $d \mu_{N}(M)$ of the $\left(\Omega, \mathcal{F}, d \mu_{N}(M)\right)$. In fact, given a set of $N \times N$ random Hermitian matrices

$$
M=\left(\begin{array}{cccc}
M_{11} & \Re M_{12}+i \Im M_{12} & \ldots & \Re M_{1 N}+i \Im M_{1 N} \\
\Re M_{12}-i \Im M_{12} & M_{22} & \ldots & \Re M_{2 N}+i \Im M_{2 N} \\
\vdots & \vdots & \ddots & \vdots \\
\Re M_{1 N}-i \Im M_{1 N} & \Re M_{2 N}-i \Im M_{2 N} & \ldots & M_{N N}
\end{array}\right)
$$

we define a Gibbs measure for Hermitian matrices $M$, in the following way

$$
d \mu_{N}(M)=\frac{1}{Z_{N}^{\prime}} e^{-N T r V(M)} d M,
$$

where $Z_{N}^{\prime}=\int e^{-N T r V(M)} d M$, is the partition function and $d M$ corresponds to the Lebesgue mesure:

$$
d M=\Pi_{j=1}^{N} d M_{j j} \Pi_{j \neq k} d \Re M_{j k} d \Im M_{j k},
$$

notice that we assumed independence between the matrix elements initially, but the so-called potential $V$ correlates the matrix elements subsequently. Since the trace is invariant under unitary transformations, $U U^{\dagger}=U^{\dagger} U=I$, we have

$$
\operatorname{Tr} V(M)=\operatorname{Tr} V\left(U^{\dagger} M^{\prime} U\right)=\operatorname{Tr} V\left(M^{\prime} U U^{\dagger}\right)=\operatorname{Tr} V\left(M^{\prime}\right) .
$$

Therefore the Gibbs measure is invariant under unitary transformations, $M \rightarrow$ $U^{\dagger} M^{\prime} U, U \in U(N)$.

As an illustration, for $V(M)=\frac{1}{2} M^{2}$, the expectation value of $f(M)$ in the Gaussian Unitary Ensemble, is given by

$$
\langle f(M)\rangle=\frac{\int f(M) e^{-\frac{N}{2} T r M^{2}}}{\int e^{-\frac{N}{2} T r M^{2}}} .
$$

Using the so-called source integral method [21] in this ensemble, we have

$$
\left\langle M_{i j} M_{k l}\right\rangle=\frac{\delta_{i l} \delta_{j k}}{N},
$$

This source integral method can be generalized to the matrix Wick theorem

$$
\left\langle\prod_{(i, j) \in I} M_{i j}\right\rangle=\sum_{\text {pairings }} P \prod_{(i j),(k l) \in P}\left\langle M_{i j} M_{k l}\right\rangle .
$$


Now suppose that $V(M)$ be a formal power series in the $g_{i}$, of the following

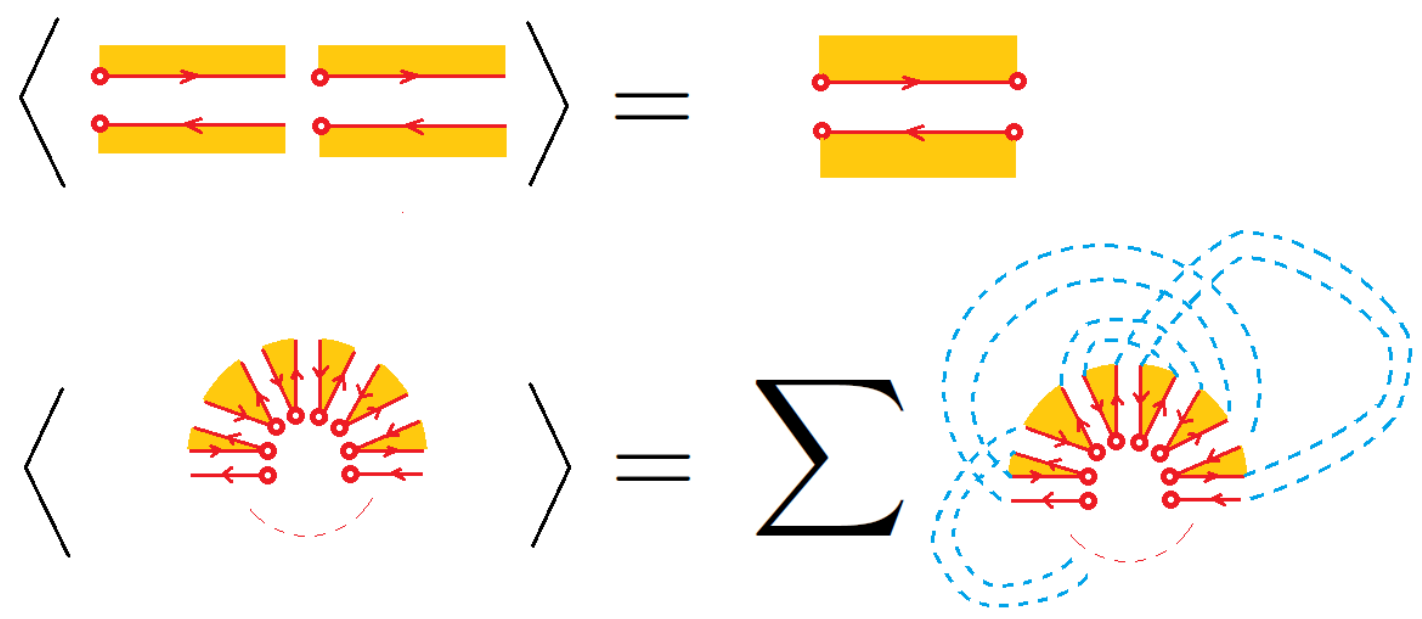

Figure 2.3: Diagramatic interpretation of the propagator (in the top), where the expectation value of two matrices is given by the coupling of indices with the same direction and the matrix Wick theorem (in the bottom) which consists that the expectation value of a even number of matrices is given by the sum over all the possible couplings.

form

$$
V(M)=\sum_{i \geq 3} \frac{g_{i}}{i} \operatorname{Tr} M^{i}
$$

hence the partition function can be expressed as a expectation value,

$$
Z_{N}\left(g_{3}, g_{4}, \ldots\right)=\left\langle e^{-N \sum_{i \geq 3} \frac{g_{i}}{i} T r M^{i}}\right\rangle,
$$

using the Taylor expansion of the exponential yields

$$
Z_{N}\left(g_{3}, g_{4}, \ldots\right)=\sum_{n_{1}, n_{2}, \ldots} \prod_{i \geq 3} \frac{\left(-N g_{i}\right)^{n_{i}}}{i^{n_{i}} n_{i} !}\left\langle\prod_{i \geq 3} \operatorname{Tr}\left(M^{i}\right)^{n_{i}}\right\rangle .
$$

Since the expectation value can be rephrased as a sequence of $n_{i}$ i-valent vertices, connecting the oriented double links into pairs forms a closed graph $\Gamma$ with double lines. By the matrix Wick theorem, we obtain

$$
Z_{N}\left(g_{3}, g_{4}, \ldots\right)=\sum_{n_{1}, n_{2}, \ldots} \prod_{i \geq 3} \frac{\left(-N g_{i}\right)^{n_{i}}}{i^{n_{i}} n_{i} !} \quad \sum_{\text {all labelled } \Gamma} \quad N^{-E(\Gamma)} N^{F(\Gamma)},
$$

with $n_{i} i$-valent vertices

where $E(\Gamma)$ is the number of edges of $\Gamma$ and $F(\Gamma)$ is the number of faces of $\Gamma$. Summing over all the possible labelings of an unlabeled graph $\Gamma$, we find [11]

$$
Z_{N}\left(g_{3}, g_{4}, \ldots\right)=\sum_{\text {all } \Gamma} \frac{N^{V(\Gamma)-E(\Gamma)+F(\Gamma)}}{|A u t \Gamma|} \prod_{i}\left(-g_{i}\right)^{n_{i}(\Gamma)},
$$



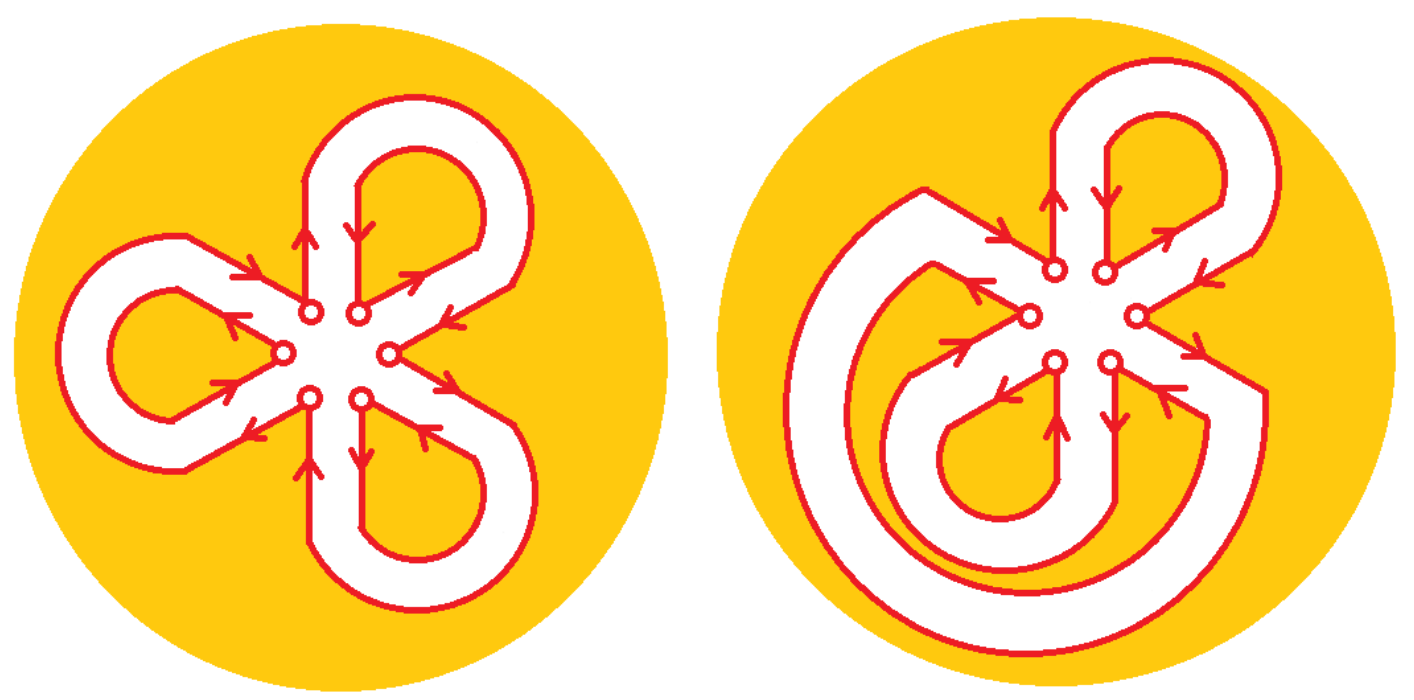

Figure 2.4: Illustration of planar diagrams corresponding to the average $\left\langle\operatorname{Tr} M^{6}\right\rangle$.

where $n_{i}(\Gamma)$ is the total number of $i$-valent vertices of $\Gamma,|A u t \Gamma|$ is the symmetry group of $\Gamma$ which cancels the repetitions of the graph on the equivalent topological surfaces and $V(\Gamma)$ is the number of vertices of $\Gamma$. Since the number $V(\Gamma)-E(\Gamma)+F(\Gamma)$ characterizes the topology of the surface where the graph is drawn, we obtain

$$
Z_{N}\left(g_{3}, g_{4}, \ldots\right)=\sum_{\text {all } \Gamma} \frac{N^{2-2 g}}{|A u t \Gamma|} \prod_{i}\left(-g_{i}\right)^{n_{i}(\Gamma)},
$$

where $2-2 g=V(\Gamma)-E(\Gamma)+F(\Gamma)=\chi(\Gamma)$ is called Euler-Poincare characteristic of the dual of $\Gamma$ which is a tessellation $\Gamma^{*}$ of a Riemann surface. So that $g$ is the number of holes of this Riemann surface.

Hence a normalized free energy is given by taking the logarithm to $Z_{N}$, restricted only to connected graphs [31]

$$
F_{N}^{0}\left(g_{3}, g_{4}, \ldots\right)=\log Z_{N}\left(g_{3}, g_{4}, \ldots\right)
$$

because, in the Taylor expansion of $\log F_{N}$ the contribution of the disconnected graphs are cancelled between terms of this expansion. Then, we get

$$
F_{N}^{0}\left(g_{3}, g_{4}, \ldots\right)=\sum_{\text {connected } \Gamma} \frac{N^{2-2 g}}{|A u t \Gamma|} \prod_{i}\left(-g_{i}\right)^{n_{i}(\Gamma)} .
$$

As $g$ is a topological invariant,

$$
F_{N}^{0}\left(g_{3}, g_{4}, \ldots\right)=\sum_{\text {connected } \Gamma} N^{2-2 g} F^{g}\left(g_{3}, g_{4}, \ldots\right),
$$

is called the topological large $N$ expansion. 


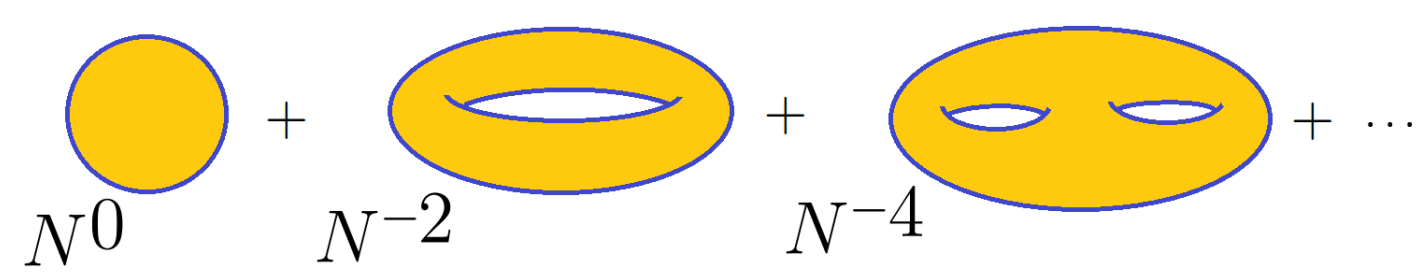

Figure 2.5: Illustration of the large-N expansion of matrix integrals corresponding to the topological expansion of the Feymann diagrams.

\section{4}

\section{Ensemble of eigenvalues}

By gauge symmetry one can reduce the number of parameters of the Gibbs measure from $N^{2}$ to $N$, thence expressing this measure only as function of the eigenvalues of $M$ by the gauge fixing [29]. In the following we will show how this gauge fixing works by using a more physical approach given by the references [29][27].

Recall that in general, the Dirac delta function satisfies

$$
\int d g(x) \delta(g(x))=1
$$

which can be written in the following way

$$
\int d g(x) \delta(g(x))=\int d x\left|\frac{d g}{d x}\right| \delta(g(x))=1 .
$$

Analogously, for the matricial functions we can define a matrix distribution $\delta$ before fixing the gauge. For convenience if one fixes the gauge at $F=0$, we obtain

$$
\int d U\left|\frac{\delta F\left({ }^{U} M\right)}{\delta U}\right|_{F=0} \delta\left(F\left({ }^{U} M\right)\right)=1,
$$

where $\delta F\left({ }^{U} M\right)=\prod_{i<j} \delta^{(2)}\left(F_{i j}\left({ }^{U} M\right)\right)$ and ${ }^{U} M=U^{\dagger} M U$. Using the gauge invariance we have

$$
\left|\frac{\delta F\left({ }^{U} M\right)}{\delta U}\right|_{F=0}=\left|\frac{\delta F(M)}{\delta U}\right|_{F=0},
$$

thus, we insert this equality into the integral (2.15) to get

$$
\left|\frac{\delta F(M)}{\delta U}\right|_{F=0} \int d U \delta\left(F\left({ }^{U} M\right)\right)=1 .
$$

If one consideres $M$ as an almost diagonal matrix, then $U$ is approximately the identity matrix, because we do not need to change $M$ significantly. Therefore,

$$
U \cong I+A
$$


since the matrix $A$ has small entries, then $U \cong e^{A}$. For this reason one can expres the measure $d U$ as functions of the matrix elements of $A$, in the following way

$$
d U=d u \prod_{i<j} d\left(\Re A_{i j}\right) d\left(\Im A_{i j}\right)
$$

where $d u$ is the measure over the diagonal of $U$, such that in particular $\int d u=1$. Therefore the integral (2.16) can be expressed as

$$
\left|\frac{\delta F(M)}{\delta U}\right|_{F=0} \int d u \prod_{i<j} d\left(\Re A_{i j}\right) d\left(\Im A_{i j}\right) \delta\left(F\left({ }^{U} M\right)\right)=1
$$

As the gauge is given by, $F_{i j}\left({ }^{U} M\right)=\left(U^{\dagger} M U\right)_{i j}, i \neq j$, and this gauge does not depend on $u$, we have

$$
\left(\int d u\right)\left|\frac{\delta F(M)}{\delta U}\right|_{F=0} \int \prod_{i<j} d\left(\Re A_{i j}\right) d\left(\Im A_{i j}\right) \delta^{2}\left(U^{\dagger} M U\right)_{i j}=1
$$

Now we will obtain the constraints on $M$ by gauge fixing, $\left(U^{\dagger} M U\right)_{i j}=0, i \neq j$, hence we have

$$
\left(U^{\dagger} M U\right)_{i j}=\sum_{k l}\left(e^{-A}\right)_{i k} M_{k l}\left(e^{A}\right)_{l j}=0, i \neq j
$$

also since $M_{l l} \neq 0$, and $e^{A} \cong I+A$, we can write the gauge fixing as

$$
\begin{aligned}
\sum_{l}(I-A)_{i l} M_{l l}(I+A)_{l j} & =0, i \neq j \\
\sum_{l}\left(I_{i j}-A_{i l} I_{l j}+I_{i l} A_{l j}-A_{i l} A_{l j}\right) M_{l l} & =0 \\
\sum_{l}\left(-A_{i l} A_{l j}\right) M_{l l} & =0
\end{aligned}
$$

thus for $i \neq j$ we get

$$
\sum_{l} A_{i l} A_{l j}=A_{i j}^{2}=0 .
$$

In conclusion, the gauge fixing is equivalent to

$$
A_{i j}=0, \quad i \neq j
$$


Furthermore, we will use this condition to reduce the integral (2.17). Using $\int d u=1$ and $U=I+A$ this integral can be espressed as

$$
\begin{aligned}
\left|\frac{\delta F(M)}{\delta U}\right|_{F=0} \int \prod_{i<j} d\left(\Re A_{i j}\right) d\left(\Im A_{i j}\right) \delta^{2}((I-A) M(I+A))_{i j} & =1 \\
\left|\frac{\delta F(M)}{\delta U}\right|_{F=0} \int \prod_{i<j} d\left(\Re A_{i j}\right) d\left(\Im A_{i j}\right) \delta^{2}(M A-A M-A M A+I)_{i j} & =1
\end{aligned}
$$

as can be noted $M_{i j} \cong \lambda_{i} \delta_{i j}$, then we can rewrite this integral as

$$
\begin{aligned}
\frac{1}{\left|\frac{\delta F(M)}{\delta U}\right|_{F=0}}= & \int \prod_{i<j} d\left(\Re A_{i j}\right) d\left(\Im A_{i j}\right) \times \\
& \times \delta^{2}\left(\sum_{l} \lambda_{i} \delta_{i l} A_{l j}-\sum_{m} A_{i m} \lambda_{m} \delta_{m j}-\sum_{n r} A_{i n} \lambda_{n} \delta_{n r} A_{r j}+I_{i j}\right)
\end{aligned}
$$

by the property of the $\delta$ distribution, we obtain

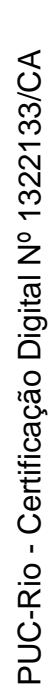

$$
\begin{aligned}
\frac{1}{\left|\frac{\delta F(M)}{\delta U}\right|_{F=0}=} & \int \prod_{i<j} d\left(\Re A_{i j}^{\prime}\right) d\left(\Im A_{i j}^{\prime}\right) \times \\
& \frac{\prod_{i<j} \delta\left[\Re\left(A_{i j}-A_{i j}^{\prime}\right) \Im\left(A_{i j}-A_{i j}^{\prime}\right)\right]}{\prod_{i<j}\left(\lambda_{j}-\lambda_{i}-\sum_{r} A_{i r}^{\prime} \lambda_{r} \delta_{r j}\right) \prod_{i<j}\left(\lambda_{j}-\lambda_{i}-\sum_{r} A_{i r}^{\prime} \lambda_{r} \delta_{r j}\right)}
\end{aligned}
$$

Finally, using the gauge fixing (2.18) we find

$$
\left|\frac{\delta F(M)}{\delta U}\right|_{F=0}=\prod_{i<j}\left(\lambda_{j}-\lambda_{i}\right)^{2}
$$

Consequently, the partition function is given by

$$
\begin{aligned}
Z_{N}^{\prime} & =\int d M e^{-N T r V(M)}\left|\frac{\delta F(M)}{\delta U}\right|_{F=0} \int d U \delta\left(F\left({ }^{U} M\right)\right) \\
& =\int d U d M e^{-N T r V(M)} \prod_{i<j}\left(\lambda_{j}-\lambda_{i}\right)^{2} \delta\left(F\left({ }^{U} M\right)\right) \\
Z_{N}^{\prime} & =\int d U \int \prod_{i=1}^{N} d \lambda_{i} e^{-N V\left(\lambda_{i}\right)} \prod_{i<j}\left(\lambda_{i}-\lambda_{j}\right)^{2}
\end{aligned}
$$

So if one defines $Z_{N}=\frac{Z_{N}^{\prime}}{\int d U}$ we obtain

$$
Z_{N}=\int \prod_{i=1}^{N} d \lambda_{i} e^{-N V\left(\lambda_{i}\right)} \prod_{i<j}\left(\lambda_{i}-\lambda_{j}\right)^{2}
$$

In essence, by integrating out $U$ one obtains the $j p d f$ of the eigenvalues 


$$
d \mu_{N}(\lambda)=\frac{1}{Z_{N}} \prod_{i<j}\left(\lambda_{i}-\lambda_{j}\right)^{2} \prod_{i=1}^{N} e^{-N V\left(\lambda_{i}\right)} d \lambda_{i},
$$

where $Z_{N}=\int \prod_{i<j}\left(\lambda_{i}-\lambda_{j}\right)^{2} \prod_{i=1}^{N} e^{-N V\left(\lambda_{i}\right)} d \lambda_{i}$ is the partition function. This $j p d f$ allows to intrepete and calculate the correlation between eigenvalues. One can observe that the eigenvalues are dependent random variables due to the factor $\prod_{i<j}\left(\lambda_{i}-\lambda_{j}\right)^{2}$ which is called Van der Monde determinant.

\section{5}

\section{Gaussian unitary ensemble}

A special case of unitary emsembles is given when $V(M)=\frac{1}{2} M^{2}$ which corresponds to the Gaussian Unitary Ensemble. For this case, we write the partition function of the GUE as

$$
Z_{N}^{\prime G U E}=\int e^{\frac{N}{2} \operatorname{Tr} M^{2}} d M
$$

In this case, we express the trace in terms of the matrix elements,

$$
\operatorname{Tr} M^{2}=\sum_{i, j} M_{i j} M_{j i}=\sum_{i, j}\left(\Re M_{i j}+i \Im M_{i j}\right)\left(\Re M_{j i}+i \Im M_{j i}\right)
$$

by the hermiticity of $M$, we have

$$
\begin{aligned}
\operatorname{Tr} M^{2} & =\sum_{i, j}\left(\Re M_{i j}\right)^{2}+\left(\Im M_{i j}\right)^{2} \\
& =\sum_{i} M_{i i}^{2}+2 \sum_{i<j}\left(\Re M_{i j}\right)^{2}+2 \sum_{i<j}\left(\Im M_{i j}\right)^{2} \\
\operatorname{Tr} M^{2} & =\sum_{i} M_{i i}^{2}+2 \sum_{i<j}\left|M_{i j}\right|^{2}
\end{aligned}
$$

hence the probability distribution of GUE is given by

$$
d \mu_{N}^{G U E}(M)=\frac{1}{Z_{N}^{\prime G U E}} \Pi_{i}\left(e^{-\frac{N}{2} M_{i i}^{2}}\right) \Pi_{i<j}\left(e^{N\left|M_{i j}\right|^{2}}\right) d M
$$

Here one observes that the matrix elements in GUE are independent Gaussian random variables. Therefore integrating each random variable, we obtain the partition function of GUE,

$$
Z_{N}^{\prime G U E}=\int \Pi_{i}\left(e^{-\frac{N}{2} M_{i i}^{2}}\right) \Pi_{i<j}\left(e^{N\left|M_{i j}\right|^{2}}\right) d M=2^{\frac{N^{2}-N}{2}}\left(\frac{\pi}{N}\right)^{\frac{N^{2}}{2}} .
$$

On the other hand, the $j p d f$ of the GUE is given by

$$
d \mu_{N}^{G U E}(\lambda)=\frac{1}{Z_{N}^{G U E}} \Pi_{i<j}\left(\lambda_{i}-\lambda_{j}\right)^{2} \Pi_{i=1}^{N} e^{-\frac{N}{2} \lambda_{i}} d \lambda_{i}
$$


where $Z_{N}^{G U E}$ corresponds to a Selberg integral, so that

$$
Z_{N}^{G U E}=\int \Pi_{i<j}\left(\lambda_{i}-\lambda_{j}\right)^{2} \Pi_{i=1}^{N} e^{-\frac{N}{2} \lambda_{i}} d \lambda_{i}=\frac{(2 \pi)^{\frac{N}{2}}}{N^{\frac{N^{2}}{2}}} \Pi_{n=1}^{N} n ! .
$$

Notice that the ratio between $Z_{N}^{\prime G U E}$ and $Z_{N}^{G U E}$ gives us the volume $\int d U$. In addition, given a finite $N$ and $\lambda$, we define the eigenvalue distribution as the normalized random probability measure on the real line $\mathrm{R}$ given by

$$
\rho_{N}(\lambda)=\frac{1}{N} \sum_{i=1}^{N}\left\langle\delta\left(\lambda-\lambda_{i}\right)\right\rangle
$$

and for the large $N$ limit this distribution becomes an eigenvalue density

$$
\rho(\lambda)=\lim _{N \rightarrow \infty} \rho_{N}(\lambda)=\frac{1}{2 \pi} \sqrt{4-\lambda^{2}}
$$

which is the famous Wigner's semi-circle law.

We also define the moments of the eigenvalue density as

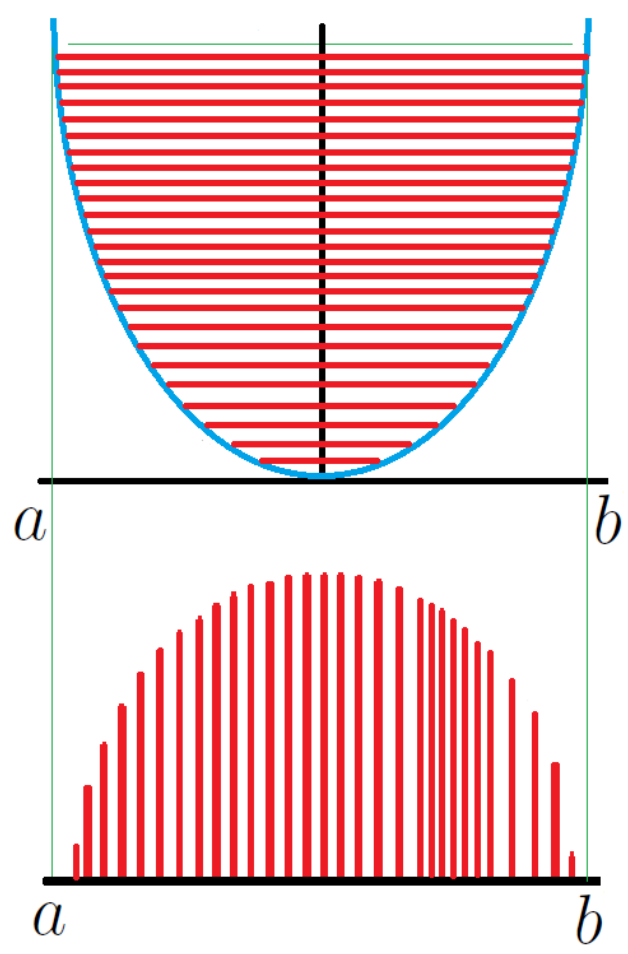

Figure 2.6: Illustration of the correspondence between the quadratic potential of the GUE and Wigner's semicircle law.

$$
m_{k}=\int \lambda^{k} \rho(\lambda) d \lambda
$$


If we write $\lambda=2 \sin \theta$, then $\rho(\lambda)=\frac{1}{\pi} \cos \theta$ and $d \lambda=2 \cos \theta d \theta$, the moment $m_{2 k}$ is given by

$$
\begin{aligned}
m_{2 k} & =\int_{-2}^{2} \lambda^{2 k} \rho(\lambda) d \lambda=\int_{-\frac{\pi}{2}}^{\frac{\pi}{2}}(2 \sin \theta)^{2 k}\left(\frac{1}{\pi} \cos \theta\right)(2 \cos \theta d \theta) \\
& =\frac{2^{2 k+1}}{\pi} \int_{-\frac{\pi}{2}}^{\frac{\pi}{2}} \sin ^{2 k} \theta \cos ^{2} \theta d \theta \\
& =\frac{2^{2 k+1}}{\pi}\left(\int_{-\frac{\pi}{2}}^{\frac{\pi}{2}} \sin ^{2 k} \theta d \theta-(2 k+1) \int_{-\frac{\pi}{2}}^{\frac{\pi}{2}} \sin ^{2 k} \theta \cos ^{2} \theta d \theta\right) \\
m_{2 k} & =\frac{2^{2 k+1}}{\pi} \int_{-\frac{\pi}{2}}^{\frac{\pi}{2}} \sin ^{2 k} \theta d \theta-(2 k+1) m_{2 k} .
\end{aligned}
$$

which simplifies to

$$
\begin{aligned}
m_{2 k} & =\frac{2^{2 k+1}}{\pi(2 k+2)} \int_{-\frac{\pi}{2}}^{\frac{\pi}{2}} \sin ^{2 k} \theta d \theta \\
& =\frac{2^{2 k+1}(2 k-1)}{\pi(2 k+2)} \int_{-\frac{\pi}{2}}^{\frac{\pi}{2}} \sin ^{2 k-2} \theta \cos ^{2} \theta d \theta \\
m_{2 k} & =\frac{2^{2}(2 k-1)}{2 k+2} m_{2 k-2} .
\end{aligned}
$$

To solve this recursion relation, we compute $m_{0}$ in the following way,

$$
m_{0}=\frac{2}{\pi} \int_{-\frac{\pi}{2}}^{\frac{\pi}{2}} \cos ^{2} \theta d \theta=1 .
$$

Therefore, the moment $m_{2 k}$ is given by

$$
\begin{aligned}
m_{2 k} & =\frac{2^{2}(2 k-1)}{2 k+2} \frac{2^{2}(2 k-3)}{2 k} \frac{2^{2}(2 k-5)}{2 k-2} \ldots \frac{2^{2}}{4} \\
& =\frac{(2 k) !}{(k+1) ! k !} \\
& =\frac{1}{k+1}\left(\begin{array}{c}
2 k \\
k
\end{array}\right) \\
m_{2 k} & =C_{k}
\end{aligned}
$$

which is known as Catalan number. The moments $m_{2 k+1}$ read

$$
m_{2 k+1}=\frac{2^{2 k+2}}{\pi} \int_{-\frac{\pi}{2}}^{\frac{\pi}{2}} \sin ^{2 k+1} \theta \cos ^{2} \theta d \theta,
$$

but the integral of an odd function over a symmetric interval is zero

$$
m_{2 k+1}=0 \text {. }
$$


Actually, the Catalan numbers $C_{k}$ possess many interpretations. For instance, we consider a Bernoulli walk of length $l$ which is a sequence $\left\{s_{n}\right\}_{0 \leq n \leq l} \subset \mathrm{Z}$ such that $s_{0}=0$ and $\left|s_{t+1}-s_{t}\right|=1$ for $t \leq l-1$. In fact, $C_{k}$ counts the number of nonnegative Bernoulli walks of length $2 k$ that terminate at 0 . These walks are know as Dyck paths.

Indeed, let $D_{k}$ corresponds to the number of Dyck paths. If $B_{k}$ denotes the number of Bernoulli walks $\left\{s_{n}\right\}$ of length $2 k$ that only satisfy $s_{2 k}=0$, and $\tilde{B}_{k}$ denotes the number of Bernoulli walks $\left\{s_{n}\right\}$ of length $2 k$ that satisfy $s_{2 k}=0$ and $s_{t}<0$ for some $t<2 k$, then, $D_{k}=B_{k}-\tilde{B}_{k}$. Now $B_{k}$ consists of $k$ jumps up and $k$ jumps down, so $B_{k}=\left(\begin{array}{c}2 k \\ k\end{array}\right)$. Next $\tilde{B}_{k}$ equals to the number of Bernoulli walks starting at 0 and ending at -2 , because if one consideres $t$ the last visit to -1 , and by reflection of the walk after $t$ at -1 , one obtains a unique walk ending at -2 with the same set of visits to -1 . Therefore, $\tilde{B}_{k}$ is given by $k-1$ jumps up and $k+1$ jumps down, so $\tilde{B}_{k}=\left(\begin{array}{c}2 k \\ k-1\end{array}\right)$. Thus we conclude that

$$
D_{k}=B_{k}-\tilde{B}_{k}=\left(\begin{array}{c}
2 k \\
k
\end{array}\right)-\left(\begin{array}{c}
2 k \\
k-1
\end{array}\right)=\frac{1}{k+1}\left(\begin{array}{c}
2 k \\
k
\end{array}\right)=C_{k}
$$

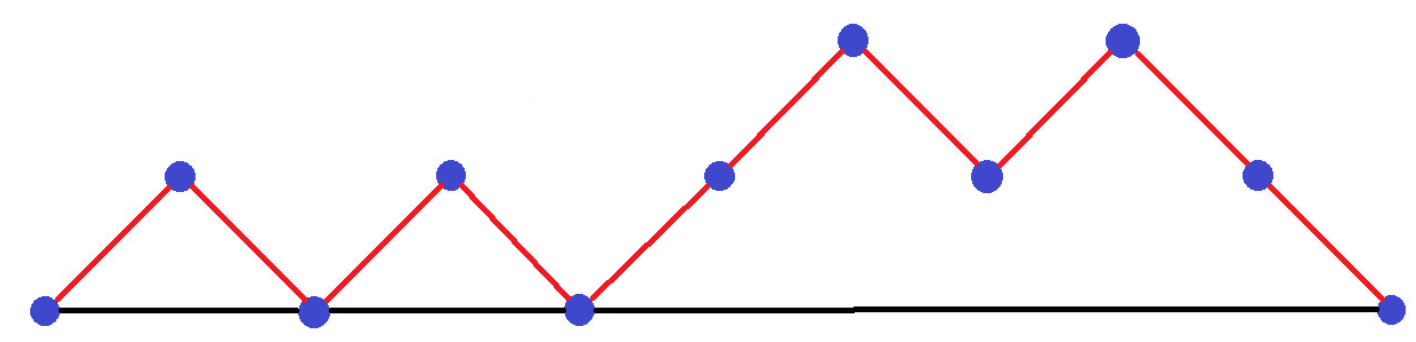

Figure 2.7: Illustration of a Dyck path for $k=5$.

\section{6}

\section{Statistical properties of the eigenvalues spectrum}

The probabilistic properties of the eigenvalue distribution allow to explore all the possible interpretations and applications of the RMT.

In the physics and mathematics, it is natural to study the expectation values of functions $f$ of the eigenvalues

$$
\mathrm{E}[f]=\langle f\rangle=\frac{1}{Z_{N}} \int \Pi_{i<j}\left(\lambda_{i}-\lambda_{j}\right)^{2} f\left(\lambda_{1}, \lambda_{2}, \ldots, \lambda_{N}\right) \Pi_{i=1}^{N} e^{-N V\left(\lambda_{i}\right)} d \lambda_{i} .
$$


One of the quantities of interest is the eigenvalue density that was defined in the preceding section for GUE. In general, it is defined in mathematics and physics notation respectively as

$$
\rho_{N}(\lambda)=\mathrm{E}\left[\frac{1}{N} \sum_{i=1}^{N} \delta\left(\lambda-\lambda_{i}\right)\right]=\left\langle\frac{1}{N} \sum_{i=1}^{N} \delta\left(\lambda-\lambda_{i}\right)\right\rangle,
$$

where both average are calculated with respect to jpdf on $\mathrm{R}$. Another point that one notice is the factor $\frac{1}{N}$ necessary to support a finite large $N$ limit of the eigenvalue density,

$$
\rho(\lambda)=\lim _{N \rightarrow \infty} \rho_{N}(\lambda)
$$

if this limit exists we obtain the equilibrium density. Existence of this limit generally depends on the behaviour of $V$ at infinity. Just like $N \rho(\lambda) d \lambda$ counts the average number of energy levels between $\lambda$ and $\lambda+d \lambda$, thus the mean spacing between consecutive eigenvalues is given by

$$
\Delta=\frac{1}{N \rho(\lambda)}
$$

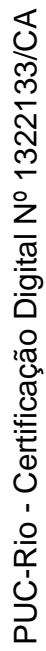

Another quantity of interest is the 2-point correlation function,

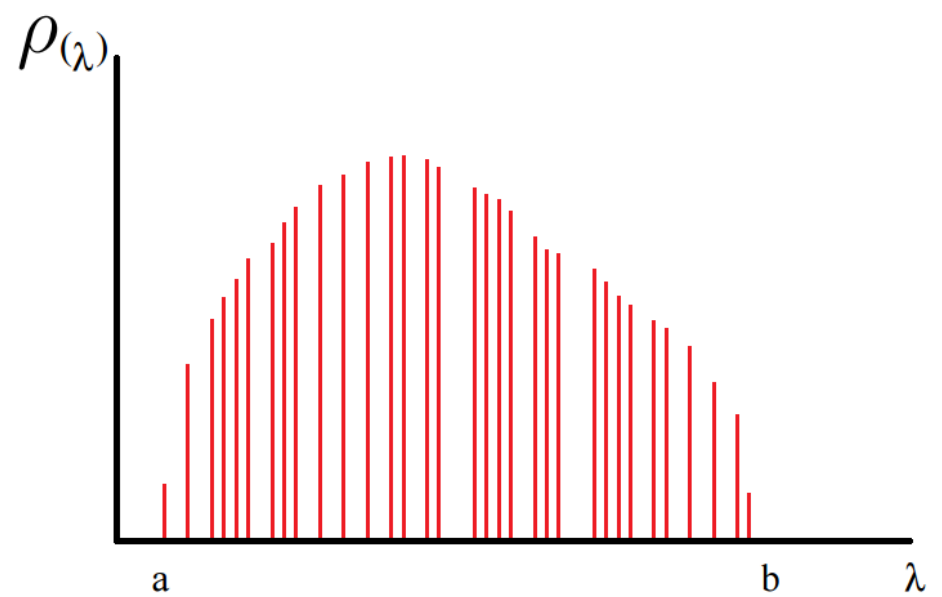

Figure 2.8: Typical eigenvalue distribution for $N$ finite.

$$
\begin{aligned}
\rho_{2, N}\left(\lambda_{1}, \lambda_{2}\right)= & \left\langle\frac{1}{N} \sum_{i, j=1}^{N} \delta\left(\lambda_{1}-\lambda_{i}\right) \delta\left(\lambda_{2}-\lambda_{j}\right)\right\rangle \\
& -\left\langle\frac{1}{N} \sum_{i=1}^{N} \delta\left(\lambda_{1}-\lambda_{i}\right)\right\rangle\left\langle\frac{1}{N} \sum_{i=1}^{N} \delta\left(\lambda_{2}-\lambda_{i}\right)\right\rangle,
\end{aligned}
$$

where $\rho_{2, N}\left(\lambda_{1}, \lambda_{2}\right) d \lambda_{1} d \lambda_{2}$ corresponds to the probability that there is one eigenvalue in $\left[\lambda_{1}, \lambda_{1}+d \lambda_{1}\right]$ and one in $\left[\lambda_{2}, \lambda_{2}+d \lambda_{2}\right]$. In the large $N$ limit this correlation function is denoted by

$$
\rho_{2}\left(\lambda_{1}, \lambda_{2}\right)=\lim _{N \rightarrow \infty} \rho_{2, N}\left(\lambda_{1}, \lambda_{2}\right)
$$


In fact, this function only depends on the complex structure of the resolvent function and can be obtained by Riemann Hilbert approach. Indeed we will further obtain the scaling limit of this correlation function in the bulk (far from the end points) of the spectrum which for any $\lambda \in(a, b)$ is given by [13] [17] [19]

$$
\rho_{2}\left(\lambda+\Delta \lambda_{1}, \lambda+\Delta \lambda_{2}\right) \sim \frac{\sin ^{2}\left[\pi\left(\lambda_{1}-\lambda_{2}\right)\right]}{\left[\pi\left(\lambda_{1}-\lambda_{2}\right)\right]^{2}} .
$$

In general, the $n$-point correlation function is given as

$$
\rho_{n}\left(\lambda_{1}, \ldots, \lambda_{n}\right)=\lim _{N \rightarrow \infty} N^{n-2}\left\langle\prod_{k}^{n} \sum_{i=1}^{N} \delta\left(\lambda_{k}-\lambda_{i}\right)\right\rangle_{c},
$$

where the index "c" restricts only to connected correlations. Also if this limit exists it depends on the complex structure of the resolvent function, such that can be approached by Riemann Hilbert problem and expressed in the bulk of the spectrum as

$$
\rho_{n}\left(\lambda+\lambda_{1}, \ldots, \lambda+\lambda_{n}\right) \sim\left|\begin{array}{ccc}
\frac{\sin \left[\pi\left(\lambda_{1}-\lambda_{1}\right)\right]}{\left[\pi\left(\lambda_{1}-\lambda_{1}\right)\right]} & \ldots & \frac{\sin \left[\pi\left(\lambda_{1}-\lambda_{n}\right)\right]}{\left[\pi\left(\lambda_{1}-\lambda_{n}\right)\right]} \\
\vdots & \ddots & \vdots \\
\frac{\sin \left[\pi\left(\lambda_{n}-\lambda_{1}\right)\right]}{\left[\pi\left(\lambda_{n}-\lambda_{1}\right)\right]} & \cdots & \frac{\sin \left[\pi\left(\lambda_{n}-\lambda_{n}\right)\right]}{\left[\pi\left(\lambda_{n}-\lambda_{n}\right)\right]}
\end{array}\right|^{2} .
$$

So far, we have considered scaling limits away from the endpoints. However, there exist a different scaling of the 2-point correlation function near the endpoints, and for $c>0$ are given by [17]

$$
\begin{aligned}
& \lim _{N \rightarrow \infty} \rho_{2, N}\left(a-\frac{\lambda_{1}}{(N c)^{\frac{2}{3}}}, a-\frac{\lambda_{2}}{(N c)^{\frac{2}{3}}}\right) \sim \frac{\left(A i\left(\lambda_{1}\right) A i^{\prime}\left(\lambda_{2}\right)-A i^{\prime}\left(\lambda_{1}\right) A i\left(\lambda_{2}\right)\right)^{2}}{\left(\lambda_{1}-\lambda_{2}\right)^{2}} \\
& \lim _{N \rightarrow \infty} \rho_{2, N}\left(b+\frac{\lambda_{1}}{(N c)^{\frac{2}{3}}}, b+\frac{\lambda_{2}}{(N c)^{\frac{2}{3}}}\right) \sim \frac{\left(A i\left(\lambda_{1}\right) A i^{\prime}\left(\lambda_{2}\right)-A i^{\prime}\left(\lambda_{1}\right) A i\left(\lambda_{2}\right)\right)^{2}}{\left(\lambda_{1}-\lambda_{2}\right)^{2}}
\end{aligned}
$$

where $\operatorname{Ai}(x)$ is the Airy function. This scaling limit will be obtained by the Riemann Hilbert approach. 


\section{3}

\section{The Dyson gas and eigenvalue density}

Dyson gas is an useful analogy that allows to understand the joint probability distribution function ( $j p d f$ ) of the eigenvalues. In this chapter we introduce a Dyson gas in thermodynamical equilibrium. A Dyson gas with Brownian motion is shown such that approaches to GUE for long enough time. We discuss the Dyson gas in equilibrium by the saddle point equation. Furthermore, using the resolvent function one can solve this equation. Alternatively we rephrase both saddle point equation and resolvent function as Riemann Hilbert problems. In the second section of this chapter we follow the exposition of [31], while the third section is based on the text [1]. For more detail the reader should consult [21].

\section{1}

Introduction

Our main goal here is to understand the spectrum of a random matrix ensemble as stationary and dynamical system. There is an analogy with a gas of eigenvalues in a potential well. The analogy is available only when one is able to integrate out the angular degrees of freedom and write the integral only in terms of eigenvalues. As a consequence of integrating out the eigenvector components, a term $\Pi_{i<j}\left(\lambda_{i}-\lambda_{j}\right)^{2}$ appears, which is responsible for the strong correlations (long-range) among the eigenvalues. The presence of this term leads to a natural and far-reaching interpretation of the set of $\mathrm{N}$ real eigenvalues as positions for $\mathrm{N}$ particles of a $1 \mathrm{D}$ fluid and subject to a logarithmic (Coulomb) repulsion plus a confining external potential. If we assume the Coulomb gas as a dynamical system, originally due to Dyson, one can actually infer the equilibrium positions of Coulomb particles in Brownian motion which correspond to the large $N$ limit of the jpdf of this particles, and hence to the jpdf of the eigenvalues. We obtain a resolvent function which can be represented via Riemann Hilbert problems. In fact, the saddle point equation can be rephrased as Riemann Hilbert problem too. These show the importance of Riemann Hilbert problems in the spectrum of random matrix theory.

Outline: The chapter is organized as follows. Section 3.2 contains the relation 
between the jpdf and Dyson gas via an equilibrium equation which is called saddle point equation. In section 3.3 we describe a nonequilibrium Dyson gas for GUE. Section 3.4 gives the solution of the saddle point equation which corresponds to the resolvent function. In section 3.5 we translate the saddle point equation and resolvent function as Riemann Hilbert problem. Showing the usefulness of the RHP.

\section{2}

\section{Dyson gas}

Raising the term $\Pi_{i<j}\left(\lambda_{i}-\lambda_{j}\right)^{2}$ into the exponent in the jpdf yields

$$
Z_{N}=\int \Pi_{i=1}^{N} d \lambda_{i} e^{-N^{2} S\left(\lambda_{1}, \ldots, \lambda_{N}\right)}
$$

where $S(\lambda)=\frac{1}{N} V(\lambda)-\frac{2}{N^{2}} \sum_{i<j} \ln \left|\lambda_{i}-\lambda_{j}\right|$ is the so-called effective action. This gives the Dyson gas: a charge distribution in 1D under the influence of a Coulomb repulsive force inside a confining potential $V(\lambda)$, in thermodynamical equilibrium at a temperature $k T=N^{-2}$. The equilibrium equation of this gas is given by

$$
\left.\frac{\delta S(\lambda)}{\delta \lambda_{i}}\right|_{\lambda_{i}=\tilde{\lambda}_{i}}=\frac{1}{N} V^{\prime}(\tilde{\lambda})-\frac{2}{N^{2}} \sum_{i \neq j} \frac{1}{\tilde{\lambda}_{i}-\tilde{\lambda}_{j}}=0 .
$$

From this one can for example obtain the eigenvalues density $\rho(\lambda)$ of the equilibrium measure when $N \rightarrow \infty$. In the case of the Gaussian Unitary

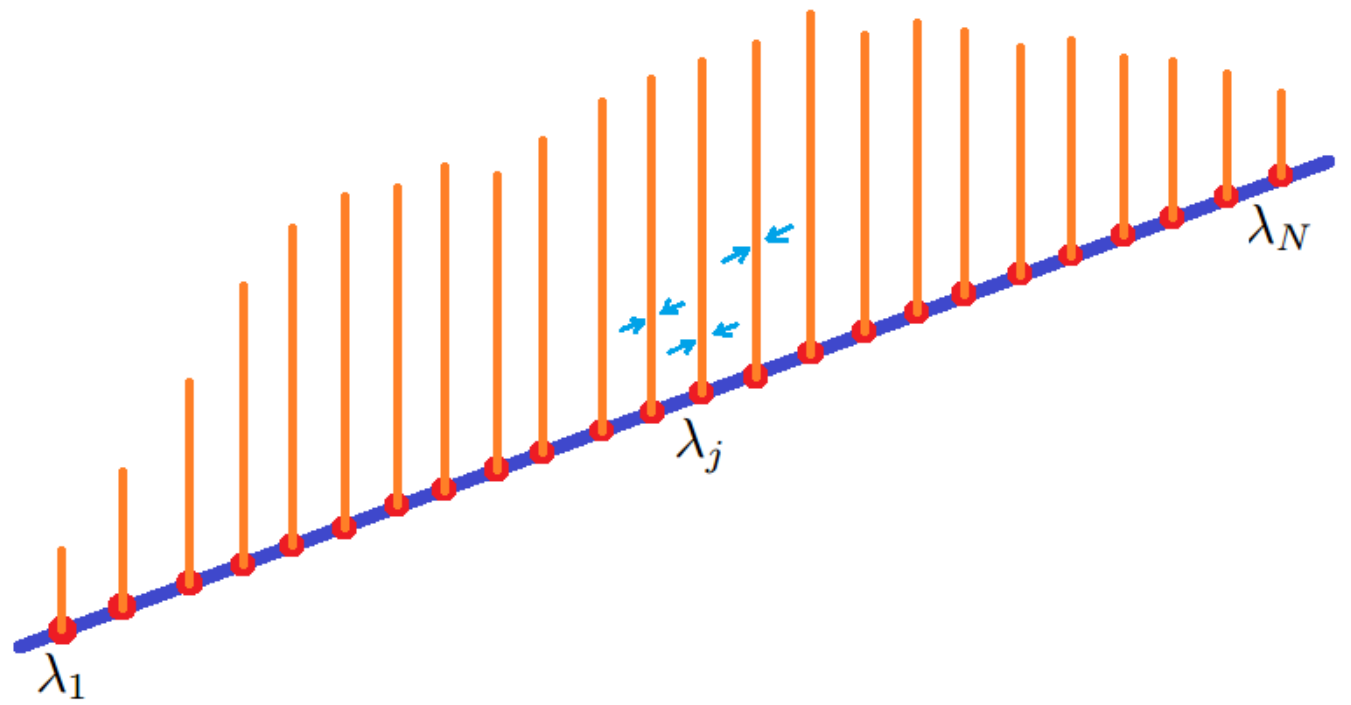

Figure 3.1: Pedagogycal illustration of the Dyson gas where one finds the repulsion among the particles and the probability distribution of all particles. 
Ensemble, with $V(\lambda)=\frac{\lambda^{2}}{2}$, this yields

$$
-\frac{2}{N} \sum_{i \neq j} \frac{1}{\tilde{\lambda}_{i}-\tilde{\lambda}_{j}}=\tilde{\lambda}
$$

which gives the Wigner's semi-circle law $\rho(\lambda)=\frac{1}{2 \pi} \sqrt{4-\lambda^{2}}$ as will be shown below. So that this describes the equilibrium of the charge distribution.

\section{3}

\section{Dyson-Nonstationary ensemble}

In the description of the Dyson gas and eigenvalue density, the distribution was assumed to be stationary. Now the Dyson gas is interpreted as a dynamical system that corresponds to nonequilibrium state depending on time until the equilibrium is approached. Dyson propoused that for GUE, $\lambda_{i}$ should be interpreted as positions of particles in Brownian motion [1] [25]. This Brownian particle is subject to random collisions with other particles, so that these collisions give rise to an average frictional force resisting their motion. Hence this force will be characterized by a friction coefficient $\eta$. While the particle at $\lambda_{i}$ experiences an external force field,

$$
F\left(\lambda_{i}\right)=-\frac{\delta S(\lambda)}{\delta \lambda_{i}}=-\frac{1}{N} \lambda_{i}+\frac{2}{N^{2}} \sum_{i \neq j} \frac{1}{\lambda_{i}-\lambda_{j}} .
$$

Under these physical conditions, the Langevin equation is given by:

$$
\ddot{\lambda}_{i}=-\eta \dot{\lambda}_{i}+F\left(\lambda_{i}\right)+f(t)
$$

where the stochastic force $f(t)$ obeys the following properties

$$
\begin{aligned}
\left\langle f\left(t_{1}\right) f\left(t_{2}\right) \ldots f\left(t_{2 n+1}\right)\right\rangle & =0 \\
\left\langle f\left(t_{1}\right) f\left(t_{2}\right) \ldots f\left(t_{2 n}\right)\right\rangle & =\sum_{\text {pairs }}\left\langle f\left(t_{i}\right) f\left(t_{j}\right)\right\rangle \ldots\left\langle f\left(t_{k}\right) f\left(t_{l}\right)\right\rangle \\
\left\langle f\left(t_{1}\right) f\left(t_{2}\right)\right\rangle & =2 \eta k T \delta\left(t_{1}-t_{2}\right) .
\end{aligned}
$$

To characterize the higher moments of $f(t)$, we assume that $f(t)$ follows a Gaussian distribution

$$
P[f(t)]=e^{-\int_{t_{0}}^{t_{f}} d t \frac{f(t)^{2}}{4 \eta k T}}
$$

where this ditribution gives the probability density for the values of $f(t)$ in the interval $\left[t_{0}, t_{f}\right]$.

For the Langevin equation, considering that the evolution of the system is a Markov process, we can also define a probability density

$$
P\left(\lambda_{1}, \lambda_{2}, \ldots, \lambda_{N} ; t\right)=\left\langle\delta\left(\lambda_{1}-x_{1}(t)\right) \delta\left(\lambda_{2}-x_{2}(t)\right) \ldots \delta\left(\lambda_{N}-x_{N}(t)\right)\right\rangle,
$$


where these $x_{i}(t)$ obey the Langevin equation and $P\left(\lambda_{1}, \lambda_{2}, \ldots, \lambda_{N} ; t\right) d \lambda_{1} d \lambda_{2} \ldots d \lambda_{N}$ is the probability of finding the particle at time $t$ within the intervals $\left[\lambda_{1}, \lambda_{1}+d \lambda_{1}\right] \cup\left[\lambda_{2}, \lambda_{2}+d \lambda_{2}\right] \cup \ldots \cup\left[\lambda_{N}, \lambda_{N}+d \lambda_{N}\right]$. We now derive the Smoluchowski equation for $P\left(\lambda_{1}, \lambda_{2}, \ldots, \lambda_{N} ; t\right)$, through the next steps:

$\frac{\partial}{\partial t} P\left(\lambda_{1}, \lambda_{2}, \ldots, \lambda_{N} ; t\right)=-\sum_{i}^{N} \frac{\partial}{\partial \lambda_{i}}\left\langle\delta\left(\lambda_{1}-x_{1}(t)\right) \delta\left(\lambda_{2}-x_{2}(t)\right) \ldots \delta\left(\lambda_{N}-x_{N}(t)\right) \dot{x}_{i}(t)\right\rangle$

by (3.4), we have that

$$
\begin{aligned}
\frac{\partial}{\partial t} P(\lambda, t)= & -\sum_{i}^{N} \frac{\partial}{\partial \lambda_{i}}\left\langle\delta\left(\lambda_{1}-x_{1}(t)\right) \delta\left(\lambda_{2}-x_{2}(t)\right) \ldots \delta\left(\lambda_{N}-x_{N}(t)\right) \frac{1}{\eta}\left(F\left(x_{i}\right)+f(t)\right)\right\rangle \\
= & -\frac{1}{\eta} \sum_{i}^{N} \frac{\partial}{\partial \lambda_{i}}\left(P\left(\lambda_{1}, \lambda_{2}, \ldots, \lambda_{N} ; t\right) F\left(\lambda_{i}\right)\right)- \\
& -\frac{1}{\eta} \sum_{i}^{N} \frac{\partial}{\partial \lambda_{i}}\left\langle\delta\left(\lambda_{1}-x_{1}(t)\right) \delta\left(\lambda_{2}-x_{2}(t)\right) \ldots \delta\left(\lambda_{N}-x_{N}(t)\right) f(t)\right\rangle \\
= & -\frac{1}{\eta} \sum_{i}^{N} \frac{\partial}{\partial \lambda_{i}}\left(P\left(\lambda_{1}, \lambda_{2}, \ldots, \lambda_{N} ; t\right) F\left(\lambda_{i}\right)\right)- \\
& -\frac{2 \eta k T}{\eta} \sum_{i}^{N} \frac{\partial}{\partial \lambda_{i}}\left\langle\frac{\delta}{\delta f(t)} \delta\left(\lambda_{1}-x_{1}(t)\right) \delta\left(\lambda_{2}-x_{2}(t)\right) \ldots \delta\left(\lambda_{N}-x_{N}(t)\right)\right\rangle \\
= & -\frac{1}{\eta} \sum_{i}^{N} \frac{\partial}{\partial \lambda_{i}}\left(P\left(\lambda_{1}, \lambda_{2}, \ldots, \lambda_{N} ; t\right) F\left(\lambda_{i}\right)\right)+ \\
& +2 k T \sum_{i}^{N} \frac{\partial^{2}}{\partial \lambda_{i}^{2}}\left\langle\delta\left(\lambda_{1}-x_{1}(t)\right) \delta\left(\lambda_{2}-x_{2}(t)\right) \ldots \delta\left(\lambda_{N}-x_{N}(t)\right) \frac{\delta x_{i}(t)}{\delta f(t)}\right\rangle \\
= & -\frac{1}{\eta} \sum_{i}^{N} \frac{\partial}{\partial \lambda_{i}}\left(P\left(\lambda_{1}, \lambda_{2}, \ldots, \lambda_{N} ; t\right) F\left(\lambda_{i}\right)\right)+ \\
& +k T \sum_{i}^{N} \frac{\partial^{2}}{\partial \lambda_{i}^{2}} P\left(\lambda_{1}, \lambda_{2}, \ldots, \lambda_{N} ; t\right) \\
& \\
& \\
&
\end{aligned}
$$

hence, we obtain

$$
\begin{aligned}
\eta \frac{\partial}{\partial t} P\left(\lambda_{1}, \lambda_{2}, \ldots, \lambda_{N} ; t\right)= & \sum_{i}^{N}\left(-\frac{\partial}{\partial \lambda_{i}}\left(P\left(\lambda_{1}, \lambda_{2}, \ldots, \lambda_{N} ; t\right) F\left(\lambda_{i}\right)\right)+\right. \\
& \left.+\eta k T \frac{\partial^{2}}{\partial \lambda_{i}^{2}} P\left(\lambda_{1}, \lambda_{2}, \ldots, \lambda_{N} ; t\right)\right) .
\end{aligned}
$$

Notice that one can express the Smoluchowski equation in the form of an equation of continuity 


$$
\frac{\partial}{\partial t} P\left(\lambda_{1}, \lambda_{2}, \ldots, \lambda_{N} ; t\right)=-\sum_{i}^{N} \frac{\partial}{\partial \lambda_{i}} j\left(\lambda_{1}, \lambda_{2}, \ldots, \lambda_{N} ; t\right)
$$

with the current density

$$
j\left(\lambda_{1}, \lambda_{2}, \ldots, \lambda_{N} ; t\right)=-\frac{1}{\eta}\left(\eta k T \frac{\partial}{\partial \lambda_{i}}-F\left(\lambda_{i}\right)\right) P\left(\lambda_{1}, \lambda_{2}, \ldots, \lambda_{N} ; t\right),
$$

where the current density $j\left(\lambda_{1}, \lambda_{2}, \ldots, \lambda_{N} ; t\right)$ contains a diffusion term and a drift term. In this case the continuity equation reflects the fact that the particle is always somewhere at the positions $\lambda_{1}, \lambda_{2}, \ldots, \lambda_{N}$ and that it moves by a continuous motion.

Since there exists a unique solution to the Smoluchowski equation which is independent of time, this time independent solution is given by the probability distribution of the GUE.

Analogously to the eigenvalue distribution we can interpret that the matrix elements $M_{i j}$ obey a Brownian process. This process is defined by requiring that the $N^{2}$ random variables $M_{k}=\left\{M_{i i}, \Re M_{i j}, \Im M_{i j}\right\}$ have moments

$$
\begin{gathered}
\left\langle M_{k}\right\rangle=-\eta M_{k}, \\
\left\langle\left(M_{k}\right)^{2}\right\rangle=g_{i j} \eta k T,
\end{gathered}
$$

where $g_{i j}=1+\delta_{i j}$. In this Brownian motion the matrix elements $M_{k}$ are completely uncoupled, as was studied in the section GUE, and each is subject only to harmonic force. As before, the Smoluchowski equation corresponding to this Brownian force is given by

$$
\eta \frac{\partial P}{\partial t}=\sum_{k=1}^{N^{2}}\left[\frac{1}{2} g_{i j} \eta k T \frac{\partial^{2} P}{\partial M_{k}^{2}}+\frac{\partial}{\partial M_{k}}\left(M_{k} P\right)\right] .
$$

Here $P\left(M_{1}, M_{2}, \ldots, M_{N^{2}} ; t\right)$ is the time dependent probability density of the matrix elements $M_{k}$, so that the following solution satisfies the Smoluchowski equation with initial condition $M=M^{\prime}$ at $t=0$,

$$
P\left(M_{1}, M_{2}, \ldots, M_{N^{2}} ; t\right)=C\left(1-p^{2}\right)^{-\frac{N^{2}}{2}} e^{-\frac{T r\left(M-p M^{\prime}\right)^{2}}{2 \eta k T\left(1-p^{2}\right)}},
$$

where $\mathrm{C}$ is a normalization constant and $p=e^{-\frac{t}{\eta}}$. Consequently, just after $t \rightarrow \infty$ we have $p \rightarrow 0$, the stationary probability distribution is given by

$$
P\left(M_{1}, M_{2}, \ldots, M_{N^{2}} ; t\right)=C e^{-\frac{T r(M)^{2}}{2 \eta k T}}
$$

which is the Gibbs measure for the GUE. And this measure is the unique equilibrium measure for the Brownian process. 
Therefore when all the matrix elements execute Brownian motion according to

$$
\begin{gathered}
\left\langle M_{k}\right\rangle=-\eta M_{k}, \\
\left\langle\left(M_{k}\right)^{2}\right\rangle=g_{i j} \eta k T,
\end{gathered}
$$

with arbitrary initial values, its eigenvalues $\left\{\lambda_{i}\right\}$ execute also a Brownian motion with the Smoluchowski equation (3.7), which gives the jpdf of the GUE after a long enough time.

\section{4}

\section{Saddle point equation}

In this section we will rephrase the saddle point equation as second order equation for a so-called resolvent function. Recall that the saddle point equation is given by

$$
\frac{1}{N} V^{\prime}(\tilde{\lambda})-\frac{2}{N^{2}} \sum_{i \neq j} \frac{1}{\tilde{\lambda}_{i}-\tilde{\lambda}_{j}}=0,
$$

where the eigenvalues do not accumulate in the minimun of the potential due to the term $\sum_{i \neq j} \frac{1}{\hat{\lambda}_{i}-\tilde{\lambda}_{j}}$. Multiplying each term of the saddle point equation by $\frac{1}{z-\tilde{\lambda_{i}}}$ and summing over $i$, we have

$$
\begin{aligned}
\frac{1}{N} \sum_{i=1}^{N} V^{\prime}(\tilde{\lambda}) \frac{1}{z-\tilde{\lambda}_{i}}-\frac{2}{N^{2}} \sum_{i=1}^{N} \sum_{i \neq j} \frac{1}{\tilde{\lambda}_{i}-\tilde{\lambda}_{j}} \frac{1}{z-\tilde{\lambda}_{i}} & =0 \\
\frac{1}{N} \sum_{i=1}^{N} V^{\prime}(\tilde{\lambda}) \frac{1}{z-\tilde{\lambda}_{i}}-\frac{1}{N^{2}} \sum_{i=1}^{N} \sum_{i \neq j} \frac{1}{\tilde{\lambda}_{i}-\tilde{\lambda}_{j}}\left(\frac{1}{z-\tilde{\lambda}_{i}}-\frac{1}{z-\tilde{\lambda}_{j}}\right) & =0 \\
\frac{1}{N} \sum_{i=1}^{N} V^{\prime}(\tilde{\lambda}) \frac{1}{z-\tilde{\lambda}_{i}}-\frac{1}{N^{2}} \sum_{i=1}^{N} \sum_{i \neq j} \frac{1}{\left(z-\tilde{\lambda}_{i}\right)\left(z-\tilde{\lambda}_{j}\right)} & =0 \\
\frac{1}{N} \sum_{i=1}^{N} V^{\prime}(\tilde{\lambda}) \frac{1}{z-\tilde{\lambda}_{i}}-\frac{1}{N^{2}} \sum_{i, j=1}^{N} \frac{1}{\left(z-\tilde{\lambda}_{i}\right)\left(z-\tilde{\lambda}_{j}\right)}-\frac{1}{N^{2}} \sum_{i=1}^{N} \frac{1}{\left(z-\tilde{\lambda}_{i}\right)^{2}} & =0 .
\end{aligned}
$$

Upon adding and subtracting the term $\frac{1}{N} \sum_{i=1}^{N} V^{\prime}(z) \frac{1}{z-\hat{\lambda}_{i}}$, we have

$$
\begin{aligned}
& \frac{1}{N} \sum_{i=1}^{N} V^{\prime}(z) \frac{1}{z-\tilde{\lambda}_{i}}+\frac{1}{N} \sum_{i=1}^{N} \frac{V^{\prime}(\tilde{\lambda})-V^{\prime}(z)}{z-\tilde{\lambda}_{i}}- \\
& -\frac{1}{N^{2}} \sum_{i, j=1}^{N} \frac{1}{\left(z-\tilde{\lambda}_{i}\right)\left(z-\tilde{\lambda}_{j}\right)}-\frac{1}{N^{2}} \sum_{i=1}^{N} \frac{1}{\left(z-\tilde{\lambda}_{i}\right)^{2}}=0,
\end{aligned}
$$

if we define, $\omega(z)=\frac{1}{N} \sum_{i=1}^{N} \frac{1}{z-\tilde{\lambda}_{i}}$, this equation can be rewritten as

$$
V^{\prime}(z) \omega(z)-P(z)-\omega(z)^{2}+\frac{1}{N} \omega^{\prime}(z)=0,
$$


where, $P(z)=\frac{1}{N} \sum_{i=1}^{N} \frac{V^{\prime}(z)-V^{\prime}(\tilde{\lambda})}{z-\tilde{\lambda}_{i}}$, which is a polynomial of degree $d-2$ when $V(z)$ is of degree $d$.

In the large $N$ limit, we only consider $\mathrm{O}(1)$ terms

$$
\omega(z)^{2}-V^{\prime}(z) \omega(z)+P(z)=0
$$

whose solution is given by

$$
\omega(z)=\frac{V^{\prime}(z) \pm \sqrt{V^{\prime 2}(z)-4 P(z)}}{2}
$$

One notes that $\omega(z)$ behaves like $\sim \frac{1}{z}$ for $|z|$ large by its definition. Hence the sign "-" in the solution allows for the cancelling of the higher orders than $O\left(\frac{1}{z}\right)$ for large $|z|$. Thus one gets

$$
\omega(z)=\frac{V^{\prime}(z)-\sqrt{V^{\prime 2}(z)-4 P(z)}}{2} .
$$

In the context of this thesis, we consider the one cut solution. In this case, $V(z)$ has only one minimum, thence we have a single connected cut $[a, b]$ and only two branch points $a$ and $b$ in the complex plane. This one cut condition is enough to determine $P(z)$ and the branch points $a$ and $b$, because in

$$
V^{\prime 2}(z)-4 P(z)=M^{2}(z)(z-a)(z-b) .
$$

There are as many unknowns coefficients of $P$, of $M, a$ and $b$ as well as equations for each order of $z$. Therefore, $\omega(z)$ becomes

$$
\omega(z)=\frac{V^{\prime}(z)-M(z) \sqrt{(z-a)(z-b)}}{2}
$$

which is known as the resolvent function. We will note that this function gives statistical properties of the equilibrium eigenvalue distribution in the next section.

For the case of the GUE, $V(z)=\frac{z^{2}}{2}$. we have that $V^{\prime}(z)=z$ then $P(z)$ is of degree 0 , in particular $P(z)=c$. Inserting these terms in the equation (3.16) and considering $M(z)=d$ to have the same order in both sides of this equation, we obtain

$$
V^{\prime 2}(z)-4 P(z)=z^{2}-4 c=M^{2}(z)(z-a)(z-b)=d^{2}(z-a)(z-b)
$$

Since the potential is even, then $b=-a$. Therefore

$$
z^{2}-4 c=d^{2}\left(z^{2}-a^{2}\right)
$$


Here we observe that $d^{2}=1=M^{2}$ and in particular if $c=1$ then $a=2$. Hence the resolvent function is given by

$$
\omega(z)=\frac{z-\sqrt{(z-2)(z+2)}}{2}
$$

such that the eigenvalue density corresponds to

$$
\rho(z)=\frac{1}{2 \pi} \sqrt{(2-z)(z+2)}
$$

\section{5}

\section{Resolvent function as a Riemann Hilbert representation}

In the Random Matrix Theory, it is common to consider the resolvent function as the generating function of moments

$$
\omega(z)=\frac{1}{N} \sum_{k=0}^{\infty} \frac{\left\langle\operatorname{Tr} M^{k}\right\rangle}{z^{k+1}},
$$

thus the generating function $\omega(z)$ encodes all the statistical information of the spectrum of $M$. If we define $\omega_{k}=\frac{1}{N}\left\langle\operatorname{Tr} M^{k}\right\rangle$, then one has a Laurent series

$$
\omega(z)=\sum_{k=0}^{\infty} \frac{\omega_{k}}{z^{k+1}} .
$$

One additional feature is that $\omega_{k}$ generates surfaces with one boundary of length $k$, so that $\omega(z)$ is the generating function for surfaces with one boundary of arbitrary length.

From other point of view $\omega(z)$ is a Laurent series, hence by inverse discrete Laplace transformation, $\omega_{k}$ is given by

$$
\omega_{k}=-\frac{1}{2 \pi i} \oint \omega(z) z^{k} d z
$$

where the contour is a clockwise circle around infinity. In the definition, $\omega(z)$ becomes singular when $z$ approaches each $\lambda_{i}$, but we can analytically extend this function to the whole complex plane in particular when $N \rightarrow \infty$. In this limit there are many eigenvalues such that we can consider the one cut solution. Therefore $\omega(z)$ is discontinuous across $[a, b]$.

Now let consider a function $\rho$ defined on $[a, b]$ which determine the jump condition for $\omega(z)$, given by

$$
\omega(\lambda+i \epsilon)=\omega(\lambda-i \epsilon)-2 \pi i \rho(z) .
$$

So that we can define the resolvent function $\omega(z)$ by a Riemann Hilbert representation that satisfies the following: 
1. $\omega(z)$ is analytic on $\mathrm{C} \backslash[a, b]$.

2. For any $\lambda \in[a, b]$,

$$
\lim _{\epsilon \rightarrow 0^{+}} \omega(\lambda+i \epsilon)=\lim _{\epsilon \rightarrow 0^{+}} \omega(\lambda-i \epsilon)-2 \pi i \rho(\lambda)
$$

3. As $z \rightarrow \infty$,

$$
\omega(z) \cong \frac{1}{z}
$$

There is a unique function which satisfies these requirements which is

$$
\omega(z)=\frac{1}{2 \pi i} \int_{[a, b]} \frac{-2 \pi i \rho(\lambda)}{\lambda-z} d \lambda=\int_{[a, b]} \frac{\rho(\lambda)}{z-\lambda} d \lambda .
$$

Note that this is analogue to the definition of $\omega(z)$ when the distribution of eigenvalues becomes continuous.

In addition at the large $N$ limit, the saddle point equation can be rewritten as a functional of the eigenvalue density $\rho(\lambda)$,

$$
V^{\prime}(\lambda)-2 f \frac{\rho\left(\lambda^{\prime}\right) d \lambda^{\prime}}{\lambda-\lambda^{\prime}}=0
$$

by the definition of the principal value integral,

$$
\begin{aligned}
V^{\prime}(\lambda)-\lim _{\epsilon \rightarrow 0^{+}}\left(\int_{[a, b]-C_{\epsilon}} \frac{\rho\left(\lambda^{\prime}\right)}{\lambda-i \epsilon-\lambda^{\prime}} d \lambda^{\prime}+\int_{[a, b]+C_{\epsilon}} \frac{\rho\left(\lambda^{\prime}\right)}{\lambda+i \epsilon-\lambda^{\prime}} d \lambda^{\prime}\right)=0 \\
V^{\prime}(\lambda)-\lim _{\epsilon \rightarrow 0^{+}}\left(\int_{[a, b]} \frac{\rho\left(\lambda^{\prime}\right)}{\lambda-i \epsilon-\lambda^{\prime}} d \lambda^{\prime}+\int_{[a, b]} \frac{\rho\left(\lambda^{\prime}\right)}{\lambda+i \epsilon-\lambda^{\prime}} d \lambda^{\prime}\right)=0,
\end{aligned}
$$

thus by the definition (3.22) we obtain

$$
V^{\prime}(\lambda)-\lim _{\epsilon \rightarrow 0^{+}}(\omega(\lambda-i \epsilon)+\omega(\lambda+i \epsilon))=0 .
$$

Note that the simplest one cut solution for the respective homogeneous equation corresponds to $V^{\prime}(\lambda)=0$, and by (3.17) this solution is $\frac{\sqrt{(z-a)(z-b)}}{2}$, which is the singular part of $\omega(z)$. Hence, for transform (3.23) into a jump condition, it is convenient to divide $(3.24)$ by $i \sqrt{(z-a)(z-b)}$,

$$
\lim _{\epsilon \rightarrow 0^{+}} \frac{\omega(\lambda+i \epsilon)}{i \sqrt{(z-a)(z-b)}}=-\lim _{\epsilon \rightarrow 0^{+}} \frac{\omega(\lambda-i \epsilon)}{i \sqrt{(z-a)(z-b)}}+\frac{V^{\prime}(\lambda)}{i \sqrt{(z-a)(z-b)}} .
$$

Therefore the saddle point equation can also be expressed as a Riemann Hilbert problem with the following conditions

1. $\frac{\omega(z)}{i \sqrt{(z-a)(z-b)}}$ is analytic on the complex plane, except $[a, b]$ 
2. $\forall \lambda \in[a, b]$,

$$
\lim _{\epsilon \rightarrow 0^{+}} \frac{\omega(\lambda+i \epsilon)}{i \sqrt{(z-a)(z-b)}}=-\lim _{\epsilon \rightarrow 0^{+}} \frac{\omega(\lambda-i \epsilon)}{i \sqrt{(z-a)(z-b)}}+\frac{V^{\prime}(\lambda)}{i \sqrt{(z-a)(z-b)}}
$$

3. As $|z| \rightarrow \infty$

$$
\frac{\omega(z)}{i \sqrt{(z-a)(z-b)}} \cong \frac{1}{i z^{2}}+O\left(\frac{1}{z^{3}}\right)
$$

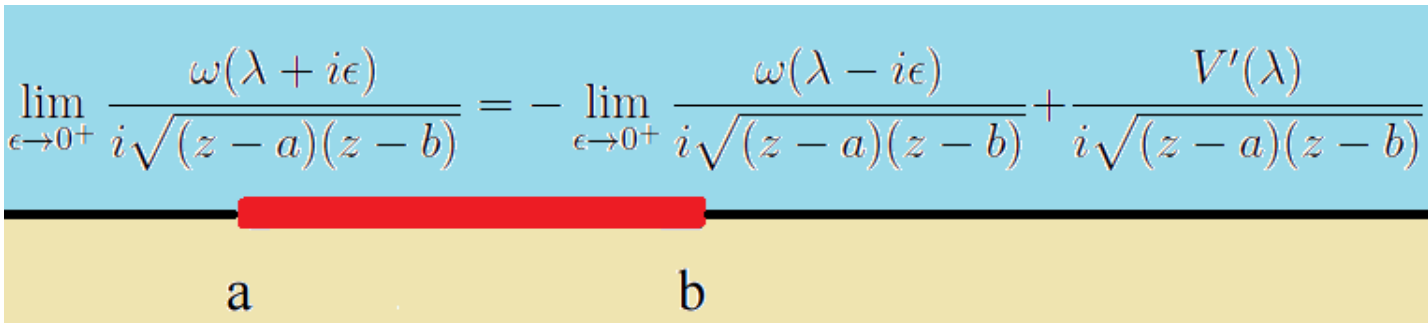

Figure 3.2: An illustration of the jump condition for the saddle point equation.

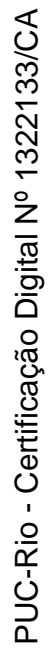

It gives the unique solution of the saddle point equation

$$
\frac{\omega(z)}{i \sqrt{(z-a)(z-b)}}=\frac{1}{2 \pi i} \int_{[a, b]} \frac{\frac{V^{\prime}(\lambda)}{i \sqrt{(b-\lambda)(\lambda-a)}} d \lambda}{\lambda-z},
$$

hence, the resolvent function is given by

$$
\omega(z)=\frac{\sqrt{(z-a)(z-b)}}{2 \pi} \int_{[a, b]} \frac{d \lambda}{\lambda-z} \frac{V^{\prime}(\lambda)}{\sqrt{(b-\lambda)(\lambda-a)}},
$$

so that if we expand this equation for $|z| \rightarrow \infty$ we find

$$
\begin{aligned}
& \omega(z)=\frac{z\left(1-\frac{(a+b)}{2 z}+O\left(\frac{1}{z^{2}}\right)\right)}{2 \pi} \int_{[a, b]} d \lambda \frac{1+\frac{\lambda}{z}}{z} \frac{V^{\prime}(\lambda)}{\sqrt{(b-\lambda)(\lambda-a)}} \\
& \omega(z)=\frac{1}{2 \pi} \int_{[a, b]} d \lambda \frac{V^{\prime}(\lambda)}{\sqrt{(b-\lambda)(\lambda-a)}}+\frac{1}{2 \pi} \int_{[a, b]} d \lambda \frac{\lambda}{z} \frac{V^{\prime}(\lambda)}{\sqrt{(b-\lambda)(\lambda-a)}}
\end{aligned}
$$

and then by the large $|z|$ behavior of the resolvent we have two other conditions for the coeficients of $\omega(z)$, such that

$$
\begin{aligned}
& \frac{1}{2 \pi} \int_{[a, b]} d \lambda \frac{V^{\prime}(\lambda)}{\sqrt{(b-\lambda)(\lambda-a)}}=0 \\
& \frac{1}{2 \pi} \int_{[a, b]} d \lambda \frac{\lambda V^{\prime}(\lambda)}{\sqrt{(b-\lambda)(\lambda-a)}}=1 .
\end{aligned}
$$

To emphasize the usefulness of the Riemann Hilbert approach we will demostrate that $P(z)$ is a polynomial, then through the jump condition of $\omega(z)$, 
we have

$$
\begin{aligned}
& P(\lambda+i \epsilon)=V^{\prime}(\lambda+i \epsilon) \omega(\lambda+i \epsilon)-\omega(\lambda+i \epsilon)^{2} \\
& P(\lambda-i \epsilon)=V^{\prime}(\lambda-i \epsilon) \omega(\lambda-i \epsilon)-\omega(\lambda-i \epsilon)^{2}
\end{aligned}
$$

subtracting both equations we obtain

$$
\begin{aligned}
P(\lambda+i \epsilon)-P(\lambda-i \epsilon)= & V^{\prime}(\lambda)(\omega(\lambda+i \epsilon)-\omega(\lambda-i \epsilon))+\omega(\lambda-i \epsilon)^{2}- \\
& -\omega(\lambda+i \epsilon)^{2} \\
= & \left(V^{\prime}(\lambda)-\omega(\lambda+i \epsilon)-\omega(\lambda-i \epsilon)\right)(\omega(\lambda+i \epsilon)- \\
& -\omega(\lambda-i \epsilon)) \\
P(\lambda+i \epsilon)-P(\lambda-i \epsilon)= & 0 .
\end{aligned}
$$

Thus $P(z)$ is analytic on C. Now since by definition $P(z) \cong \frac{V^{\prime}(z)}{z}$ for $z \rightarrow \infty$, we have that $P(z)$ is an entire function with polynomial behaviour at infinity, therefore $P(z)$ is a polynomial of degree $d-2$. 


\section{4}

\section{Orthogonal Polynomials}

Orthogonal polynomials have wide range of applications. In this chapter we review the generalities of orthogonal polynomials. From a combinatorial point of view, orthogonal polynomials can be represented by a three term recurrent relation. Analytically, the orthogonal polynomials are represented by a string equation which is showed below. We introduce the relation between the string equation and a staircase path. In this way, the orthogonal polynomials can be represented geometrically as staircase paths. Algebraically we represent orthogonal polynomials by commutation relation. Statistically the orthogonal polynomials are represented by a kernel and hence we compute the $n$-point correlation function of the eigenvalues as function of orthogonal polynomials. Furthermore, orthogonal polynomial can be represented as Riemann Hilbert problems that will be studied in the next chapter. In the third section of this chapter we follow the exposure of [3], while the fourth section is based on the text [29]. For more detail the reader should consult [21].

\section{1 \\ Introduction}

Orthogonal polynomials are a very interesting topic of analysis that have numerous links with other branches of analysis, spectral theory, number theory, mathematical physics, etc. One of the links is with Random Matrix Theory. Moreover, we can characterize orthogonal polynomials by Riemann Hilbert problem in this context.

By itself, orthogonal polynomials are an efficient tool to study the distribution of eigenvalues of unitary ensembles for any $N$. In particular, this tool is crucial for compute all the correlation functions in the large $N$ limit.

Outline: The chapter is organized as follows. In section 4.2 we described orthogonal polynomials. In section 4.3 we compute the partition function by orthogonal polynomials and derive the string equation. In section 4.4 we introduce staircase paths that represent terms of the string equation. Section 4.5 gives two operators that represent the string equation. This is followed by computing the $n$ point correlation function in the section 4.6. 


\section{2}

\section{Generalities of orthogonal polynomials}

Let $\wp$ be a real linear space of polynomials in one variable with real coefficients, with a positive definite scalar product $(\cdot, \cdot)$. By applying the Gram-Schimdt orthogonalization to monomials $\left\{1, \lambda, \lambda^{2}, \ldots\right\}$ one obtains mutually orthogo$n a l$ (with respect to the scalar product) polynomials $\left\{p_{0}(\lambda), p_{1}(\lambda), p_{2}(\lambda), \ldots\right\}$, such that

$$
\begin{aligned}
& p_{0}(\lambda)=1 \\
& p_{n}(\lambda)=\lambda^{n}-\sum_{i=0}^{n-1} \frac{\left(p_{i}, \lambda^{i}\right)}{\left(p_{i}, p_{i}\right)} p_{i}(\lambda) .
\end{aligned}
$$

Moreover, the subspace $\wp_{n}$ generated by $\left\{\lambda^{n}\right\}$ is the same as the subspace generated by $\left\{p_{n}(\lambda)\right\}$.

The uniqueness of the orthogonal polynomials is determined by the constants $h_{n}$ and $a_{n}$, in the following way

$$
\begin{aligned}
\left(p_{n}, p_{n}\right) & =h_{n} \\
p_{n}(\lambda) & =a_{n} \lambda^{n}+O\left(\lambda^{n-1}\right) .
\end{aligned}
$$

Furthermore, when one fixes to 1 some of this constants we have two types of orthogonal polynomials: orthonormal polynomials when $h_{n}=1$ and monic orthogonal polynomials when $a_{n}=1$. For our interest, we consider implicitly monic orthogonal polynomials, so that only $h_{n}$ establishes the uniqueness.

In particular, for the orthogonal polynomials $p_{n}, p_{m} \in \wp$, we have the scalar product of the form

$$
\left(p_{n}, p_{m}\right)=\int_{\mathrm{R}} p_{n}(\lambda) p_{m}(\lambda) d \mu(\lambda)
$$

The map $\mu: B \rightarrow[0, \infty)$ is a finite (Borel) measure on $\mathrm{R}$, such that $B$ corresponds to a system of Borel subsets of $\mathrm{R}$. That means that for any system of mutually disjoint sets $\left\{A_{i}\right\}$ one has

$$
\mu\left(\bigcup_{i=1}^{\infty} A_{i}\right)=\sum_{i=1}^{\infty} \mu\left(A_{i}\right) .
$$

The measure $\mu$ is absolutely continuous if there exists a positive function $w$ such that $d \mu(\lambda)=w(\lambda) d \lambda$. Then the scalar product acquires the following form

$$
\int_{\mathrm{R}} p_{n}(\lambda) p_{m}(\lambda) d \mu(\lambda)=\int_{\mathrm{R}} p_{n}(\lambda) p_{m}(\lambda) w(\lambda) d \lambda .
$$


Analyzing the above ideas, if $\mu$ is a absolutely continuous measure on $\mathrm{R}$ with distribution function $\mu(\lambda)=\mu((-\infty, \lambda])$, such that all the moments

$$
\int_{\operatorname{supp}(\mu)} \lambda^{n} d \mu(\lambda)<\infty
$$

and the support of the measure $\mu$,

$$
\operatorname{supp}(\mu)=\{\lambda \in \mathrm{R}: \forall \epsilon>0, \exists \mu((\lambda-\epsilon, \lambda+\epsilon))\}
$$

is an infinite set. Then there exists an infinite sequence of orthogonal polynomials $\left\{p_{n}(\lambda)\right\}$ in the space $\wp$ equipped with scalar product

$$
\left(p_{n}, p_{m}\right)=\int_{\operatorname{supp}(\mu)} p_{n}(\lambda) p_{m}(\lambda) w(\lambda) d \lambda
$$

and the orthogonality relation

$$
\int_{\text {supp }(\mu)} p_{n}(\lambda) p_{m}(\lambda) w(\lambda) d \lambda=h_{n} \delta_{n m},
$$

where $w$ is called weight function. Monic orthogonal polynomials satisfy the three term recurrence relation

$$
\begin{aligned}
\lambda p_{n}(\lambda) & =p_{n+1}(\lambda)+s_{n} p_{n}(\lambda)+r_{n} p_{n-1}(\lambda), \quad n \geq 1 \\
\lambda p_{0}(\lambda) & =p_{1}(\lambda)+s_{0} p_{0}(\lambda),
\end{aligned}
$$

where $s_{n} \in \mathrm{R}$ and $r_{n}>0$. The uniqueness of the measure $\mu$ will be guaranteed when sequences $\left\{s_{n}\right\}$ and $\left\{r_{n}\right\}$ are bounded.

In addition, the orthogonal projection onto $\wp_{N}$ is given by linear map $\Pi_{N}$ : $\wp \rightarrow \wp_{N}$, such that

$$
\Pi_{N} f=\int_{\text {supp }(\mu)} K_{N}\left(\lambda_{i}, \lambda_{j}\right) f\left(\lambda_{j}\right) d \lambda_{j},
$$

where $K_{N}$ is the kernel of the projection operator on the $\mathrm{n}$ dimensional space generated by $\left\{p_{n}(\lambda)\right\}$, and is given by

$$
K_{N}\left(\lambda_{i}, \lambda_{j}\right)=\sqrt{w\left(\lambda_{i}\right) w\left(\lambda_{j}\right)} \sum_{n=0}^{N-1} \frac{p_{n}\left(\lambda_{i}\right) p_{n}\left(\lambda_{j}\right)}{h_{n}},
$$

multiplying and dividing this expression by $\lambda_{i}-\lambda_{j}$, we have

$$
\begin{aligned}
K_{N}\left(\lambda_{i}, \lambda_{j}\right) & =\frac{\sqrt{w\left(\lambda_{i}\right) w\left(\lambda_{j}\right)}}{\lambda_{i}-\lambda_{j}} \sum_{n=0}^{N-1} \frac{\left(\lambda_{i}-\lambda_{j}\right) p_{n}\left(\lambda_{i}\right) p_{n}\left(\lambda_{j}\right)}{h_{n}} \\
K_{N}\left(\lambda_{i}, \lambda_{j}\right) & =\frac{\sqrt{w\left(\lambda_{i}\right) w\left(\lambda_{j}\right)}}{\lambda_{i}-\lambda_{j}} \sum_{n=0}^{N-1} \frac{\left(\lambda_{i} p_{n}\left(\lambda_{i}\right)\right) p_{n}\left(\lambda_{j}\right)-p_{n}\left(\lambda_{i}\right)\left(\lambda_{j} p_{n}\left(\lambda_{j}\right)\right)}{h_{n}} .
\end{aligned}
$$


As $p_{n}$ are monic orthogonal polynomials, then by recurrence relation we find that

$$
\begin{aligned}
\frac{K_{N}\left(\lambda_{i}, \lambda_{j}\right)}{\frac{\sqrt{w\left(\lambda_{i}\right) w\left(\lambda_{j}\right)}}{\lambda_{i}-\lambda_{j}}}= & \sum_{n=0}^{N-1} \frac{\left(p_{n+1}\left(\lambda_{i}\right)+s_{n} p_{n}\left(\lambda_{i}\right)+r_{n} p_{n-1}\left(\lambda_{i}\right)\right) p_{n}\left(\lambda_{j}\right)-}{h_{n}} \\
& \frac{-p_{n}\left(\lambda_{i}\right)\left(p_{n+1}\left(\lambda_{j}\right)+s_{n} p_{n}\left(\lambda_{j}\right)+r_{n} p_{n-1}\left(\lambda_{j}\right)\right)}{h_{n}} \\
= & \sum_{n=0}^{N-1} \frac{p_{n+1}\left(\lambda_{i}\right) p_{n}\left(\lambda_{j}\right)+r_{n} p_{n-1}\left(\lambda_{i}\right) p_{n}\left(\lambda_{j}\right)-p_{n}\left(\lambda_{i}\right) p_{n+1}\left(\lambda_{j}\right)-}{h_{n}} \\
& \frac{-r_{n} p_{n}\left(\lambda_{i}\right) p_{n-1}\left(\lambda_{j}\right)}{h_{n}},
\end{aligned}
$$

one notices that this expression corresponds to a telescoping series, hence simplifies as

$$
\frac{K_{N}\left(\lambda_{i}, \lambda_{j}\right)}{\frac{\sqrt{w\left(\lambda_{i}\right) w\left(\lambda_{j}\right)}}{\lambda_{i}-\lambda_{j}}}=\frac{r_{N} p_{N}\left(\lambda_{i}\right) p_{N-1}\left(\lambda_{j}\right)-r_{N} p_{N-1}\left(\lambda_{i}\right) p_{N}\left(\lambda_{j}\right)}{h_{N}},
$$

using $r_{N}=\frac{h_{N}}{h_{N-1}}$ we obtain

$$
K_{N}\left(\lambda_{i}, \lambda_{j}\right)=\frac{\sqrt{w\left(\lambda_{i}\right) w\left(\lambda_{j}\right)}}{\lambda_{i}-\lambda_{j}} \frac{p_{N}\left(\lambda_{i}\right) p_{N-1}\left(\lambda_{j}\right)-p_{N-1}\left(\lambda_{i}\right) p_{N}\left(\lambda_{j}\right)}{h_{N-1}}
$$

which is the Christoffel-Darboux formula. In consequence the exact form for the density of intervals $[\lambda, \lambda+d \lambda]$ that contains one zero of the orthogonal polynomial is given by

$$
\rho_{N}(\lambda)=\frac{K_{N}(\lambda, \lambda)}{N}=w(\lambda) \frac{p_{N}^{\prime}(\lambda) p_{N-1}(\lambda)-p_{N-1}^{\prime}(\lambda) p_{N}(\lambda)}{h_{N-1}}
$$

All the zeros of the orthogonal polynomials $p_{n}$ are real, simple and lie in the support of the measure $\mu$. Furthermore $p_{n}$ has $n$ distinct zeros in this support. This suggests that in the large $N=n$ limit, the distribution of the zeros of $p_{n}$ converge to the equilibrium distribution.

As all the moments of the orthogonality measure are finite, the Stieltjes transform of this measure for $z \in \mathrm{C} \backslash \mathrm{R}$ is given by

$$
G(z)=\int_{\mathrm{R}} \frac{d \mu(\lambda)}{z-\lambda},
$$

where this transform is a bijective mapping between finite measures and analytic functions on $\mathrm{C} \backslash \mathrm{R}$. In this way, this transform corresponds to a special case of the Cauchy type integrals.

Also since the support of the orthogonality measure is closed, we have that the 
large $\lambda$ limit of this measure is

$$
\lim _{\lambda \rightarrow \infty} d \mu(\lambda)=0
$$

and due to the pole of $G(z)$ at infinity, we can represent $G(z)$ as a generating function of the moments of the orthogonality measure

$$
G(z)=\frac{1}{z}+\frac{\int_{\mathrm{R}} d \mu(\lambda) \lambda^{1}}{z^{2}}+\frac{\int_{\mathrm{R}} d \mu(\lambda) \lambda^{2}}{z^{3}}+\ldots+\frac{\int_{\mathrm{R}} d \mu(\lambda) \lambda^{k}}{z^{k+1}}+\ldots,
$$

where the moments of the orthogonality measure correspond to the generating function for surfaces with one boundary of length $k$ in the planar limit. Indeed,

$$
\int_{\mathrm{R}} d \mu(\lambda) \lambda^{k}=\lim _{N \rightarrow \infty} \frac{\left\langle\operatorname{tr} M^{k}\right\rangle}{N} .
$$

The trace of $M$ can be expressed as function of its eigenvalues $\lambda_{i}$, then we have

$$
\int_{\mathrm{R}} d \mu(\lambda) \lambda^{k}=\lim _{N \rightarrow \infty} \frac{\left\langle\sum_{i} \lambda_{i}^{k}\right\rangle}{N},
$$

where equilibrium measure sustains this equality. Therefore the Stieltjes transform $G(z)$ can be represented as a generating function for surfaces with one boundary of any length, in the following way

$$
\begin{aligned}
G(z) & =\frac{1}{z}+\frac{\lim _{N \rightarrow \infty} \frac{\left\langle\sum_{i} \lambda_{i}\right\rangle}{N}}{z^{2}}+\frac{\lim _{N \rightarrow \infty} \frac{\left\langle\sum_{i} \lambda_{i}^{2}\right\rangle}{N}}{z^{3}}+\ldots+\frac{\lim _{N \rightarrow \infty} \frac{\left\langle\sum_{i} \lambda_{i}^{k}\right\rangle}{N}}{z^{k+1}}+\ldots \\
& =\lim _{N \rightarrow \infty} \frac{1}{N}\left(\left\langle\frac{\sum_{i} 1}{z}\right\rangle+\left\langle\frac{\sum_{i} \lambda_{i}}{z^{2}}\right\rangle+\left\langle\frac{\sum_{i} \lambda_{i}^{2}}{z^{3}}\right\rangle+\ldots+\left\langle\frac{\sum_{i} \lambda_{i}^{k}}{z^{k+1}}\right\rangle+\ldots\right) \\
& =\lim _{N \rightarrow \infty} \frac{1}{N}\left\langle\sum_{i} \frac{1}{z-\lambda_{i}}\right\rangle \\
& =\lim _{N \rightarrow \infty} \frac{1}{N}\left\langle\operatorname{tr} \frac{1}{z-M}\right\rangle \\
G(z) & =\lim _{N \rightarrow \infty} \omega(z) .
\end{aligned}
$$

One notices that Stieltjes transform $G(z)$ yields planar limit of the resolvent function.

Also the Stieltjes transform $G(z)$ can be represented $G(z)$ as a continued fraction [29]

$$
G(z)=\frac{h_{0}}{z+s_{0}-\frac{r_{1}}{z+s_{1}+\frac{r_{2}}{z+s_{2}-\frac{r_{3}}{z+s_{3}-\ldots}}},}
$$


where $s_{n}$ and $r_{n}$ are the sequences of coefficients in the recurrence relation for the orthogonal polynomials.

\section{3}

\section{Analytical Representation}

Since matrix elements of the Van der Monde determinant can be written in terms of orthogonal polynomials

$$
\prod_{i<j}\left(\lambda_{i}-\lambda_{j}\right)=\left|p_{j}\left(\lambda_{i}\right)\right|
$$

recalling that $p_{j}\left(\lambda_{i}\right)$ compounds an infinite ensemble with respect to $e^{-N V(\lambda)} d \lambda$ and by Leibniz formula,

$$
\left|p_{j}\left(\lambda_{i}\right)\right|=\sum_{\sigma \in S_{n}} p_{\sigma(j)}\left(\lambda_{i}\right)(-1)^{\sigma(j)},
$$

the partition function is given by

$$
\begin{aligned}
Z_{N} & =\int\left(\sum_{\sigma \in S_{n}} p_{\sigma(j)}\left(\lambda_{i}\right)(-1)^{\sigma(j)}\right)\left(\sum_{\tau \in S_{n}} p_{\tau(j)}\left(\lambda_{i}\right)(-1)^{\tau(j)}\right) \prod_{i=1}^{N} e^{-N V\left(\lambda_{i}\right)} d \lambda_{i} \\
& =\sum_{\sigma, \tau \in S_{n}}(-1)^{\sigma(j)}(-1)^{\tau(j)} \int p_{\sigma(j)}\left(\lambda_{i}\right) p_{\tau(j)}\left(\lambda_{i}\right) \prod_{i=1}^{N} e^{-N V\left(\lambda_{i}\right)} d \lambda_{i}
\end{aligned}
$$

using the orthogonality relation, we obtain

$$
\begin{aligned}
Z_{N} & =\sum_{\sigma, \tau \in S_{n}}(-1)^{\sigma(i)}(-1)^{\tau(i)} \prod_{i=1}^{N} h_{\sigma(i)} \delta_{\sigma(i) \tau(i)} \\
& =\sum_{\sigma \in S_{n}}(-1)^{\sigma(i)}(-1)^{\sigma(i)} \prod_{i=1}^{N} h_{\sigma(i)} \\
Z_{N} & =\sum_{\sigma \in S_{n}}(1)^{\sigma(i)} \prod_{i=1}^{N} h_{\sigma(i)},
\end{aligned}
$$

since the number of permutations of $N$ indices is $N$ !, we find

$$
Z_{N}=N ! \prod_{i=0}^{N-1} h_{i}
$$

Notice that one can show that the partition function for this model is given by the products of rates, $f_{k}=\frac{h_{k}}{h_{k-1}}$, between the normalizations for the consecutive 
orders, in the following way

$$
\begin{aligned}
Z_{N} & =N !\left(h_{0} h_{1} h_{2} \ldots h_{N-1}\right) \\
& =N !\left[h_{0}\left(\frac{h_{0}^{N-1}}{h_{0}^{N-1}}\right) h_{1}\left(\frac{h_{1}^{N-2}}{h_{1}^{N-2}}\right) h_{2}\left(\frac{h_{2}^{N-3}}{h_{2}^{N-3}}\right) h_{3} \ldots\left(\frac{h_{N-2}}{h_{N-2}}\right) h_{N-1}\right] \\
& =N !\left[h_{0}^{N}\left(\frac{h_{1}^{N-1}}{h_{0}^{N-1}}\right)\left(\frac{h_{2}^{N-2}}{h_{1}^{N-2}}\right)\left(\frac{h_{3}^{N-3}}{h_{2}^{N-3}}\right) \ldots\left(\frac{h_{N-1}}{h_{N-2}}\right)\right] \\
& =N ! h_{0}^{N} \prod_{k=1}^{N-1} f_{k}^{N-k}
\end{aligned}
$$

then the free energy of the model is given by

$$
F=\log Z_{N}=\log (N !)+N \log h_{0}+N \sum_{k}\left(1-\frac{k}{N}\right) \log f_{k} .
$$

To understand the role or meaning of $f_{n}$, let us look it

$$
h_{n}=\int d \lambda e^{-N V(\lambda)} p_{n}(\lambda)\left(\lambda p_{n-1}(\lambda)\right)
$$

by the three term recurrence relation, it reads

$$
\begin{aligned}
h_{n} & =\int d \lambda e^{-N V(\lambda)}\left(p_{n+1}(\lambda)+r_{n} p_{n-1}(\lambda)\right) p_{n-1}(\lambda) \\
& =\int d \lambda e^{-N V(\lambda)} p_{n+1}(\lambda) p_{n-1}(\lambda)+r_{n} \int d \lambda e^{-N V(\lambda)} p_{n-1}(\lambda) p_{n-1}(\lambda) \\
h_{n} & =r_{n} h_{n-1}
\end{aligned}
$$

as has been noted using the definition of $f_{n}$, we obtain

$$
r_{n}=\frac{h_{n}}{h_{n-1}}=f_{n}
$$

notice that the sufficient condition for computing the free energy, and in consecuence the correlation functions is the coeficient $r_{n}$ of the recurrence relation.

On the other hand, the trick to find one relation between the potential $V$ and the normalization constants consists in evaluating the following integral

$$
\begin{aligned}
\int d \lambda e^{-N V(\lambda)} p_{n}^{\prime}(\lambda) p_{n-1}(\lambda)= & \int d \lambda e^{-N V(\lambda)}\left(n p_{n-1}(\lambda)+O\left(\lambda^{n-2}\right)\right) p_{n-1}(\lambda) \\
= & \int d \lambda e^{-N V(\lambda)} n p_{n-1}(\lambda) p_{n-1}(\lambda)+ \\
& +\int d \lambda e^{-N V(\lambda)} O\left(\lambda^{n-2}\right) p_{n-1}(\lambda) \\
= & n h_{n-1}
\end{aligned}
$$


and alternativaly using integration by parts we obtain

$$
\begin{aligned}
\int d \lambda e^{-N V(\lambda)} p_{n}^{\prime}(\lambda) p_{n-1}(\lambda)= & {\left[\prod_{i=1}^{N} e^{-N V(\lambda)} p_{n}\left(\lambda_{i}\right) p_{n-1}\left(\lambda_{i}\right)\right]_{-\infty}^{\infty}-} \\
& -\int d \lambda p_{n}(\lambda) \frac{d}{d x}\left(e^{-N V(\lambda)} p_{n-1}(\lambda)\right) \\
= & -\int d \lambda p_{n}(\lambda)\left(-N V^{\prime}(\lambda) e^{-N V(\lambda)} p_{n-1}(\lambda)+\right. \\
& \left.+e^{-N V(\lambda)}(n-1) p_{n-2}(\lambda)+e^{-N V(\lambda)} O\left(\lambda^{n-3}\right)\right) \\
= & \int d \lambda e^{-N V(\lambda)} p_{n}(\lambda)\left(N V^{\prime}(\lambda)\right) p_{n-1}(\lambda)- \\
& -(n-1) \int d \lambda e^{-N V(\lambda)} p_{n}(\lambda) p_{n-2}(\lambda)- \\
& \left.-\int d \lambda e^{-N V(\lambda)} p_{n}(\lambda) O\left(\lambda^{n-3}\right)\right) \\
= & \int d \lambda e^{-N V(\lambda)} N V^{\prime}(\lambda) p_{n}(\lambda) p_{n-1}(\lambda)
\end{aligned}
$$

and finally comparing both results $(4.27),(4.28)$ we have the relation

$$
n h_{n-1}=\int d \lambda e^{-N V(\lambda)} N V^{\prime}(\lambda) p_{n}(\lambda) p_{n-1}(\lambda)
$$

which is called string equation.

For example, in the GUE, we have the quadratic potential, $V(\lambda)=\frac{1}{2} \lambda^{2}$. Hence the measure in the corresponding partition function

$$
Z_{N}=C_{N} \int d \lambda e^{-\frac{N}{2} \lambda^{2}} p_{i}(\lambda) p_{j}(\lambda)
$$

allow expressing this partition function with Hermite polynomials,

$$
p_{i}(\lambda)=\left(\frac{1}{2 N}\right)^{\frac{i}{2}} H_{i}\left(\sqrt{\frac{N}{2}} \lambda\right)
$$

such that inserting and integrating this polynomial in the orthogonality relation we find the normalization constants given by

$$
h_{i}=\frac{1}{\sqrt{2 \pi N}} i ! N^{-i} \text {. }
$$

Thus the coefficient $r_{i}$ of the equation (4.26),

$$
r_{i}=\frac{h_{i}}{h_{i-1}}=\frac{i}{N},
$$


agrees to the following recurrence relation,

$$
\lambda p_{i}(\lambda)=p_{i+1}(\lambda)+\frac{i}{N} p_{i-1}(\lambda)
$$

In this way the partition function by the equation (4.22) is given by

$$
Z_{N}=N !\left(\frac{1}{\sqrt{2 \pi N}}\right)^{N} \prod_{i=1}^{N-1}\left(\frac{i}{N}\right)^{N-i}
$$

and the free energy corresponding to the equation (4.23) is given by

$$
F=\log Z_{N}=\log (N !)+N \log \left(\frac{1}{\sqrt{2 \pi N}}\right)+N \sum_{i}\left(1-\frac{i}{N}\right) \log \left(\frac{i}{N}\right) .
$$

\section{4}

\section{Geometrical Representation}

String equation can be interpreted as staircase path, because involves terms of the form

$$
\int d \lambda_{i} e^{-V\left(\lambda_{i}\right)} p_{n}\left(\lambda_{i}\right) \lambda^{2 p+1} p_{n-1}\left(\lambda_{i}\right)
$$

For general even potentials

$$
V(\lambda)=\frac{\lambda^{2}}{2}+\sum_{p>1} \frac{g_{2 p}}{2 p} \lambda^{2 p}
$$

hence,

$$
V^{\prime}(\lambda)=\lambda+\sum_{p>1} g_{2 p} \lambda^{2 p-1}
$$

computing the right side in the string equation

$$
\begin{aligned}
\int d \lambda_{i} e^{-V\left(\lambda_{i}\right)} p_{n}\left(\lambda_{i}\right) V^{\prime}(\lambda) p_{n-1}\left(\lambda_{i}\right)= & \int d \lambda_{i} e^{-V\left(\lambda_{i}\right)} p_{n}\left(\lambda_{i}\right)\left(\lambda+\sum_{p>1} g_{2 p} \lambda^{2 p-1}\right) \times \\
& \times p_{n-1}\left(\lambda_{i}\right) \\
= & \int d \lambda_{i} e^{-V\left(\lambda_{i}\right)} p_{n}\left(\lambda_{i}\right) \lambda p_{n-1}\left(\lambda_{i}\right)+ \\
& \sum_{p>1} g_{2 p} \int d \lambda_{i} e^{-V\left(\lambda_{i}\right)} p_{n}\left(\lambda_{i}\right) \lambda^{2 p-1} p_{n-1}\left(\lambda_{i}\right) \\
= & h_{n}+\sum_{p>0} g_{2 p+2} \int d \lambda_{i} e^{-V\left(\lambda_{i}\right)} p_{n}\left(\lambda_{i}\right) \lambda^{2 p+1} \\
& \times p_{n-1}\left(\lambda_{i}\right) \\
= & h_{n}+\sum_{p>0} g_{2 p+2} \alpha_{n}^{[2 p+1]},
\end{aligned}
$$


where $\alpha_{n}^{[2 p+1]}$ is given by

$$
\alpha_{n}^{[2 p+1]}=\int d \lambda_{i} e^{-V\left(\lambda_{i}\right)} p_{n}\left(\lambda_{i}\right) \lambda^{2 p+1} p_{n-1}\left(\lambda_{i}\right)
$$

And since $p_{n}$ form a complete set of basis vector in the espace of polynomials, one can identify $\alpha_{n}^{[2 p+1]}$ as a coefficient of the linear expansion of $\lambda^{2 p+1} p_{n-1}\left(\lambda_{i}\right)$,

$$
\lambda^{2 p+1} p_{n-1}\left(\lambda_{i}\right)=\sum_{n} \alpha_{n}^{[2 p+1]} p_{n}\left(\lambda_{i}\right) .
$$

Analogously, for the next order in $\lambda$,

$$
\lambda^{2 p+2} p_{n-1}\left(\lambda_{i}\right)=\sum_{m} \alpha_{m}^{[2 p+2]} p_{m}\left(\lambda_{i}\right)
$$

moreover, this expansion can be expressed in terms of $p_{n}$,

$$
\lambda^{2 p+2} p_{n-1}\left(\lambda_{i}\right)=\lambda^{2 p+1}\left(\lambda_{i} p_{n-1}\left(\lambda_{i}\right)\right)=\sum_{n} \alpha_{n}^{[2 p+1]}\left(\lambda_{i} p_{n}\left(\lambda_{i}\right)\right),
$$

using the recursion relation for $p_{n}$ and comparing with the equation (4.34), we obtain

$$
\begin{aligned}
\sum_{m} \alpha_{m}^{[2 p+2]} p_{m}\left(\lambda_{i}\right) & =\sum_{n} \alpha_{n}^{[2 p+1]}\left(p_{n+1}\left(\lambda_{i}\right)+r_{n} p_{n-1}\left(\lambda_{i}\right)\right) \\
& =\sum_{n+1} \alpha_{n-1}^{[2 p+1]} p_{n}\left(\lambda_{i}\right)+\sum_{n-1} r_{n+1} \alpha_{n+1}^{[2 p+1]} p_{n}\left(\lambda_{i}\right)
\end{aligned}
$$

For fixed $m=n$, the above identity leads to a recursion relation for the coeficients, $\alpha_{m}^{[2 p+2]}$, given by

$$
\alpha_{n}^{[2 p+2]}=\alpha_{n-1}^{[2 p+1]}+r_{n+1} \alpha_{n+1}^{[2 p+1]}
$$

moreover in the equation (4.33) for $2 p+1=0$, by uniqueness of the orthogonal polynomials we get the initial condition to the recurrence relation,

$$
\alpha_{n}^{[0]}=\delta_{n, n-1} .
$$

One way of representing this recurrence relation, is as all possible staircase walk of $2 p+1$ steps that joining height $n-1$ to height $n$ such that for each step we attach a weight 1 for a step up or weight $r_{l}$ for step down when we leave height $l$ and the total contribution is the product of all this weights. Therefore $\alpha_{n}^{[2 p+1]}$ is a sum over the $\left(\begin{array}{c}2 p+1 \\ p\end{array}\right)$ paths along the staircase, with $p$ steps up and $p-1$ steps down.

For example when $p=1$, we have $\left(\begin{array}{l}3 \\ 1\end{array}\right)=3$ paths, represented in Figure 


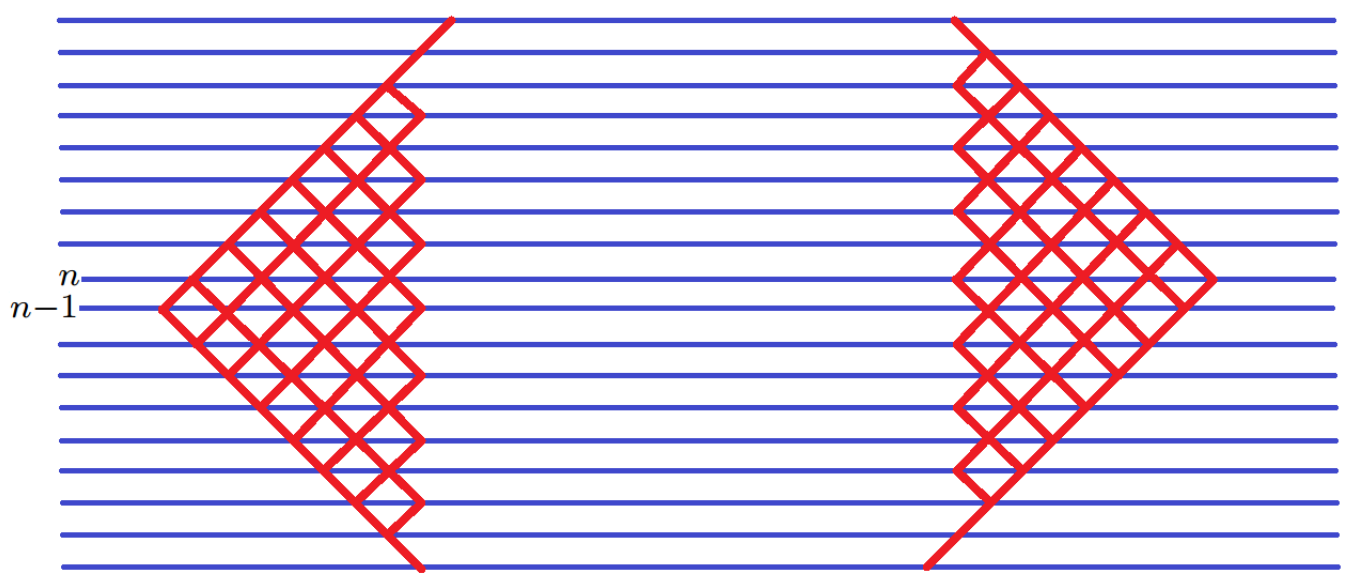

Figure 4.1: All possibles staircase walks connecting height $n-1$ to height $n$ for $\alpha_{n}^{[2 p+1]}$.

\subsection{We can write}

$$
\alpha_{n}^{[3]}=1 \times 1 \times r_{n+1}+1 \times r_{n} \times 1+r_{n-1} \times 1 \times 1=r_{n+1}+r_{n}+r_{n-1} .
$$

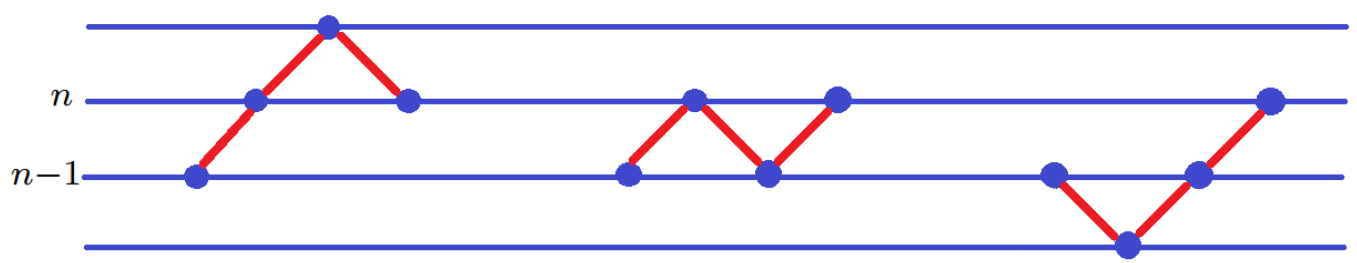

Figure 4.2: Staircase walks corresponding to $p=1$.

\section{5}

\section{Algebraic Representation}

In view of $\left\{p_{n}(\lambda)\right\}$ form a basis in the linear space of polynomials $\wp$, we can define two operators $Q$ and $P$ that acting on this basis, such that one multiplies the polynomial by $\lambda$ and the other take the derivative of the polynomial with respect to $\lambda$ respectivaly. So that one can represent this operators as matrices in the following way

$$
\begin{aligned}
\lambda p_{n}(\lambda) & =\sum_{m} Q_{n m} p_{m}(\lambda) \\
\frac{d}{d \lambda} p_{n}(\lambda) & =\sum_{m} P_{n m} p_{m}(\lambda) .
\end{aligned}
$$


In fact, the matrix elements of $Q$ are given by the scalar product

$$
\begin{aligned}
Q_{n m} & =\left(p_{n}, Q p_{m}\right) \\
& =\left(p_{n}, \lambda p_{m}\right),
\end{aligned}
$$

using the three term recurrence relation, we have

$$
Q_{n m}=\left(p_{n}, p_{m+1}+s_{m} p_{m}+r_{m} p_{m-1}\right)
$$

due to the orthogonality relation, $\left(p_{n}, p_{m}\right)=h_{n} \delta_{n m}$, we obtain

$$
\begin{aligned}
& Q_{n m}=\left(p_{n}, p_{m+1}\right)+s_{m}\left(p_{n}, p_{m}\right)+r_{m}\left(p_{n}, p_{m-1}\right) \\
& Q_{n m}=h_{n} \delta_{n, m+1}+s_{m} h_{n} \delta_{n m}+r_{m} h_{n} \delta_{n, m-1},
\end{aligned}
$$

consequently the matrix $\mathrm{Q}$ is given by

$$
Q=\left(\begin{array}{cccccc}
s_{0} h_{0} & h_{1} & 0 & 0 & 0 & \cdots \\
r_{1} h_{0} & s_{1} h_{1} & h_{2} & 0 & 0 & \cdots \\
0 & r_{2} h_{1} & s_{2} h_{2} & h_{3} & 0 & \cdots \\
0 & 0 & r_{3} h_{2} & s_{3} h_{3} & h_{4} & \cdots \\
\vdots & \vdots & \vdots & \vdots & \vdots & \ddots
\end{array}\right),
$$

notice that this matrix is tridiagonal and is called Jacobi matrix. But it is not symmetric because this characteristic depends on the basis. For instance, one can obtain a symmetric Jacobi matrix in the basis $\left\{\psi_{n}(\lambda)=\frac{p_{n}(\lambda) e^{\frac{-N V(\lambda)}{2}}}{\sqrt{h_{n}}}\right\}$. Identically, the matrix elements of $P$ are defined of the following way

$$
\begin{aligned}
P_{n m} & =\left(p_{n}, P p_{m}\right) \\
& =\left(p_{n}, \frac{d}{d \lambda} p_{m}\right) \\
& =\int_{-\infty}^{\infty} e^{-N V(\lambda)} d \lambda p_{n}(\lambda) \frac{d p_{m}(\lambda)}{d \lambda},
\end{aligned}
$$

using integration by parts, we have

$$
\begin{aligned}
P_{n m} & =-\int_{-\infty}^{\infty} p_{m}(\lambda) \frac{d}{d \lambda}\left(e^{-N V(\lambda)} p_{n}(\lambda)\right) \\
& =-\int_{-\infty}^{\infty} e^{-N V(\lambda)} p_{m}(\lambda)\left(-N V^{\prime}(\lambda)\right) p_{n}(\lambda)-\int_{-\infty}^{\infty} e^{-N V(\lambda)} p_{m}(\lambda) \frac{d}{d \lambda}\left(p_{n}(\lambda)\right) \\
& =\left(p_{m}, N V^{\prime}(\lambda) p_{n}\right)-\left(p_{m}, \frac{d}{d \lambda} p_{n}\right),
\end{aligned}
$$


using the operator $Q$, we obtain

$$
\begin{aligned}
P_{n m} & =N\left(p_{n}, V^{\prime}(Q) p_{m}\right)-\left(p_{m}, P p_{n}\right) \\
P_{n m} & =N\left[V^{\prime}(Q)\right]_{n m}-P_{m n} .
\end{aligned}
$$

Since, $\frac{d}{d \lambda} p_{n}(\lambda)=n p_{n-1}(\lambda)+O\left(\lambda^{n-2}\right)$, we have that

$$
n p_{n-1}(\lambda)+O\left(\lambda^{n-2}\right)=\sum_{m>n-1} P_{n m} p_{m}(\lambda)
$$

Notice that the matrix $P$ is lower triangular. In this way, if $P_{n m}$ is lower triangular, then $P_{m n}$ corresponds to upper triangular. Hence by the equation (4.45), we obtain

$$
P_{n m}=N\left[V^{\prime}(Q)_{-}\right]_{n m},
$$

where - denote the lower triangular part of $V^{\prime}(Q)$.

In fact, the commutation relation of $Q$ and $P$ in the basis $\left\{p_{n}(\lambda)\right\}$ is given by

$$
\begin{aligned}
\sum_{m}[P, Q]_{n m} p_{m}(\lambda) & =\sum_{m}(P Q-Q P)_{n m} p_{m}(\lambda) \\
& =\sum_{l, m}\left(P_{n l} Q_{l m} p_{m}(\lambda)-Q_{n l} P_{l m} p_{m}(\lambda)\right) \\
& =\sum_{l}\left(P_{n l} \sum_{m} Q_{l m} p_{m}(\lambda)-Q_{n l} \sum_{m} P_{l m} p_{m}(\lambda)\right) \\
& =\sum_{l}\left(P_{n l} \lambda p_{l}(\lambda)-Q_{n l} \frac{d}{d \lambda} p_{l}(\lambda)\right) \\
& =\frac{d}{d \lambda}\left(\lambda p_{n}(\lambda)\right)-\lambda \frac{d}{d \lambda} p_{n}(\lambda) \\
& =p_{n}(\lambda)+\lambda \frac{d}{d \lambda} p_{n}(\lambda)-\lambda \frac{d}{d \lambda} p_{n}(\lambda) \\
\sum_{m}[P, Q]_{n m} p_{m}(\lambda) & =p_{n}(\lambda),
\end{aligned}
$$

here $[P, Q]_{n m}=\delta_{n m}$, therefore

$$
[P, Q]=1
$$

\section{6}

\section{Statistical Representation}

Recall that the orthogonal polynomials form an infinite set with respect to the measure

The Van der Monde determinant is given by

$$
\int d \lambda_{i} e^{-N V\left(\lambda_{i}\right)} p_{n}\left(\lambda_{i}\right) p_{m}\left(\lambda_{i}\right)=h_{n} \delta_{n m} .
$$




$$
\prod_{i<j}\left(\lambda_{i}-\lambda_{j}\right)=(-1)^{\frac{N(N-1)}{2}}\left|\begin{array}{ccccc}
1 & 1 & 1 & \cdots & 1 \\
\lambda_{1} & \lambda_{2} & \lambda_{3} & \cdots & \lambda_{N} \\
\vdots & \vdots & \vdots & \ddots & \vdots \\
\lambda_{1}^{N-1} & \lambda_{2}^{N-1} & \lambda_{3}^{N-1} & \cdots & \lambda_{N}^{N-1}
\end{array}\right|
$$

Now by multilinearity of the determinant, we add up the first row to the second row of the Van der Monde determinant, and replace the second row with $\left(p_{1}\left(\lambda_{1}\right), p_{1}\left(\lambda_{2}\right), p_{1}\left(\lambda_{3}\right), \cdots, p_{1}\left(\lambda_{N}\right)\right)$ without changing the determinant. Hence by adding up appropriate combinations of the first rows to the third row, and replace the third row with $\left(p_{2}\left(\lambda_{1}\right), p_{2}\left(\lambda_{2}\right), p_{2}\left(\lambda_{3}\right), \cdots, p_{2}\left(\lambda_{N}\right)\right)$ without changing the determinant. Thus doing the same in the others rows, we have

$$
\prod_{i<j}\left(\lambda_{i}-\lambda_{j}\right)=(-1)^{\frac{N(N-1)}{2}}\left|\begin{array}{cccc}
p_{0}\left(\lambda_{1}\right) & p_{0}\left(\lambda_{2}\right) & \cdots & p_{0}\left(\lambda_{N}\right) \\
p_{1}\left(\lambda_{1}\right) & p_{1}\left(\lambda_{2}\right) & \cdots & p_{1}\left(\lambda_{N}\right) \\
\vdots & \vdots & \ddots & \vdots \\
p_{N-1}\left(\lambda_{1}\right) & p_{N-1}\left(\lambda_{2}\right) & \cdots & p_{N-1}\left(\lambda_{N}\right)
\end{array}\right|
$$

hence, by $|M|=\left|M^{T}\right|$, we obtain

$$
\begin{aligned}
\prod_{i<j}\left(\lambda_{i}-\lambda_{j}\right)^{2}= & (-1)^{N(N-1)}\left|\begin{array}{ccc}
p_{0}\left(\lambda_{1}\right) & \cdots & p_{N-1}\left(\lambda_{1}\right) \\
p_{0}\left(\lambda_{2}\right) & \cdots & p_{N-1}\left(\lambda_{2}\right) \\
\vdots & \ddots & \vdots \\
p_{0}\left(\lambda_{N}\right) & \cdots & p_{N-1}\left(\lambda_{N}\right)
\end{array}\right| \times \\
& \times\left|\begin{array}{ccc}
p_{0}\left(\lambda_{1}\right) & \cdots & p_{0}\left(\lambda_{N}\right) \\
p_{1}\left(\lambda_{1}\right) & \cdots & p_{1}\left(\lambda_{N}\right) \\
\vdots & \ddots & \vdots \\
p_{N-1}\left(\lambda_{1}\right) & \cdots & p_{N-1}\left(\lambda_{N}\right)
\end{array}\right|
\end{aligned}
$$

And the $j p d f$ of the eigenvalues can be expressed as

$$
\begin{aligned}
d \mu_{N}(\lambda)= & \frac{1}{Z_{N}}\left|\begin{array}{ccc}
p_{0}\left(\lambda_{1}\right) & \cdots & p_{N-1}\left(\lambda_{1}\right) \\
p_{0}\left(\lambda_{2}\right) & \cdots & p_{N-1}\left(\lambda_{2}\right) \\
\vdots & \ddots & \vdots \\
p_{0}\left(\lambda_{N}\right) & \cdots & p_{N-1}\left(\lambda_{N}\right)
\end{array}\right|\left|\begin{array}{ccc}
p_{0}\left(\lambda_{1}\right) & \cdots & p_{0}\left(\lambda_{N}\right) \\
p_{1}\left(\lambda_{1}\right) & \cdots & p_{1}\left(\lambda_{N}\right) \\
\vdots & \ddots & \vdots \\
p_{N-1}\left(\lambda_{1}\right) & \cdots & p_{N-1}\left(\lambda_{N}\right)
\end{array}\right| \times \\
& \times \Pi_{i=1}^{N} e^{-N V\left(\lambda_{i}\right)} d \lambda_{i},
\end{aligned}
$$


multiplying by $e^{-\frac{N}{2} V\left(\lambda_{i}\right)}$ to each row $i$ in the first determinant and to each column $i$ in the second determinant, we get

$$
\begin{aligned}
& d \mu_{N}(\lambda)=\frac{d \lambda}{Z_{N}}\left|\begin{array}{ccc}
e^{\frac{-N V\left(\lambda_{1}\right)}{2}} p_{0}\left(\lambda_{1}\right) & \cdot & e^{\frac{-N V\left(\lambda_{1}\right)}{2}} p_{N-1}\left(\lambda_{1}\right) \\
e^{\frac{-N V\left(\lambda_{2}\right)}{2}} p_{0}\left(\lambda_{2}\right) & \cdot & e^{\frac{-N V\left(\lambda_{2}\right)}{2}} p_{N-1}\left(\lambda_{2}\right) \\
\vdots & \cdot & \vdots \\
e^{\frac{-N V\left(\lambda_{N}\right)}{2}} p_{0}\left(\lambda_{N}\right) & \cdot & e^{\frac{-N V\left(\lambda_{N}\right)}{2}} p_{N-1}\left(\lambda_{N}\right)
\end{array}\right| \\
& \times\left|\begin{array}{ccc}
e^{\frac{-N V\left(\lambda_{1}\right)}{2}} p_{0}\left(\lambda_{1}\right) & \cdot & e^{\frac{-N V\left(\lambda_{N}\right)}{2}} p_{0}\left(\lambda_{N}\right) \\
e^{\frac{-N V\left(\lambda_{1}\right)}{2}} p_{1}\left(\lambda_{1}\right) & \cdot & e^{\frac{-N V\left(\lambda_{N}\right)}{2}} p_{1}\left(\lambda_{N}\right) \\
\vdots & \cdot & \vdots \\
e^{\frac{-N V\left(\lambda_{1}\right)}{2}} p_{N-1}\left(\lambda_{1}\right) & \cdot & e^{\frac{-N V\left(\lambda_{N}\right)}{2}} p_{N-1}\left(\lambda_{N}\right)
\end{array}\right|,
\end{aligned}
$$

resulting as expected in

$$
d \mu_{N}(\lambda)=\frac{d \lambda}{Z_{N}}\left|\begin{array}{cc}
e^{\frac{-N\left(V\left(\lambda_{1}\right)+V\left(\lambda_{1}\right)\right)}{2}} \sum_{n} p_{n}\left(\lambda_{1}\right) p_{n}\left(\lambda_{1}\right) & \cdots \\
e^{\frac{-N\left(V\left(\lambda_{2}\right)+V\left(\lambda_{1}\right)\right)}{2}} \sum_{n} p_{n}\left(\lambda_{2}\right) p_{n}\left(\lambda_{1}\right) & \ldots \\
\vdots & \ddots \\
e^{\frac{-N\left(V\left(\lambda_{N-1}\right)+V\left(\lambda_{1}\right)\right)}{2}} \sum_{n} p_{n}\left(\lambda_{N-1}\right) p_{n}\left(\lambda_{1}\right) & \cdots
\end{array}\right| .
$$

Denoting each matrix element as $K_{N}\left(\lambda_{i}, \lambda_{j}\right)=e^{\frac{-N\left(V\left(\lambda_{i}\right)+V\left(\lambda_{j}\right)\right)}{2}} \sum_{n} \frac{1}{h_{n}} p_{n}\left(\lambda_{i}\right) p_{n}\left(\lambda_{j}\right)$, we obtain

$$
d \mu_{N}(\lambda)=d \lambda\left|\begin{array}{ccc}
K_{N}\left(\lambda_{1}, \lambda_{1}\right) & \cdots & K_{N}\left(\lambda_{1}, \lambda_{N-1}\right) \\
K_{N}\left(\lambda_{2}, \lambda_{1}\right) & \cdots & K_{N}\left(\lambda_{2}, \lambda_{N-1}\right) \\
\vdots & \ddots & \vdots \\
K_{N}\left(\lambda_{N-1}, \lambda_{1}\right) & \cdots & K_{N}\left(\lambda_{N-1}, \lambda_{N-1}\right)
\end{array}\right| .
$$

Now since the determinant is invariant under permutations $\left(\lambda_{\sigma(1)}, \lambda_{\sigma(2)}, \ldots, \lambda_{\sigma(N)}\right)$, where $\sigma$ is any permutation of $\{1,2, \ldots, N\}$, we have

$$
\begin{aligned}
& d \mu_{N}(\lambda)=d \lambda\left|\begin{array}{ccc}
K_{N}\left(\lambda_{\sigma(1)}, \lambda_{\sigma(1)}\right) & \cdots & K_{N}\left(\lambda_{\sigma(1)}, \lambda_{\sigma(N)}\right) \\
K_{N}\left(\lambda_{\sigma(2)}, \lambda_{\sigma(1)}\right) & \cdots & K_{N}\left(\lambda_{\sigma(2)}, \lambda_{\sigma(N)}\right) \\
\vdots & \ddots & \vdots \\
K_{N}\left(\lambda_{\sigma(N)}, \lambda_{\sigma(1)}\right) & \cdots & K_{N}\left(\lambda_{\sigma(N)}, \lambda_{\sigma(N)}\right)
\end{array}\right| \\
& =\frac{d \lambda}{N !}\left|\begin{array}{ccc}
K_{N}\left(\lambda_{1}, \lambda_{1}\right) & \cdots & K_{N}\left(\lambda_{1}, \lambda_{N-1}\right) \\
K_{N}\left(\lambda_{2}, \lambda_{1}\right) & \cdots & K_{N}\left(\lambda_{2}, \lambda_{N-1}\right) \\
\vdots & \ddots & \vdots \\
K_{N}\left(\lambda_{N-1}, \lambda_{1}\right) & \cdots & K_{N}\left(\lambda_{N-1}, \lambda_{N-1}\right)
\end{array}\right| .
\end{aligned}
$$


Finally, by using $\Pi_{i<j}\left(\lambda_{j}-\lambda_{i}\right)=(-1)^{\frac{N(N-1)}{2}} \operatorname{det}\left(\lambda_{j}^{i}\right)=(-1)^{\frac{N(N-1)}{2}} \operatorname{det} p_{i}\left(\lambda_{j}\right)$, the eigenvalue $j p d f$ reads

$$
d \mu\left(\lambda_{1}, \ldots, \lambda_{N}\right)=\frac{1}{N !} \operatorname{det}\left(K_{N}\left(\lambda_{i}, \lambda_{j}\right)\right)_{i, j=1}^{N},
$$

where the kernel, $K_{N}\left(\lambda_{i}, \lambda_{j}\right)=e^{-\frac{1}{2} N\left(V\left(\lambda_{i}\right)+V\left(\lambda_{j}\right)\right)} \sum_{n=0}^{N-1} \frac{1}{h_{n}} p_{n}\left(\lambda_{i}\right) p_{n}\left(\lambda_{j}\right)$, has the following properties

$$
\begin{aligned}
\int K_{N}\left(\lambda_{i}, \lambda_{k}\right) K_{N}\left(\lambda_{k}, \lambda_{j}\right) d \lambda_{k} & =K_{N}\left(\lambda_{i}, \lambda_{j}\right) \\
\int K_{N}\left(\lambda_{i}, \lambda_{i}\right) d \lambda_{i} & =N
\end{aligned}
$$

such that by this properties one can obtain the following relation

$$
\int \operatorname{det}\left(K_{N}\left(\lambda_{i}, \lambda_{j}\right)\right)_{i, j=1}^{k+1} d \lambda_{k+1}=(N-k) \operatorname{det}\left(K_{N}\left(\lambda_{i}, \lambda_{j}\right)\right)_{i, j=1}^{k} .
$$

If we use this relation to integrate the eigenvalue $j p d f$ over all eigenvalues except $\lambda_{1}, \ldots, \lambda_{n}$, then the $n$-point correlation function $R_{n}$ is given as

$$
R_{n}\left(\lambda_{1}, \ldots, \lambda_{n}\right)=\frac{N !}{(N-n) !} \int d \mu_{N}(\lambda) d \lambda_{n+1} \ldots d \lambda_{N}
$$

by the relation between the $j p d f$ and the kernel we can write

$$
\begin{aligned}
R_{n}\left(\lambda_{1}, \ldots, \lambda_{n}\right) & =\frac{N !}{(N-n) !} \int\left(\frac{1}{N !} \operatorname{det}\left(K_{N}\left(\lambda_{i}, \lambda_{j}\right)\right)_{i, j=1}^{N}\right) d \lambda_{n+1} \ldots d \lambda_{N} \\
& =\frac{1}{(N-n) !} \int \operatorname{det}\left(K_{N}\left(\lambda_{i}, \lambda_{j}\right)\right)_{i, j=1}^{N} d \lambda_{n+1} \ldots d \lambda_{N},
\end{aligned}
$$

integrating over $\lambda_{N}$ we have by the relation (4.56),

$R_{n}\left(\lambda_{1}, \ldots, \lambda_{n}\right)=\frac{1}{(N-n) !} \int(N-(N-1)) \operatorname{det}\left(K_{N}\left(\lambda_{i}, \lambda_{j}\right)\right)_{i, j=1}^{N-1} d \lambda_{n+1} \ldots d \lambda_{N-1}$.

Now integrating over $\lambda_{N-1}$ in the same way we obtain

$R_{n}\left(\lambda_{1}, \ldots, \lambda_{n}\right)=\frac{1}{(N-n) !} \int 1 \times(N-(N-2)) \operatorname{det}\left(K_{N}\left(\lambda_{i}, \lambda_{j}\right)\right)_{i, j=1}^{N-2} d \lambda_{n+1} \ldots d \lambda_{N-2}$

hence performing the step repeatedly

$$
R_{n}\left(\lambda_{1}, \ldots, \lambda_{n}\right)=\operatorname{det}\left(K_{N}\left(\lambda_{i}, \lambda_{j}\right)\right)_{i, j=1}^{n} .
$$

The 1-point correlation function yields the eigenvalue density,

$$
\rho(\lambda)=\lim _{N \rightarrow \infty} \frac{1}{N} R_{1}(\lambda)=\lim _{N \rightarrow \infty} \frac{1}{N} K_{N}(\lambda, \lambda) .
$$

One observes that all the statistical information of the eigenvalue distribution is contained in the correlation kernel. This correlation can be expressed only 
in terms of $p_{N-1}$ and $p_{N}$ due to the Christoffel-Darboux formula

$$
K_{N}\left(\lambda_{i}, \lambda_{j}\right)=e^{-\frac{1}{2} N\left(V\left(\lambda_{i}\right)+V\left(\lambda_{j}\right)\right)} \frac{1}{h_{N-1}} \frac{p_{N}\left(\lambda_{i}\right) p_{N-1}\left(\lambda_{j}\right)-p_{N-1}\left(\lambda_{i}\right) p_{N}\left(\lambda_{j}\right)}{\lambda_{i}-\lambda_{j}}
$$

which can be described by a Riemann Hilbert problem. 


\section{5 \\ Riemann Hilbert problem}

A Riemann Hilbert problem (RHP) is a powerful method in asymptotic analysis of differential and integral equations with applications in many areas, in particular such as orthogonal polynomials and random matrix theory (RMT). In this chapter we introduce basic notions of complex analysis such as the theory of Cauchy type integrals. We discuss the scalar RHP. This discussion is generalized to matrix RHP such that in some cases the matrix RHP can be reduced to the analysis of an array of scalar RHPs. When this is not possible the analysis is given by singular integral equations. Furthermore we review the relation of orthogonal polynomials in RMT and RHPs. In the first part of this chapter we follow the exposure of [2], whereas the second part is based on the text [13].

\section{1}

\section{Introduction}

In physics, a fundamental question of integrability is that the local properties of an object yield complete information about its global behavior. Similarly in complex analysis a complex function can be reconstructed from the known structure of its singularities. In turn, both questions almost always can be formulated as a Riemann Hilbert problem.

In an analogous way, a lot of non linear differential equations can be reduced to RHPs. This differential equations arose from physical applications, promoting the development of the Riemann Hilbert theory. Among other things, RHP are also related to other kinds of equations, for example

1. Find a function, $w(z)=a(x, y)+i b(x, y)$, analytic inside a contour $S$, such that its components on this contour satisfy

$$
\alpha(t) a(t)+\beta(t) b(t)=\gamma(t), \quad t \in S
$$

2. Linear integral equations, by knowing integrable functions $\alpha$ and $\beta$, find a function $f$ that satisfy

$$
f(t)+\int_{0}^{\infty} \alpha\left(t-t^{\prime}\right) f\left(t^{\prime}\right) d t^{\prime}=\beta(t), \quad t>0
$$


3. Reconstruct a function $q(x)$ from appropriate scattering data in the timeindependent Schrödinger equation

$$
\Psi_{x x}+\left(q(x)+k^{2}\right) \Psi=0, \quad-\infty<x<\infty
$$

This problem is also solved by inverse scattering method and has many areas of applications, from geophysics to quantum theory.

Problem 1 was formulated by Riemann in 1851. In 1904, Hilbert reduced this problem to a RHP. Problem 2 can be converted to scalar RHP, while problem 3 is associated with matrix RHP, which its formulation is similar to that for scalar ones.

The essential idea behind a Riemann Hilbert problem is finding an analytic function in the complex plane with a known jump across a curve. More precisely, consider a curve $\gamma$, which can be a simple closed curve, smooth arc, contour or finite union of arcs and contours that intersect at finite number of points.

The orientation of the curve defines the positive side on the left, while

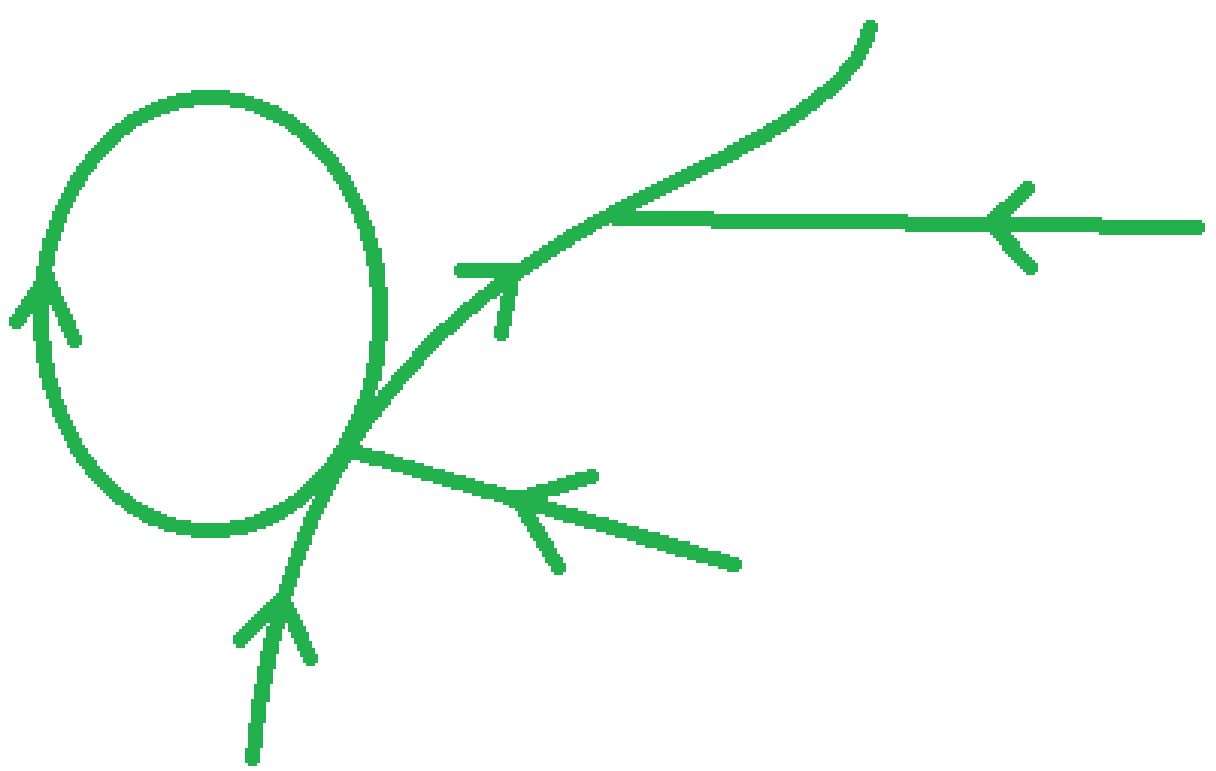

Figure 5.1: A general curve for the RHP.

transversing $\gamma$ according to its orientation, and the negative side on the right. If $\gamma_{0}:=\gamma \backslash$ intersection points and end points $\}$ and $Y$ be an analytic function in $\mathrm{C} \backslash \gamma$, the boundary values of $Y$, at positive and negative side of $\gamma$, in $x \in \gamma_{0}$ 
are denoted by

$$
\begin{aligned}
& Y_{+}(x)=\lim _{z \rightarrow x, z \in+\text { side }} Y(z) \\
& Y_{-}(x)=\lim _{z \rightarrow x, z \in-\text { side }} Y(z) .
\end{aligned}
$$

When $Y_{+}, Y_{-}$exist and are linked by a jump condition, $Y_{+}(x)=Y_{-}(x) j_{Y}(x)+$ $Z(x)$, hence $Y$ is the solution of a boundary value problem in the complex plane, the so-called Riemann Hilbert problem:

1. $Y$ is analytic in $\mathrm{C} \backslash \gamma$

2. $Y_{+}(x)=Y_{-}(x) j_{Y}(x)+Z(x), \quad x \in \gamma_{0}$,

where $j_{Y}$ is the continuos jump for $Y$ over $\gamma_{0}$. The solution for this problem is not unique, because by adding an entire function to $Y$ do not change the conditions of this problem. So to ensure uniqueness we impose an asymptotic condition,

3. $Y(z) \rightarrow Y(\infty), \quad z \rightarrow \infty$

This normalization condition also can be defined at other points, but is not usual. In the case of intersection points or end points, we need extra condition at those points.

Outline: The chapter is organized as follows. Section 5.2 contains basic notions of complex analysis. In section 5.3 we review the Cauchy type integrals. Section 5.4 gives the theory of scalar RHP. In section 5.5 we introduce the matrix RHP, study some cases and introduce the relation between orthogonal polynomials and matrix RHP. This is followed by the relation between both scalar, matrix RHP and singular integral equations.

\section{2}

\section{Complex Analysis}

A curve in the complex plane can be described via the parametrization

$$
z(t)=x(t)+i y(t), \quad a \leq t \leq b
$$

The curve is said to be continuous if $x(t)$ and $y(t)$ are continuous functions of $t$. Similarly, it is said to be differentiable if $x(t)$ and $y(t)$ are differentiable. A curve $\gamma$ is simple if it does not intersect itself, that is, $z\left(t_{1}\right) \neq z\left(t_{2}\right)$ if $t_{1} \neq t_{2}$ for $t_{1}, t_{2} \in[a, b]$, except that $z(b)=z(a)$ is allowed; in the latter case we say that $\gamma$ is a simple closed curve. A smooth $\operatorname{arc} C$ is one for which $z(t)$ is continuous. A contour is an arc consisting of a finite number of connected smooth arcs. 
A function $f(z)$ is said to be analytic at point $z_{0}$ of a region $R \subset \mathrm{C}$ if there exist $\epsilon>0$ and constants $a_{0}, a_{1}, \ldots$ such that for all points $z$ in the neighborhood $B\left(z_{0}, \epsilon\right) \subset R$ we have

$$
f(z)=a_{0}+a_{1}\left(z-z_{0}\right)+a_{2}\left(z-z_{0}\right)^{2}+\ldots
$$

Moreover, this power serie is unique and the coefficients are given by $a_{k}=$ $\frac{f^{(k)}\left(z_{0}\right)}{k !}$. A function is said to be entire when it is analytic at each point of the complex plane. An isolated singular point $z_{0}$ is a point where $f$ fails to be analytic. There are three types of singularities according to number of terms in the Laurent series

1. An isolated singularity at $z_{0}$ of $f(z)$ is said to be removable if $f(z)$ is bounded and $C_{0} \neq f\left(z_{0}\right)$ in $f(z)=\sum_{n=0}^{\infty} C_{n}\left(z-z_{0}\right)^{n}$. In this case by a slight redefinition of $f\left(z_{0}\right)$, the function $f(z)$ becomes analytic.

2. An isolated singularity at $z_{0}$ of $f(z)$ is said to be a pole if $f(z)=$ $\sum_{n=-N}^{\infty} C_{n}\left(z-z_{0}\right)^{n}$, where $N$ is the order of pole such that $\left(z-z_{0}\right)^{N} f(z)$ is analytic in a neighborhood of $z_{0}$

3. An isolated singular point that is neither removable nor a pole, is called an essential singular point.

A basic fact that has been used is Liouville's theorem.

Theorem (Liouville) If $f(z)$ is entire and bounded in the $z$ plane (including infinity), then $f(z)$ is a constant.

Cauchy's Theorem tells us that if $f(z)$ is analytic inside $C$, then $\oint_{C} f(z) d z=0$. Now we also recall the converse.

Theorem (Morera) If $f(z)$ is continuous in a domain $D$ and if

$$
\oint_{C} f(z) d z=0
$$

for every simple closed contour $C$ lying in $D$, then $f(z)$ is analytic in $D$.

\section{3}

\section{Cauchy Type integrals}

Many useful results for the RHP can be deduced from the Cauchy type integral. Given a smooth curve $\gamma$ (may be an arc or a closed contour) in the complex plane and a function $f: \gamma \rightarrow \mathrm{C}$, the Cauchy type integral is defined by

$$
C_{\gamma} f(z)=\frac{1}{2 \pi i} \int_{\gamma} \frac{f(x)}{x-z} d x .
$$


One observe that, in general, the Cauchy type integral maps functions on a contour to analytic functions off the contour. In this sense, we are going to describe a class of functions in which the Cauchy type integral has nice properties for the solution of the RHP.

Given $\gamma \subset \mathrm{C}$, a function satisfies the Hölder condition on $\gamma$ if for any two points $\tau$ and $\tau_{1}$ on $\gamma$, there exists $\Lambda>0$ such that

$$
\left|f(\tau)-f\left(\tau_{1}\right)\right| \leq \Lambda\left|\tau-\tau_{1}\right|^{\lambda}, \quad 0<\lambda \leq .1
$$

Note that If $\lambda=1$, the Hölder condition becomes the so-called Lipschitz condition and if $\lambda>1, \frac{d f}{d \tau}=0$ and hence $f$ must be constant.

Since the Cauchy type integral becomes ambiguous when $z$ be on $\gamma$, then to give it a unique meaning we must know how $z$ approaches $\gamma$. We denote the limiting values of $C_{\gamma} f$ in the same way that the boundary values of $Y$,

$$
\begin{aligned}
\lim _{z \rightarrow x, z \in+\text { side }} C_{\gamma} f(z) & =C_{\gamma}^{+} f(x) \\
\lim _{z \rightarrow x, z \in-\text { side }} C_{\gamma} f(z) & =C_{\gamma}^{-} f(x)
\end{aligned}
$$

Theorem (Sokhotski-Plemelj Formula) Let $\gamma$ be a smooth contour(closed or open) and let $f(\tau)$ satisfies a Hölder condition on $\gamma$. Then, for $x$ not an endpoint of $\gamma$, the limits of Cauchy type integral are given by

$$
\begin{aligned}
C_{\gamma}^{+} f(x) & =\frac{1}{2} f(x)+\frac{1}{2 \pi i} f_{\gamma} \frac{f(\tau)}{\tau-x} d \tau \\
C_{\gamma}^{-} f(x) & =-\frac{1}{2} f(x)+\frac{1}{2 \pi i} f_{\gamma} \frac{f(\tau)}{\tau-x} d \tau
\end{aligned}
$$

or in another words

$$
\begin{aligned}
C_{\gamma}^{+} f(x)-C_{\gamma}^{-} f(x) & =f(x) \\
C_{\gamma}^{+} f(x)-C_{\gamma}^{-} f(x) & =\frac{1}{\pi i} f_{\gamma} \frac{f(\tau)}{\tau-x} d \tau,
\end{aligned}
$$

where $f$ denotes the principal value integral defined by

$$
f_{\gamma} \frac{f(\tau) d \tau}{\tau-x}=\lim _{\epsilon \rightarrow 0} \int_{\gamma-\gamma_{\epsilon}} \frac{f(\tau) d \tau}{\tau-x}
$$

where $\gamma_{\epsilon}$ is the part of $\gamma$ that has length $2 \epsilon$ and is centered around $x$. The above framework is not sufficient to study RHPs for open contours, thereupon we need to know the behavior of $C_{\gamma} f(z)$ near the end points.

A function $f$ satisfies an Hölder condition on a open contour $\gamma$ if $f$ is Hölder 
away from the endpoints of $\gamma$ and if also at each endpoint $c, f$ satisfies

$$
f(x)=\frac{\bar{f}(x)}{(x-c)^{\alpha}}, \quad \alpha=m+i n, 0 \leq m<1,
$$

where $\bar{f}$ satisfies Hölder condition.

Let $\gamma$ be a bounded open contour from $a$ to $b$ and let $f$ satisfies a Hölder condition on $\gamma$. Consider $\gamma^{\prime} \subset \gamma$ be a curve with endpoints lying a finite distance from the endpoints $a$ and $b$ of $\gamma$. Then $C_{\gamma}^{ \pm} f$ satisfies the Hölder condition on $\gamma^{\prime}$. Among other things, the following holds for any endpoint $c=a, b$.

1. If $\alpha=0$, then

(a) As $z \rightarrow c, z \in \pm$ side,

$$
c_{\gamma} f(z)=\mp \frac{f(c)}{2 \pi i} \log \frac{1}{z-c}+g(z)
$$

(b) As $x \rightarrow c, x \in \gamma$

$$
c_{\gamma} f(x)=\mp \frac{f(c)}{2 \pi i} \log \frac{1}{x-c}+h(x) .
$$

Where $g$ and $h$ tend to definite limits at $c$. Also the upper sign is taken for $c=b$, and the lower for $c=a$. the branch cut for the logarithm is taken along $\gamma$.

2. Otherwise, If $\alpha \neq 0$,

(a) As $z \rightarrow c, z \in \pm$ side,

$$
c_{\gamma} f(z)=\mp \frac{e^{ \pm} i \alpha \pi}{2 i \sin (\alpha \pi)} \frac{\bar{f}(c)}{(z-c)^{\alpha}}+g(z)
$$

(b) As $x \rightarrow c, x \in \gamma$

$$
c_{\gamma} f(x)=\mp \frac{\cot (\alpha \pi)}{2 i} \frac{\bar{f}(c)}{(x-c)^{\alpha}}+h(x)
$$

such that if $m>0$ then for $0<\alpha^{*}<m$,

$$
|f(z)|<\frac{A}{|z-c|^{\alpha^{*}}}, \quad|g(x)|<\frac{B}{|x-c|^{\alpha^{*}}}
$$

For the proofs see [2]. 


\section{4}

\section{Scalar Riemann-Hilbert Problems}

The facts estudied above will now be used to solve scalar RHP. The index of a function $f(x)$ with respect to $\gamma$ is the increment of its argument in traversing a curve $\gamma$ in the positive direction, divided by $2 \pi$, that is

$$
\operatorname{indf}(x)=\frac{1}{2 \pi}[\arg f(x)]_{\gamma}=\frac{1}{2 \pi i}[\log f(x)]_{\gamma}=\frac{1}{2 \pi i} \int_{\gamma} d(\log f(x)) .
$$

Recall the solution of the simplest scalar RHP: Let $\gamma=\mathrm{R}$ and $j_{Y}$ be a Hölder continuous scalar function in R, then the following additive scalar RHP for $Y$ in $\mathrm{C}$ :

1. $Y$ is analytic in $\mathrm{C} \backslash \mathrm{R}$,

2. $Y_{+}(x)=Y_{-}(x)+j_{Y}(x), x \in \mathrm{R}$,

3. $Y(z) \rightarrow 0, z \rightarrow \infty$,

has the following unique solution as a Cauchy type integral

$$
Y(z)=C_{\mathrm{R}} j_{Y}(z)=\frac{1}{2 \pi i} \int_{\mathrm{R}} \frac{j_{Y}(x)}{x-z} d x .
$$

Indeed, the first condition is satisfied for the Cauchy type integral definition and Sokhotski-Plemelj Formulae gives for $x \in \mathrm{R}$

$$
C_{\mathrm{R}}^{+} j_{Y}(x)-C_{\mathrm{R}}^{-} j_{Y}(x)=j_{Y}(x),
$$

thence, $C_{\mathrm{R}} j_{Y}(x)$ satisfies the second condition. To show the third condition we use the geometric serie in the definition of $C_{\mathrm{R}} j_{Y}(x)$. Therefore

$$
\begin{aligned}
C_{\mathrm{R}} j_{Y}(z) & =\frac{1}{2 \pi i} \int_{\mathrm{R}} j_{Y}(x)\left[\left(-\frac{1}{z}\right) \frac{1}{1-\left(\frac{x}{z}\right)}\right] d x . \\
& =\frac{-1}{2 \pi z i} \int_{\mathrm{R}} j_{Y}(x)\left[\sum_{n=0}^{\infty}\left(\frac{x}{z}\right)^{n}\right] d x . \\
& =\sum_{n=0}^{\infty} \frac{1}{z^{n+1}}\left(\frac{-1}{2 \pi i} \int_{\mathrm{R}} j_{Y}(x) x^{n} d x\right) . \\
C_{\mathrm{R}} j_{Y}(z) & =\sum_{n=0}^{\infty} \frac{1}{z^{n+1}} a_{n},
\end{aligned}
$$

note that as $z \rightarrow \infty$ we have $C_{\mathrm{R}} j_{Y}(z) \rightarrow 0$. Therefore, there exists a solution, $C_{\mathrm{R}} j_{Y}(z)$, of the additive scalar RHP. Hereupon, to prove uniqueness, one can consider any other solution $h(z)$ of the additive scalar RHP. Thence the function $Z(z)=C_{\mathrm{R}} j_{Y}(z)-h(z)$ provides the following features:

1. $Z$ is an entire function, 
2. For $x \in \mathrm{R}$,

$$
\begin{aligned}
Z_{+}(x) & =C_{\mathrm{R}}^{+} j_{Y}(x)-h_{+}(z) \\
& =C_{\mathrm{R}}^{-} j_{Y}(x)+j_{Y}(z)-h_{-}(z)-j_{Y}(z) \\
Z_{+}(x) & =Z_{-}(x)
\end{aligned}
$$

3. $Z(z) \rightarrow 0, z \rightarrow \infty$.

By Liouville's theorem, $Z(z)=0$. Therefore, there is a unique solution $h(z)=C_{\mathrm{R}} j_{Y}(z)$.

The so-called multiplicative homogeneous RHP is formulated as follows:

1. $Y$ is analytic in $\mathrm{C} \backslash \mathrm{R}$,

2. $Y_{+}(x)=Y_{-}(x) j_{Y}(x), x \in \mathrm{R}$,

3. $Y(z) \rightarrow 1, z \rightarrow \infty$.

By applying the second condition to the logarithm

$$
(\log Y)_{+}(x)=\log \left(Y_{-}(x) j_{Y}\right)=(\log Y)_{-}(x)+\log j_{Y}(x) .
$$

Notice that this jump condition is similar to the jump condition of the additive RHP, but $\log j_{Y}(x)$ may not be well defined. In general, we suppose that ind $j_{Y}(x)=m$, and in this way $\log j_{Y}(x)$ may not be a Hölder continuous function. To overcome this issue we modify the jump function to $x^{-m} j_{Y}(x)$, such that

$$
\text { ind } \begin{aligned}
\left(x^{-m} j_{Y}(x)\right) & =\frac{1}{2 \pi i} \int_{\gamma} d\left(\log \left(x^{-m} j_{Y}(x)\right)\right) \\
& =\frac{1}{2 \pi i} \int_{\gamma} \frac{\left(x^{-m} j_{Y}(x)\right)^{\prime} d x}{\left(x^{-m} j_{Y}(x)\right)} \\
& =\frac{1}{2 \pi i} \int_{\gamma} \frac{\left((-m) x^{-m-1} j_{Y}(x)+x^{-m} j_{Y}^{\prime}(x)\right) d x}{\left(x^{-m} j_{Y}(x)\right)} \\
& =\frac{-m}{2 \pi i} \int_{\gamma} \frac{d x}{x}+\frac{1}{2 \pi i} \int_{\gamma} \frac{j_{Y}^{\prime}(x) d x}{j_{Y}(x)} \\
& =-m+\operatorname{ind} j_{Y}(x) \\
\text { ind }\left(x^{-m} j_{Y}(x)\right) & =0,
\end{aligned}
$$

hence $\log \left(x^{-m} j_{Y}(x)\right)$ is a Hölder continuous function. This recommends restating the second condition of multiplicative RHP in the following way

$$
Y_{+}(x)=\left(Y_{-}(x) x^{m}\right)\left(x^{-m} j_{Y}(x)\right)
$$

and by taking logarithm we have 


$$
(\log Y)_{+}(x)=\left(\log Y(x) x^{m}\right)_{-}+\log \left(x^{-m} j_{Y}(x)\right) .
$$

Therefore the solution of this multiplicative homogeneous RHP that satisfies the third condition is given by

$$
Y(z)=X(z) P_{m}(z)
$$

where $P_{m}(z)$ is an arbitrary polynomial of degree $m$ with leading coefficient 1 , and $X(z)$ is given by

$$
X(z)= \begin{cases}e^{\frac{1}{2 \pi i} \int_{\mathrm{R}} \frac{d x \log \left(x^{-m} j_{Y}(x)\right)}{x-z}} & , z \in \mathrm{C}^{+} \\ z^{-m} e^{\frac{1}{2 \pi i} \int_{\mathrm{R}} \frac{d x \log \left(x^{-m} j_{Y}(x)\right)}{x-z}} & , z \in \mathrm{C}^{-}\end{cases}
$$

This implies that there are three cases:

1. For $m=0$, we have at $z \in \mathrm{C} \backslash \mathrm{R}$

$$
Y(z)=X(z)=e^{\frac{1}{2 \pi i} \int_{\mathrm{R}} \frac{d x \log j_{Y}(x)}{x-z}} .
$$

As $\log j_{Y}(x)$ be Hölder continuous functions, this solution is unique, which is equivalent to

$$
\log Y(z)=\frac{1}{2 \pi i} \int_{\mathrm{R}} \frac{d x \log j_{Y}(x)}{x-z}
$$

notice that this corresponds to the Cauchy type solution of the equivalent additive $\operatorname{RHP}(5.16)$.

2. In the case of $m>0$, there exist $m$ linearly independent solutions at $z \in \mathrm{C} \backslash \mathrm{R}$

$$
Y_{m}(z)=X(z) P_{m}(z),
$$

because the asymptotic condition does not restrict $P_{m}$,

$$
Y(z) \rightarrow z^{-m} P_{m}, \quad z \rightarrow \infty
$$

notice that for this limit vanishing at infinity, we require $P_{m}(z)=$ $a_{0}+a_{1} z+a_{2} z^{2}+\ldots+a_{m-1} z^{m-1}$ such that it has $m$ arbitrary constants, because

$$
z^{m} P_{m} \cong O\left(z^{-1}\right), \quad z \rightarrow \infty
$$

3. Considering $m<0$ in the solution (5.23). Then

$$
Y(z)=X(z) P_{m}(z)=0
$$

is the only solution that satisfies

$$
Y(z) \rightarrow 0, \quad z \rightarrow \infty
$$


because one must take $P_{m}(z)=0$ to remove the growth at infinity of $X(z)$.

Now we are going to look at inhomogeneous scalar RHP: Let $j_{Y}(x), k_{Y}(x)$ be Hölder continuous functions in R, then

1. $Y$ is analytic in $\mathrm{C} \backslash \mathrm{R}$,

2. $Y_{+}(x)=Y_{-}(x) j_{Y}(x)+k_{Y}(x), x \in \mathrm{R}$,

3. $Y(z) \rightarrow 0, z \rightarrow \infty$.

The solution of this problem is derived from the solution of the homogeneous RHP. Actually, if ind $j_{Y}(x)=n$, dividing the jump condition by the homogeneous boundary solution $X_{+}(z)$ for $P_{m}=1$, and taking into account that $X_{+}(x)=X_{-}(x) j_{Y}(x)$, we have

$$
\begin{aligned}
\frac{Y_{+}(x)}{X_{+}(x)} & =\frac{Y_{-}(x) j_{Y}(x)}{X_{+}(x)}+\frac{k_{Y}(x)}{X_{+}(x)} \\
& =\frac{Y_{-}(x) j_{Y}(x)}{X_{-}(x) j_{Y}(x)}+\frac{k_{Y}(x)}{X_{+}(x)} \\
\frac{Y_{+}(x)}{X_{+}(x)} & =\frac{Y_{-}(x)}{X_{-}(x)}+\frac{k_{Y}(x)}{X_{+}(x)}
\end{aligned}
$$

hence we obtain a jump condition for $\frac{Y(z)}{X(z)}$, and by the Cauchy type integral

$$
\frac{Y(z)}{X(z)}=\frac{1}{2 \pi i} \int_{\mathrm{R}} \frac{d x k_{Y}(x)}{X_{+}(x)(x-z)}+P_{n}(z)
$$

next to find the solution at infinity,

$$
\frac{Y(z)}{X(z)}=O\left(z^{-1}\right)
$$

Anew, there are three solutions that accomplish this requirement in the following way:

1. if $n=0$, then $P_{n}(z)=0$ and hence

$$
Y(z)=\frac{X(z)}{2 \pi i} \int_{\mathrm{R}} \frac{d x k_{Y}(x)}{X_{+}(x)(x-z)}
$$

is a unique solution of the inhomogeneous scalar RHP.

2. if $n>0$, then there exist $n$ linearly independent solutions due to unconstrained $n$ arbitrary constants of $P_{n}(z)$ by the asymptotic condition. 
3. If $n<0$, then $P_{n}(z)=0$ and the asymptotic expansion is given by

$$
\begin{aligned}
Y(z) & =\frac{X(z)}{2 \pi i} \int_{\mathrm{R}} \frac{d x k_{Y}(x)}{X_{+}(x)(x-z)} \\
& =\frac{X(z)}{2 \pi i}\left(\frac{1}{-z} \int_{\mathrm{R}} \frac{d x k_{Y}(x)}{X_{+}(x)} \frac{1}{\left(1-\frac{x}{z}\right)}\right) \\
& =\frac{-X(z)}{2 \pi i} \int_{\mathrm{R}} \frac{d x k_{Y}(x)}{X_{+}(x) z}\left(\sum_{i=0}^{\infty} \frac{x^{i}}{z^{i}}\right) \\
& =\frac{-X(z)}{2 \pi i} \int_{\mathrm{R}} \frac{d x k_{Y}(x)}{X_{+}(x)}\left(\sum_{i=0}^{|n|-1} \frac{x^{i}}{z^{i+1}}+\sum_{i=|n|}^{\infty} \frac{x^{i}}{z^{i+1}}\right) \\
Y(z) & =\frac{-1}{2 \pi i} \int_{\mathrm{R}} \frac{d x k_{Y}(x)}{X_{+}(x)}\left(\sum_{i=0}^{|n|-1} \frac{x^{i} X(z)}{z^{i+1}}+\sum_{i=|n|}^{\infty} \frac{x^{i} X(z)}{z^{i+1}}\right) .
\end{aligned}
$$

Since $X(z) \cong z^{|n|}$ at $z \rightarrow \infty$, thus when $z \rightarrow \infty$ notice that $Y(z)$ vanishes at infinity when the first sum vanishes

$$
Y(z) \cong \frac{-1}{2 \pi i} \int_{\mathrm{R}} \frac{d x k_{Y}(x)}{X_{+}(x)}\left(\sum_{i=0}^{|n|-1} \frac{x^{i} z^{|n|}}{z^{i+1}}+\sum_{i=|n|}^{\infty} \frac{x^{i} z^{|n|}}{z^{i+1}}\right),
$$

This happens if and only if for all $i=0,1, \ldots,|n|-1$

$$
\int_{\mathrm{R}} \frac{d x k_{Y}(x) x^{i}}{X_{+}(x)}=0 .
$$

\section{5}

\section{Matrix Riemann-Hilbert Problems}

Given an oriented curve $\gamma$ in the complex plane C. Let $j_{Y}: \gamma \rightarrow \mathrm{C}^{k \times k}$ be an invertible $k \times k$ matrix function such that all its matrix elements satisfy a Hölder condition. We can say that an $j \times k$ matrix function $Y: \mathrm{C} \backslash \gamma \rightarrow \mathrm{C}^{j \times k}$ is a solution of $\operatorname{RHP}\left(\gamma, j_{Y}\right)$ if

1. $Y_{i l}(z)$ are analytic in $\mathrm{C} \backslash \gamma$.

2. $Y_{+}(x)=Y_{-}(x) j_{Y}(x), \quad x \in \gamma$

If, in addition, $j=k$ and

3. $Y(z) \cong I_{k}, \quad z \rightarrow \infty$,

where $I_{k}$ denotes the $k \times k$ identity matrix. We say that $Y(z)$ solves the matrix RHP and can be reduced to the analysis of an array of scalar RHPs. If one could not reduce the matrix RHP to an array of scalar RHPs we can study this problem by singular integral equations. This will be described in the next 
section.

The shape of $j_{Y}$ determines the type of matrix RHP. Thus, let us first

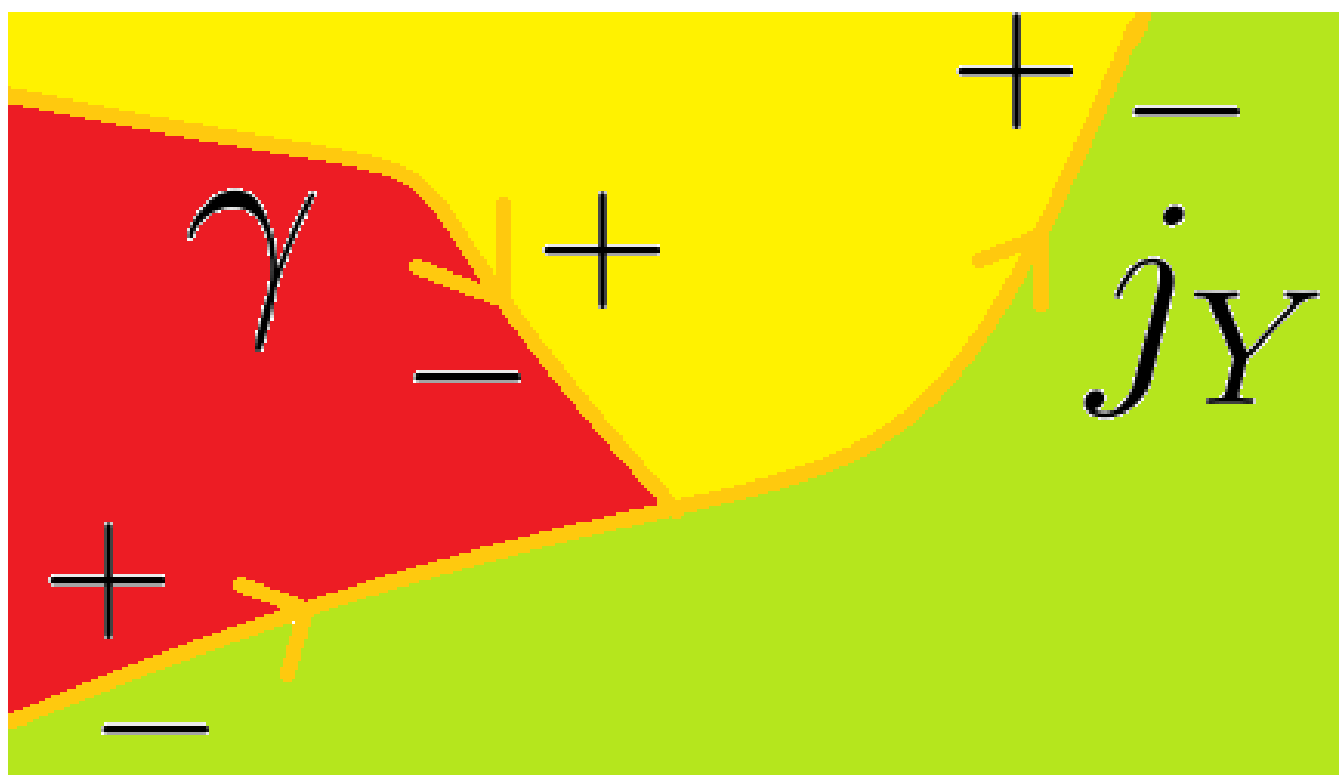

Figure 5.2: An arbitrary oriented curve $\gamma$ for a given jump condition $j_{Y}$.

discuss some cases: Diagonal matrix RHPs, Constant matrix RHPs, Rational matrix RHPs, Triangular matrix RHPs and characterization of orthogonal polynomials.

\subsection{1}

\section{Diagonal Matrix Riemann Hilbert problems}

In this case $j_{Y}$ is a diagonal matrix such that $\left|j_{Y}\right| \neq 0$. Thus $Y$ solves the following RHP:

1. $Y_{i j}$ are analytic in $\mathrm{C} \backslash \gamma$.

2. For any $x \in \gamma$,

$$
Y_{+}(x)=Y_{-}(x)\left(\begin{array}{cccc}
j_{Y, 11}(x) & 0 & \cdots & 0 \\
0 & j_{Y, 22}(x) & \cdots & 0 \\
\vdots & \vdots & \ddots & \vdots \\
0 & 0 & \cdots & j_{Y, k k}(x)
\end{array}\right)
$$

3. As $z \rightarrow \infty$,

$$
Y(z) \cong I_{k}
$$

Letting ind $j_{Y, i i}(x)=0$, this problem can be separated into $k$ scalar RHP, in the following way:

1. $Y_{i i}$ are analytic in $\mathrm{C} \backslash \gamma$. 
2. For any $x \in \gamma$,

$$
Y_{+, i i}(x)=Y_{-, i i}(x) j_{Y, i i}
$$

3. As $z \rightarrow \infty$,

$$
Y_{i i}(z) \cong 1
$$

where $i=1,2, \ldots, k$. Then each of these problems has a unique solution given by Sokhotski-Plemelj formula

$$
Y_{i i}(z)=e^{\frac{1}{2 \pi i} \int_{\gamma} \frac{d x \log j_{Y, i i}}{x-z}}
$$

which is a solution of the multiplicative scalar RHP. Therefore a unique solution of the diagonal matrix RHP is given by

$$
Y(z)=\left(\begin{array}{cccc}
e^{\frac{1}{2 \pi i} \int_{\gamma} \frac{d x \log j_{Y, 11}}{x-z}} & 0 & \cdots & 0 \\
0 & e^{\frac{1}{2 \pi i} \int_{\gamma} \frac{d x \log j_{Y, 22}}{x-z}} & \cdots & 0 \\
\vdots & \vdots & \ddots & \vdots \\
0 & 0 & \cdots & e^{\frac{1}{2 \pi i} \int_{\gamma} \frac{d x \log j_{Y, k k}}{x-z}}
\end{array}\right)
$$

\section{5 .2}

\section{Constant Matrix Riemann Hilbert problems}

In this case $j_{Y}$ is a constant matrix with $\left|j_{Y}\right| \neq 0$, also is diagonalizable, $j_{Y}=U D_{Y} U^{-1}$. Accordingly $Y$ solves the following RHP:

1. $Y_{i j}$ are analytic in $\mathrm{C} \backslash \gamma$.

2. For any $x \in \gamma$,

$$
\begin{aligned}
Y_{+}(x) & =Y_{-}(x)\left(\begin{array}{cccc}
j_{Y, 11} & j_{Y, 12} & \cdots & j_{Y, 1 k} \\
j_{Y, 21} & j_{Y, 22} & \cdots & j_{Y, 2 k} \\
\vdots & \vdots & \ddots & \vdots \\
j_{Y, k 1} & j_{Y, k 2} & \cdots & j_{Y, k k}
\end{array}\right) \\
& =Y_{-}(x) U\left(\begin{array}{cccc}
D_{Y, 11} & 0 & \cdots & 0 \\
0 & D_{Y, 22} & \cdots & 0 \\
\vdots & \vdots & \ddots & \vdots \\
0 & 0 & \cdots & D_{Y, k k}
\end{array}\right) U^{-1}
\end{aligned}
$$

3. As $z \rightarrow \infty$,

$$
Y(z) \cong I_{k}
$$

By multiplying $U$ from the left by the jump condition, we obtain 


$$
Y_{+}(x) U=Y_{-}(x) U\left(\begin{array}{cccc}
D_{Y, 11} & 0 & \cdots & 0 \\
0 & D_{Y, 22} & \cdots & 0 \\
\vdots & \vdots & \ddots & \vdots \\
0 & 0 & \cdots & D_{Y, k k}
\end{array}\right)
$$

which corresponds to a diagonal matrix RHP with the following solution:

$$
Y(z) U=\left(\begin{array}{cccc}
e^{\frac{1}{2 \pi i} \int_{\gamma} \frac{d x \log D_{Y, 11}}{x-z}} & 0 & \cdots & 0 \\
0 & e^{\frac{1}{2 \pi i} \int_{\gamma} \frac{d x \log D_{Y, 22}}{x-z}} & \cdots & 0 \\
\vdots & \vdots & \ddots & \vdots \\
0 & 0 & \cdots & e^{\frac{1}{2 \pi i} \int_{\gamma} \frac{d x \log D_{Y, k k}}{x-z}}
\end{array}\right)
$$

therefore a unique solution of the constant matrix RHP is given by

$$
Y(z)=\left(\begin{array}{cccc}
e^{\frac{1}{2 \pi i} \int_{\gamma} \frac{d x \log D_{Y, 11}}{x-z}} & 0 & \cdots & 0 \\
0 & e^{\frac{1}{2 \pi i} \int_{\gamma} \frac{d x \log D_{Y, 22}}{x-z}} & \cdots & 0 \\
\vdots & \vdots & \ddots & \vdots \\
0 & 0 & \cdots & e^{\frac{1}{2 \pi i} \int_{\gamma} \frac{d x \log D_{Y, k k}}{x-z}}
\end{array}\right) U^{-1}
$$

\subsection{3}

\section{Rational Matrix Riemann Hilbert Problems}

Now the matrix elements of $j_{Y}$ are rational functions, $j_{Y, i j}(z)=\frac{p_{i j}(z)}{q_{i j}(z)}$ such that $p_{i j}(z), q_{i j}(z)$ are polynomials. Hence $j_{Y}$ can be rewritten as $j_{Y}(z)=\frac{P(z)}{r(z)}$, where $P(z)$ is a matrix whose elements are polynomials, and $r(z)$ is a polynomial. Thereupon $Y$ solves the following RHP:

1. $Y_{i j}$ are analytic in $\mathrm{C} \backslash \gamma$.

2. For any $x \in \gamma$,

$$
Y_{+}(x)=Y_{-}(x) \frac{1}{r(x)}\left(\begin{array}{cccc}
P_{11}(x) & P_{12}(x) & \cdots & P_{1 k}(x) \\
P_{21}(x) & P_{22}(x) & \cdots & P_{2 k}(x) \\
\vdots & \vdots & \ddots & \vdots \\
P_{k 1}(x) & P_{k 2}(x) & \cdots & P_{k k}(x)
\end{array}\right)
$$

3. As $z \rightarrow \infty$,

$$
Y(z) \cong I_{k}
$$

Notice that $P(z)$ can be factorized as

$$
P(z)=P_{+}(z) D(z) P_{-}(z),
$$


where $D(z)$ is a diagonal polynomial matrix, such that $\left|P_{+}\right|$and $\left|P_{-}\right|$are polynomials that do not have zeros in the + side and - side respectively. Then, by decomposing $r$ into factors, $r(x)=r_{+}(x) r_{-}(x)$, where $r_{+}(x)$ and $r_{-}(x)$ are polynomials that do not have zeros in the + side and - side respectively, we have the following diagonal matrix RHP:

1. $Y_{i j}$ are analytic in $\mathrm{C} \backslash \gamma$.

2. For any $x \in \gamma$,

$$
r_{+}(x) P_{+}^{-1}(x) Y_{+}(x)=Y_{-}(x) \frac{P_{-}(x)}{r_{-}(x)}\left(\begin{array}{cccc}
D_{1}(x) & 0 & \cdots & 0 \\
0 & D_{2}(x) & \cdots & 0 \\
\vdots & \vdots & \ddots & \vdots \\
0 & 0 & \cdots & D_{k}(x)
\end{array}\right)
$$

3. As $z \rightarrow \infty$,

$$
Y(z) \cong I_{k}
$$

In general, $D_{i}(x)=D_{i+}(x) D_{i-}(x)$, where $D_{i+}(x)$ and $D_{i-}(x)$ are polynomials that do not have zeros in the + side and - side respectively, we obtain

$$
\begin{gathered}
\left(\begin{array}{ccc}
\frac{1}{D_{1-}(x)} & \cdots & 0 \\
\vdots & \ddots & \vdots \\
0 & \cdots & \frac{1}{D_{k-}(x)}
\end{array}\right) r_{+}(x) P_{+}^{-1}(x) Y_{+}(x)= \\
\quad=Y_{-}(x) \frac{P_{-}(x)}{r_{-}(x)}\left(\begin{array}{ccc}
D_{1+}(x) & \cdots & 0 \\
\vdots & \ddots & \vdots \\
0 & \cdots & D_{k+}(x)
\end{array}\right)
\end{gathered}
$$

Hence, by the analyticity of polynomials in each side, we have

$$
\begin{aligned}
\left(\begin{array}{ccc}
\frac{1}{D_{1-}(x)} & \cdots & 0 \\
\vdots & \ddots & \vdots \\
0 & \cdots & \frac{1}{D_{k-}(x)}
\end{array}\right) & =Y_{-}(x) \frac{P_{-}(x)}{r_{-}(x)} \\
r_{+}(x) P_{+}^{-1}(x) Y_{+}(x) & =\left(\begin{array}{ccc}
D_{1+}(x) & \cdots & 0 \\
\vdots & \ddots & \vdots \\
0 & \cdots & D_{k+}(x)
\end{array}\right) .
\end{aligned}
$$


Therefore, in general $Y$ is determined by the following boundary values

$$
\begin{gathered}
Y_{-}(x)=r_{-}(x) P_{-}(x)^{-1}\left(\begin{array}{ccc}
\frac{1}{D_{1-}(x)} & \cdots & 0 \\
\vdots & \ddots & \vdots \\
0 & \cdots & \frac{1}{D_{k-}(x)}
\end{array}\right) \\
Y_{+}(x)=\frac{P_{+}(x)}{r_{+}(x)}\left(\begin{array}{ccc}
D_{1+}(x) & \cdots & 0 \\
\vdots & \ddots & \vdots \\
0 & \cdots & D_{k+}(x)
\end{array}\right)
\end{gathered}
$$

\subsection{4}

\section{Triangular Matrix Riemann Hilbert Problems}

Here $j_{Y}$ is either an upper or lower triangular matrix with $\left|j_{Y}\right| \neq 0$. Thence $Y$ solves the following RHP:

1. $Y_{i j}$ are analytic in $\mathrm{C} \backslash \gamma$.

2. For any $x \in \gamma$,

$$
Y_{+}(x)=Y_{-}(x)\left(\begin{array}{cccc}
j_{Y, 11}(x) & j_{Y, 12}(x) & \cdots & j_{Y, 1 k}(x) \\
0 & j_{Y, 22}(x) & \cdots & j_{Y, 2 k}(x) \\
\vdots & \vdots & \ddots & \vdots \\
0 & 0 & \cdots & j_{Y, k k}(x)
\end{array}\right)
$$

3. As $z \rightarrow \infty$,

$$
Y(z) \cong I_{k}
$$

First of all, to have unique solution we assume ind $j_{Y, i i}(x)=0$ for $i=1,2, \ldots, k$. While later, by multiplying and comparing the second and third condition of the triangular RHP respectively, we decompose step by step the first row of $Y$ into an array of scalar RHPs:

The homogeneous scalar RHP,

1. $Y_{11}$ is analytic in $\mathrm{C} \backslash \gamma$,

2. $Y_{+, 11}(x)=Y_{-, 11}(x) j_{Y, 11}(x), \quad x \in \gamma$,

3. $Y_{11}(z) \rightarrow 1, \quad z \rightarrow \infty$,

yields $Y_{11}$, then the inhomogeneous scalar RHP,

1. $Y_{12}$ is analytic in $\mathrm{C} \backslash \gamma$,

2. $Y_{+, 12}(x)=Y_{-, 12}(x) j_{Y, 22}(x)+Y_{-, 11}(x) j_{Y, 12}(x), \quad x \in \mathrm{R}$, 
3. $Y_{12}(z) \rightarrow 0, \quad z \rightarrow \infty$,

yields $Y_{12}$, hence consecutively the inhomogeneous scalar RHP,

1. $Y_{1 k}$ is analytic in $\mathrm{C} \backslash \gamma$,

2. $Y_{+, 1 k}(x)=Y_{-, 1 k}(x) j_{Y, k k}(x)+\sum_{n=1}^{k-1} Y_{-, 1 n}(x) j_{Y, n k}(x), \quad x \in \mathrm{R}$,

3. $Y_{1 k}(z) \rightarrow 0, \quad z \rightarrow \infty$,

yields $Y_{1 k}$. Notice that the first row was decomposed independently of the other rows. Hence we can reduce each row $m$ independent of the other rows to an array of scalar RHPs:

1. $Y_{m 1}, \ldots, Y_{m k}$ is analytic in $\mathrm{C} \backslash \gamma$,

2. For any $x \in \mathrm{R}$

$$
\begin{aligned}
Y_{+, m 1}(x)= & Y_{-, m 1}(x) j_{Y, 11}(x) \\
& \ddots \\
Y_{+, m k}(x)= & Y_{-, m k}(x) j_{Y, k k}(x)+\sum_{n=1}^{k-1} Y_{-, m n}(x) j_{Y, n k}(x)
\end{aligned}
$$

3. As $z \rightarrow \infty$,

$$
Y_{m n}(z) \rightarrow \delta_{m n}
$$

\section{6}

\section{Characterization of Orthogonal Polynomials}

Let us now consider a matrix RHP: $Y_{n}$ and $j_{Y}$ being matrix functions, with $j_{Y}(x)=\left(\begin{array}{cc}1 & w(x) \\ 0 & 1\end{array}\right)$, where $w(x)$ defines a finite measure over R. This allows to establish a scalar product and by the Gram-Schimdt ortogonalization one determines corresponding orthogonal polynomials $p_{n}(x)$. Those can be characterized through the following matrix RHP for $Y_{n}$

1. $Y_{n, 11}(z), Y_{n, 12}(z), Y_{n, 21}(z), Y_{n, 22}(z)$, are analytic in $\mathrm{C}^{+}, \mathrm{C}^{-}$,

2. $\quad Y_{n+}(x)=Y_{n-}(x) j_{Y}(x), \quad x \in \mathrm{R}$,

3. $\quad Y(z) \cong\left(\begin{array}{cc}z^{n}+O\left(z^{n-1}\right) & O\left(z^{-n-1}\right) \\ O\left(z^{n-1}\right) & z^{-n}+O\left(z^{-n-1}\right)\end{array}\right), z \rightarrow \infty$,

where, by Liouville theorem, $Y_{n, 11}=p_{n}$ and we have a scalar RHP for $Y_{n, 12}$, with solution $Y_{n, 12}(z)=\frac{1}{2 \pi i} \int_{\mathrm{R}} \frac{p_{n}(x) w(x)}{x-z} d x$. Analogously for the rest. Therefore the unique solution of this matrix RHP is given by 


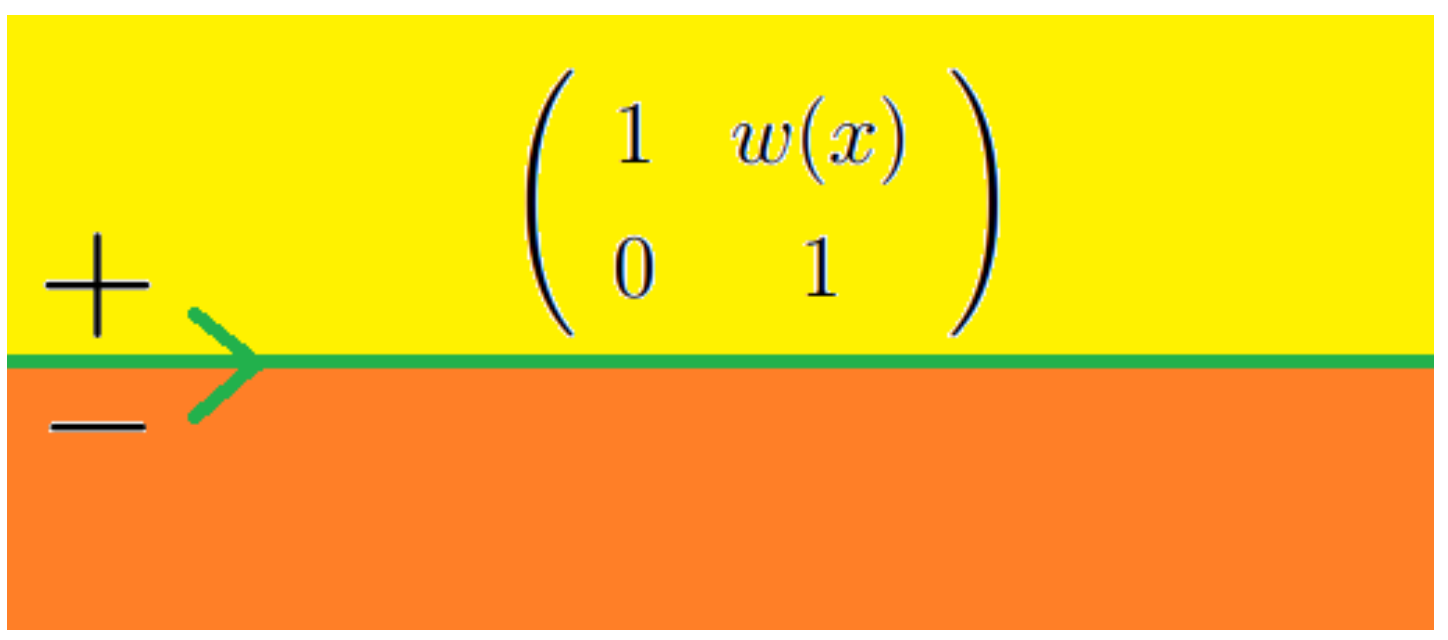

Figure 5.3: Representation of the orthogonal polynomial.

$$
Y_{n}(z)=\left(\begin{array}{cc}
p_{n}(z) & \frac{1}{2 \pi i} \int_{\mathrm{R}} \frac{p_{n}(x) w(x) d x}{x-z} \\
-\frac{2 \pi i}{h_{n-1}} p_{n-1}(z) & -\frac{1}{h_{n-1}} \int_{\mathrm{R}} \frac{p_{n-1}(x) w(x) d x}{x-z}
\end{array}\right),
$$

hence the correlation kernel is expressed in terms of $Y_{N \pm}(x)$ as follows

$$
K_{N}\left(\lambda_{i}, \lambda_{j}\right)=\frac{e^{-\frac{1}{2} N\left(V\left(\lambda_{i}\right)+V\left(\lambda_{j}\right)\right)}}{2 \pi i\left(\lambda_{i}-\lambda_{j}\right)}\left(\begin{array}{ll}
0 & 1
\end{array}\right) Y_{N+}^{-1}\left(\lambda_{j}\right) Y_{N-}\left(\lambda_{i}\right)\left(\begin{array}{l}
1 \\
0
\end{array}\right)
$$

\section{7}

\section{Singular Integral Equations}

In some cases, matrix RHP can not be reduced to an array of scalar RHPs. But these cases can be solved by singular integral equations, because matrix RHP can be represented as singular integral equations.

In this section we introduce the singular integral equations and its relation to both scalar and matrix RHPs. First of all, consider the so-called linear integral equation for $y(x)$ given by

$$
g(x) y(x)=f(x)+\int_{\gamma} K\left(x, x^{\prime}\right) y\left(x^{\prime}\right) d x^{\prime},
$$

where $g(x), f(x)$ and $K\left(x, x^{\prime}\right)$ are integrable functions. Moreover, the kernel $K\left(x, x^{\prime}\right)$ can be a distribution. If $g(x)=0$ on $\gamma$, the integral equation is of the first kind, and in the other case is of the second kind.

An integral equation is usually called singular if

1. the contour increases to infinity,

2. the kernel is not bounded.

Hence a singular kernel can have the following form 


$$
K\left(x, x^{\prime}\right)=\frac{-h(x)}{\pi i\left(x^{\prime}-x\right)}
$$

in this case, the contour integral is given by the principal value integral. Thus we have the following singular integral equation

$$
g(x) y(x)-f_{\gamma}\left(\frac{-h(x)}{\pi i\left(x^{\prime}-x\right)}\right) y\left(x^{\prime}\right) d x^{\prime}=f(x)
$$

or

$$
g(x) y(x)+\frac{h(x)}{\pi i} f_{\gamma} \frac{y\left(x^{\prime}\right) d x^{\prime}}{x^{\prime}-x}=f(x),
$$

where $f(x), g(x), h(x)$ satisfy the Hölder condition on $\gamma$. If we consider

$$
Y(z)=\frac{1}{2 \pi i} \int_{\gamma} \frac{y(x) d x}{x-z}
$$

which is a Cauchy type integral. Then by Sokhotski-Plemelj formula we obtain

$$
\begin{aligned}
Y_{+}(x)-Y_{-}(x) & =y(x) \\
Y_{+}(x)+Y_{-}(x) & =\frac{1}{\pi i} \int_{\gamma} \frac{y\left(x^{\prime}\right) d x^{\prime}}{x^{\prime}-x}
\end{aligned}
$$

replacing these relations in the singular integral equation (5.47), we find

$$
g(x)\left(Y_{+}(x)-Y_{-}(x)\right)+h(x)\left(Y_{+}(x)+Y_{-}(x)\right)=f(x)
$$

reducing to the following way

$$
Y_{+}(x)=\left(\frac{g(x)-h(x)}{g(x)+h(x)}\right) Y_{-}(x)+\left(\frac{f(x)}{g(x)+h(x)}\right)
$$

which corresponds to a inhomogeneous scalar RHP, such that

$$
\begin{aligned}
j_{Y}(x) & =\frac{g(x)-h(x)}{g(x)+h(x)}, \\
k_{Y}(x) & =\frac{f(x)}{g(x)+h(x)} .
\end{aligned}
$$

This equivalence between scalar RHPs and singular integral equations can be extended to the matrix RHPs. For this purpose we recall the jump condition of the matrix RHP,

$$
Y_{+}(x)=Y_{-}(x) j_{Y}(x) .
$$

Since, $\left|i_{Y}\right| \neq 0$ and $j_{Y}(x)-I$ satisfy $\left|j_{Y}(x)-I\right|<C$ for a $C>0$, defining $g \equiv j_{Y}-I$, this jump condition can be written as

$$
Y_{+}(x)=Y_{-}(x) g(x)+Y_{-}(x),
$$


subtracting $I$ from both terms,

$$
Y_{+}(x)-I=Y_{-}(x) g(x)+Y_{-}(x)-I
$$

and ordering appropriately

$$
\left(Y_{+}(x)-I\right)-\left(Y_{-}(x)-I\right)=Y_{-}(x) g(x),
$$

by the Sokhotski-Plemelj formula we obtain

$$
\left(Y_{+}(x)-I\right)+\left(Y_{-}(x)-I\right)=\frac{1}{\pi i} \int_{\gamma} \frac{Y_{-}\left(x^{\prime}\right) g\left(x^{\prime}\right)}{x^{\prime}-x} d x^{\prime},
$$

using the jump condition we get

$$
Y_{-}(x) j_{Y}(x)+Y_{-}(x)-2 I=\frac{1}{\pi i} \int_{\gamma} \frac{Y_{-}\left(x^{\prime}\right) g\left(x^{\prime}\right)}{x^{\prime}-x} d x^{\prime},
$$

adding and subtracting $Y_{-}(x)$ we have

$$
Y_{-}(x)\left(j_{Y}(x)-I\right)+2 Y_{-}(x)-2 I=\frac{1}{\pi i} \int_{\gamma} \frac{Y_{-}\left(x^{\prime}\right) g\left(x^{\prime}\right)}{x^{\prime}-x} d x^{\prime}
$$

by the definition of $g$ we obtain

$$
Y_{-}(x)-I=-\frac{Y_{-}(x) g(x)}{2}+\frac{1}{2 \pi i} \int_{\gamma} \frac{Y_{-}\left(x^{\prime}\right) g\left(x^{\prime}\right)}{x^{\prime}-x} d x^{\prime}
$$

deforming the contour $\gamma$ to two contours, $\gamma-\gamma_{\epsilon}$ and $C_{\epsilon}$, where $C_{\epsilon}$ is a semicircle of radius $\epsilon$ centered at $x$, we have

$$
Y_{-}(x)-I=\lim _{\epsilon \rightarrow 0} \frac{1}{2 \pi i} \int_{C_{\epsilon}} \frac{Y_{-}\left(x^{\prime}\right) g\left(x^{\prime}\right)}{x^{\prime}-x} d x^{\prime}+\frac{1}{2 \pi i} \int_{\gamma} \frac{Y_{-}\left(x^{\prime}\right) g\left(x^{\prime}\right)}{x^{\prime}-x} d x^{\prime},
$$

therefore by the definition of the principal value integral, we obtain

$$
Y_{-}(x)-I=\frac{1}{2 \pi i} \int_{\gamma} \frac{Y_{-}\left(x^{\prime}\right) g\left(x^{\prime}\right)}{x^{\prime}-(x-i \epsilon)} d x^{\prime}
$$

which is equivalent to

$$
Y_{-}(x)-I=\int_{\gamma} K\left(x, x^{\prime}\right) Y_{-}\left(x^{\prime}\right) d x^{\prime}
$$

such that $K\left(x, x^{\prime}\right)=\frac{1}{2 \pi i} \frac{g\left(x^{\prime}\right)}{x^{\prime}-(x-i \epsilon)}$. As shown above this singular integral equation of the second kind is equivalent to the matrix RHP. 


\section{6 \\ Large asymptotics of orthogonal polynomials: The Riemann- Hilbert approach}

Many statistical quantities of Hermitian Random Matrices can be reduced to calculations involving large asymptotics of orthogonal polynomials. In this chapter we present the Riemann-Hilbert approach to the large asymptotics of orthogonal polynomials such that we follow the exposure of [17]. This approach is based on the steepest descent method, which provides an asymptotic solution to the RHP, as $N \rightarrow \infty$. For more detail the reader should consult $[13,19,20]$.

\section{1}

\section{Introduction}

In the 1990s, the steepest descendent method was developed by Percy Deift and Xin Zhou[?] and then applied to orthogonal polynomials by Deift, Kriecherbauer, McLaughlin, Venakides and Zhou[19, 20]. Its aplication span from universality, double scaling limit[17], Fredhom kernel, to painleve equations[18], amongs others. In general terms, the steepest descent method consists in performing a number of explicit and invertible transformations $Y_{N} \rightarrow T_{N} \rightarrow \ldots \rightarrow R_{N}$, provided that for the last equivalent RHP both the jump matrix and the behavior at infinity are closed to the identity. After tracing back the transformations, $R_{N} \rightarrow \ldots \rightarrow T_{N} \rightarrow Y_{N}$, we obtain the asymptotic solution for RHP that characterized the orthogonal polynomials.

Outline: The chapter is organized as follows. In section 6.2 we normalize the asymptotic condition of the RHP, supported from the Heine formula for orthogonal polynomials. In section 6.3 we recuperate the behavior at infinity of the jump matrix of the RHP except in the edge points. In section 6.4 we regularize the RHP in the edge points. Section 6.5 gives the large asymptotics of orthogonal polynomials. This is followed with concluding remarks in Section 6.6 .

\section{2}

\section{First transformation of the RHP}

In this chapter we transform the asymptotic condition in the RHP of $Y_{N}$, that is non constant, for a condition asymptotically closed to the identity matrix. 
Chapter 6. Large asymptotics of orthogonal polynomials: The Riemann-Hilbert approach

Namely, $T_{N}(z)=I+O\left(z^{-1}\right)$, as $z \rightarrow \infty$. For this reason, we define the following transformation in the RHP:

$$
T_{N}(z)=e^{-\frac{N l}{2} \sigma_{3}} Y_{N}(z) e^{-N\left[g(z)-\frac{l}{2}\right] \sigma_{3}}, \quad \sigma_{3}=\left(\begin{array}{cc}
1 & 0 \\
0 & -1
\end{array}\right),
$$

where $g$ is an analytic function in $\mathrm{C} \backslash \mathrm{R}, l$ is a constant, $\sigma_{3}$ is a Pauli matrix and for every function $f$ we have

$$
\begin{aligned}
e^{f(z) \sigma_{3}}=\sum_{i} \frac{f^{i}(z) \sigma_{3}^{i}}{i !} & =\sum_{i} \frac{f^{2 i+1}(z) \sigma_{3}^{2 i+1}}{(2 i+1) !}+\sum_{i} \frac{f^{2 i}(z) \sigma_{3}^{2 i}}{(2 i) !} \\
& =\sum_{i} \frac{f^{2 i+1}(z) \sigma_{3}}{(2 i+1) !}+\sum_{i} \frac{f^{2 i}(z) I}{(2 i) !} \\
& =\sum_{i} \frac{f^{2 i+1}(z)}{(2 i+1) !}\left(\begin{array}{cc}
1 & 0 \\
0 & -1
\end{array}\right)+\sum_{i} \frac{f^{2 i}(z)}{(2 i) !}\left(\begin{array}{ll}
1 & 0 \\
0 & 1
\end{array}\right) \\
& =\left(\begin{array}{cc}
\sum_{i} \frac{f^{i}(z)}{i !} & 0 \\
0 & \sum_{i} \frac{f^{i}(z)(-1)^{i}}{i !}
\end{array}\right) \\
& =\left(\begin{array}{cc}
e^{f(z)} & 0 \\
0 & e^{-f(z)}
\end{array}\right),
\end{aligned}
$$

where we used the properties of Pauli matrices. Consequently, by the asymptotic expression of $Y_{N}$, the asymptotic at infinity of $T_{N}$ can be obtained as follows:

$$
\begin{aligned}
T_{N}(z) & =e^{-\frac{N l}{2} \sigma_{3}}\left(\begin{array}{cc}
z^{N}+O\left(z^{n-1}\right) & O\left(z^{-n-1}\right) \\
O\left(z^{n-1}\right) & z^{-N}+O\left(z^{-n-1}\right)
\end{array}\right) e^{-N\left[g(z)-\frac{l}{2}\right] \sigma_{3}} \\
& =e^{-\frac{N l}{2} \sigma_{3}}\left(I+O\left(z^{-1}\right)\right)\left(\begin{array}{cc}
z^{N} & 0 \\
0 & z^{-N}
\end{array}\right) e^{-N\left[g(z)-\frac{l}{2}\right] \sigma_{3}} \\
& =\left(e^{-\frac{N l}{2} \sigma_{3}}+e^{-\frac{N l}{2} \sigma_{3}} O\left(z^{-1}\right)\right)\left(\begin{array}{cc}
z^{N} & 0 \\
0 & z^{-N}
\end{array}\right) e^{-N\left[g(z)-\frac{l}{2}\right] \sigma_{3}} \\
& =\left(I+O\left(z^{-1}\right)\right) e^{-\frac{N l}{2} \sigma_{3}}\left(\begin{array}{cc}
z^{N} & 0 \\
0 & z^{-N}
\end{array}\right) e^{-N\left[g(z)-\frac{l}{2}\right] \sigma_{3}} \\
& =\left(I+O\left(z^{-1}\right)\right)\left(\begin{array}{cc}
z^{N} & 0 \\
0 & z^{-N}
\end{array}\right) e^{-N g(z) \sigma_{3}}
\end{aligned}
$$

In addition, for our purpose we assume at infinity that

$$
e^{-N g(z) \sigma_{3}}=\left(\begin{array}{cc}
z^{-N} & 0 \\
0 & z^{N}
\end{array}\right)+O\left(z^{-1}\right),
$$

more precisely, for normalizing the asymptotic condition we have to assume 
Chapter 6. Large asymptotics of orthogonal polynomials: The

$$
\left(\begin{array}{cc}
e^{-N g(z)} & 0 \\
0 & e^{N g(z)}
\end{array}\right)=\left(\begin{array}{cc}
e^{-N \log z} & 0 \\
0 & e^{N \log z}
\end{array}\right)+O\left(z^{-1}\right) .
$$

Observe that $g$ has to satisfy, $g(z)=\log z+O\left(z^{-1}\right)$, as $z \rightarrow \infty$. Therefore, inserting (6.3) into (6.2) we obtain

$$
T_{N}(z)=\left(I+O\left(z^{-1}\right)\right)\left(\begin{array}{cc}
z^{N} & 0 \\
0 & z^{-N}
\end{array}\right)\left(\begin{array}{cc}
z^{-N} & 0 \\
0 & z^{N}
\end{array}\right)=I+O\left(z^{-1}\right) .
$$

So long as $g$ can have jump on the real axis, by the transformation (6.1) the

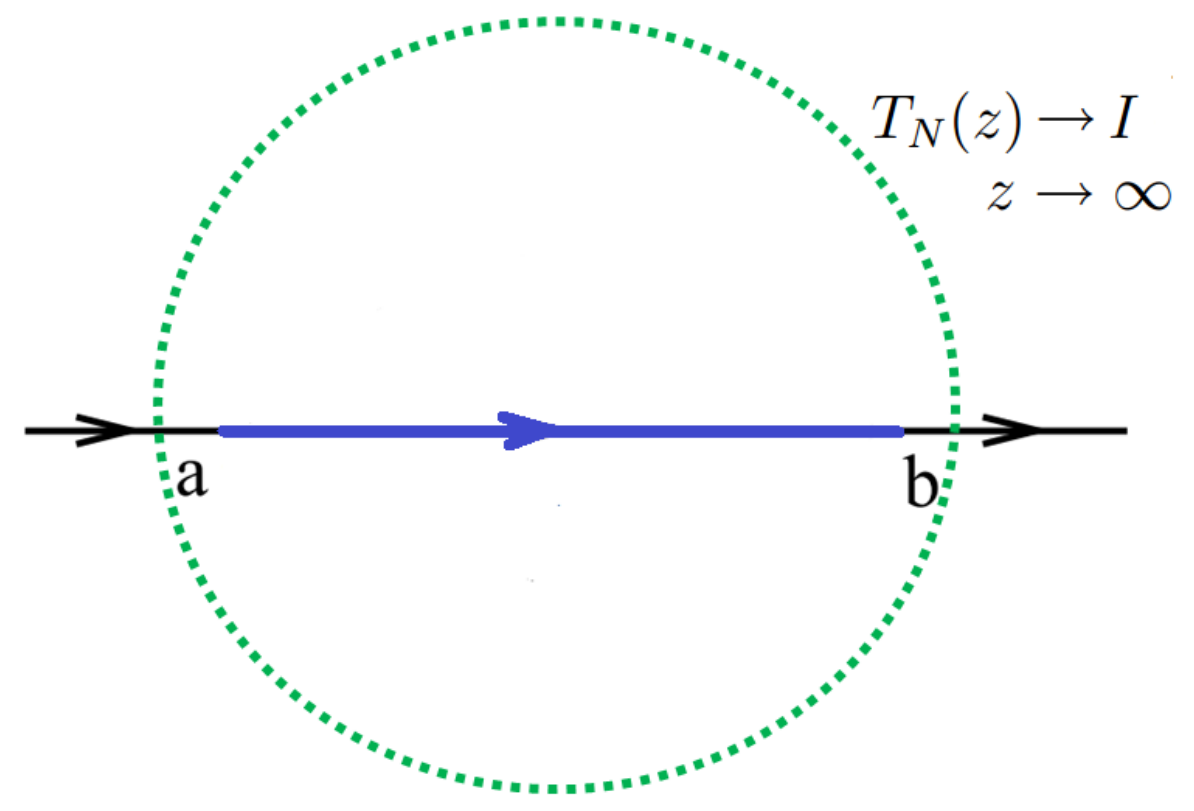

Figure 6.1: Goal of the first transformation $T_{N}$ : normalize the asymptotic condition of the RHP for the orthogonal polynomials.

jump matrix in the RHP for $Y_{N}$ is modified in the following way

$$
T_{N+}(x)=e^{-\frac{N l}{2} \sigma_{3}} Y_{N+}(x) e^{-N\left[g_{+}(x)-\frac{l}{2}\right] \sigma_{3}} .
$$

In fact, using the jump of $Y_{N}$ we have

$$
T_{N+}(x)=e^{-\frac{N l}{2} \sigma_{3}} Y_{N-}(x)\left(\begin{array}{cc}
1 & e^{-N V(x)} \\
0 & 1
\end{array}\right) e^{-N\left[g_{+}(x)-\frac{l}{2}\right] \sigma_{3}} .
$$

Inserting, $I=e^{-N\left[g_{-}(x)-\frac{l}{2}\right] \sigma_{3}} e^{N\left[g_{-}(x)-\frac{l}{2}\right] \sigma_{3}}$, in the preceding expression

$$
\begin{aligned}
T_{N+}(x)= & e^{-\frac{N l}{2} \sigma_{3}} Y_{N-}(x) e^{-N\left[g_{-}(x)-\frac{l}{2}\right] \sigma_{3}} e^{N\left[g_{+}(x)-\frac{l}{2}\right] \sigma_{3}}\left(\begin{array}{cc}
1 & e^{-N V(x)} \\
0 & 1
\end{array}\right) \times \\
& \times e^{-N\left[g_{+}(x)-\frac{l}{2}\right] \sigma_{3}},
\end{aligned}
$$


Chapter 6. Large asymptotics of orthogonal polynomials: The

as well as identifying, $T_{N-}(x)=e^{-\frac{N l}{2} \sigma_{3}} Y_{N-}(x) e^{-N\left[g_{-}(x)-\frac{l}{2}\right] \sigma_{3}}$, we obtain

$$
T_{N+}(x)=T_{N-}(x) e^{N\left[g_{-}(x)-\frac{l}{2}\right] \sigma_{3}}\left(\begin{array}{cc}
1 & e^{-N V(x)} \\
0 & 1
\end{array}\right) e^{-N\left[g_{+}(x)-\frac{l}{2}\right] \sigma_{3}} .
$$

Therefore, symplifying the jump matrix we show

$$
T_{N+}(x)=T_{N-}(x)\left(\begin{array}{cc}
e^{-N\left[g_{+}(x)-g_{-}(x)\right]} & e^{N\left[g_{+}(x)+g_{-}(x)-V(x)-l\right]} \\
0 & e^{N\left[g_{+}(x)-g_{-}(x)\right]}
\end{array}\right) .
$$

As the transformation (6.1) does not change the first condition of the RHP for $Y_{N}$. Then $T_{N}(z)$ solves uniquely the following RHP:

1. $T_{N}$ is analytic in $\mathrm{C} \backslash \mathrm{R}$,

2. $T_{N+}(x)=T_{N-}(x) j_{T}(x), x \in \mathrm{R}$, where

$$
j_{T}(x)=\left(\begin{array}{cc}
e^{-N\left[g_{+}(x)-g_{-}(x)\right]} & e^{N\left[g_{+}(x)+g_{-}(x)-V(x)-l\right]} \\
0 & e^{N\left[g_{+}(x)-g_{-}(x)\right]}
\end{array}\right)
$$

3. $T_{N}(z)=I+O\left(z^{-1}\right), z \rightarrow \infty$.

Furthermore, if we want $j_{T} \rightarrow I$ as $N \rightarrow \infty$, we consider that $g$ satisfies the following conditions

$$
\begin{gathered}
g_{+}(x)-g_{-}(x)=2 k \pi i, k \in Z, x \in \mathrm{R} \\
g_{+}(x)+g_{-}(x)-V(x)-l<0, x \in \mathrm{R},
\end{gathered}
$$

thereupon the jump matrix $j_{T}$ has the following asymptotic behaviour as $N \rightarrow \infty$

$$
j_{T}(x)=\left(\begin{array}{cc}
e^{-2 N k \pi i} & e^{-N c(x)} \\
0 & e^{2 N k \pi i}
\end{array}\right)=\left(\begin{array}{ll}
1 & 0 \\
0 & 1
\end{array}\right),
$$

where $c(x)>0$. Observe that $g$ is related to the asymptotic behaviour of $T_{N}$ and $j_{T}$, for this reason we will determine all properties of $g$. First from (6.3) without loss of generality we have that

$$
e^{N g(z)} \approx p_{N}(z)
$$

where $p_{N}(z)$ is an orthogonal polynomial of degree $N$. Hence by the Heine formula [17], $p_{N}(z)$ is the average difference of the diagonal elements in the random matrix with respect to $z$, we can write that

$$
e^{N g(z)}=\langle\operatorname{det}(z-M)\rangle .
$$

Certainly, in the ensemble of eigenvalues we have 
Chapter 6. Large asymptotics of orthogonal polynomials: The

$e^{N g(z)}=\left\langle\Pi_{j=1}^{N}\left(z-\lambda_{j}\right)\right\rangle=\frac{1}{Z_{N}} \int \Pi_{j=1}^{N}\left(z-\lambda_{j}\right) \Pi_{j<k}\left(\lambda_{j}-\lambda_{k}\right)^{2} \Pi_{j=1}^{N} e^{-N V\left(\lambda_{j}\right)} d \lambda_{j}$

Therefore, due to the Heine formula we can associate $g$ to the equilibrium measure of the eigenvalues of the following way

$$
g(z)=\frac{1}{N} \log \left\langle\Pi_{j=1}^{N}\left(z-\lambda_{j}\right)\right\rangle=\int_{J} \log (z-x) d \nu_{V}(x),
$$

where for obtaining the other properties of $g$ we assume that the equilibrium measure $\nu_{V}$ is regular and we take the principal branch for logarithm. Second, we will study the asymptotic behavior of $g$, which was established before for our convenience. Formally,

$$
\begin{aligned}
g(z) & =\int_{J} \log z \log \left(1-\frac{x}{z}\right) d \nu_{V}(x) \\
& =\int_{J} \log z d \nu_{V}(x)+\int_{J} \log \left(1-\frac{x}{z}\right) d \nu_{V}(x) \\
& =\log z \int_{J} d \nu_{V}(x)+\int_{J} \log \left(1-\frac{x}{z}\right) d \nu_{V}(x) .
\end{aligned}
$$

Upon the condition that $J$ is a finite union of intervals, for $z \rightarrow \infty$ we can expand the logarithm in the following way

$$
g(z)=\log z-\int_{J} \sum_{j=1}^{\infty} \frac{\left(\frac{x}{z}\right)^{j}}{j} d \nu_{V}(x)
$$

where we substituted $\int_{J} d \nu_{V}(x)=1$. As can be seen the asymptotic behavior of $g$ as $z \rightarrow \infty$ is given by

$$
g(z)=\log z-\sum_{j=1}^{\infty} \frac{g_{j}}{z^{j}} d \nu_{V}(x)=\log z+O\left(z^{-1}\right), g_{j}=\int_{J} \frac{x^{j}}{j} d \nu_{V}(x)
$$

which guarantees the normalization of the asymptotic condition of the RHP. Third, we will analize the jumps of $g$ on the real axis, which were considered before for ensuring the asymptotic behavior of the jump matrix $j_{T}$. As has been noted, $\log z$ has a jump on the negative real axis. So for the principal branch of the logarithm a circuit in the clockwise direction put us on the branch $\log z=\log |z|-\pi i$ while in the counterclokwise direction put us on the branch $\log z=\log |z|+\pi i$. Under those circumstances, we can generalize the jumps on the following way

$$
\log (x-y)_{ \pm}=\left\{\begin{array}{cc}
\log |x-y| & x>y \\
\log |x-y| \pm \pi i & x<y
\end{array}\right.
$$


Chapter 6. Large asymptotics of orthogonal polynomials: The

hence from (6.17) this implies that

$$
\begin{aligned}
g_{ \pm}(x) & =\int_{J} \log (x-y)_{ \pm} d \nu_{V}(y) \\
& =\int_{J}[\log |x-y| \pm \pi i \theta(y-x)] d \nu_{V}(y) \\
& =\int_{J} \log |x-y| d \nu_{V}(y) \pm \pi i \int_{J} \theta(y-x) d \nu_{V}(y)
\end{aligned}
$$

By manipulying the preceding expressions to compare with (6.11) and (6.12) we obtain that

$$
\begin{aligned}
& g_{+}(x)-g_{-}(x)=2 \pi i \int_{J} \theta(y-x) d \nu_{V}(y) \\
& g_{+}(x)+g_{-}(x)=2 \int_{J} \log |x-y| d \nu_{V}(y) .
\end{aligned}
$$

Observe that from the equation (6.23) we have that the function

$$
G(x)=g_{+}(x)-g_{-}(x)
$$

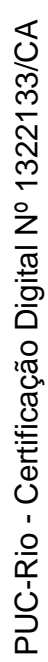

is pure imaginary for all real $x$, and we also have that

$$
G(x)=2 \pi i, x \in \mathrm{R} \backslash J,
$$

because $\int_{J} d \nu_{V}(y)=1$. Furthermore, the equation (6.24) can be related to

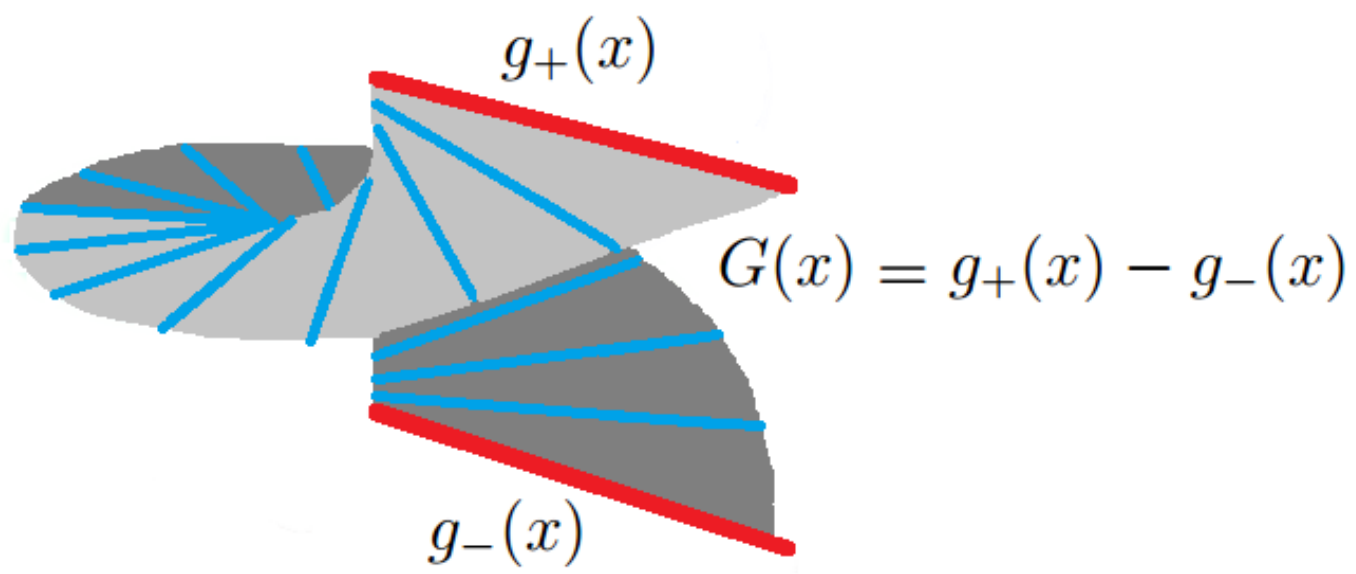

Figure 6.2: Analytic definition(continuation) of $G$.

$V(x)$ by the Euler-Lagrange variational conditions [17]. Consequently for the same constant $l$, we obtain

$$
\begin{gathered}
g_{+}(x)+g_{-}(x)=2 \int_{J} \log |x-y| d \nu_{V}(y)<V(x)+l, x \in \mathrm{R} \backslash J \\
g_{+}(x)+g_{-}(x)=2 \int_{J} \log |x-y| d \nu_{V}(y)=V(x)+l, x \in J .
\end{gathered}
$$

Observe that (6.23) and (6.27) coincide with the conditions (6.11) and (6.12) respectivaly. Therefore the above properties of $g$ guarantee the asymptotic 
Chapter 6. Large asymptotics of orthogonal polynomials: The

behavior of the jump matrix $j_{T}$ outside $J$. In particular the property (6.28) ensures that on $J$ we have

$$
j_{T}(x)=\left(\begin{array}{cc}
e^{-N G(x)} & 1 \\
0 & e^{N G(x)}
\end{array}\right) .
$$

In summary, the normalization of the asymptotic behavior was obtained after

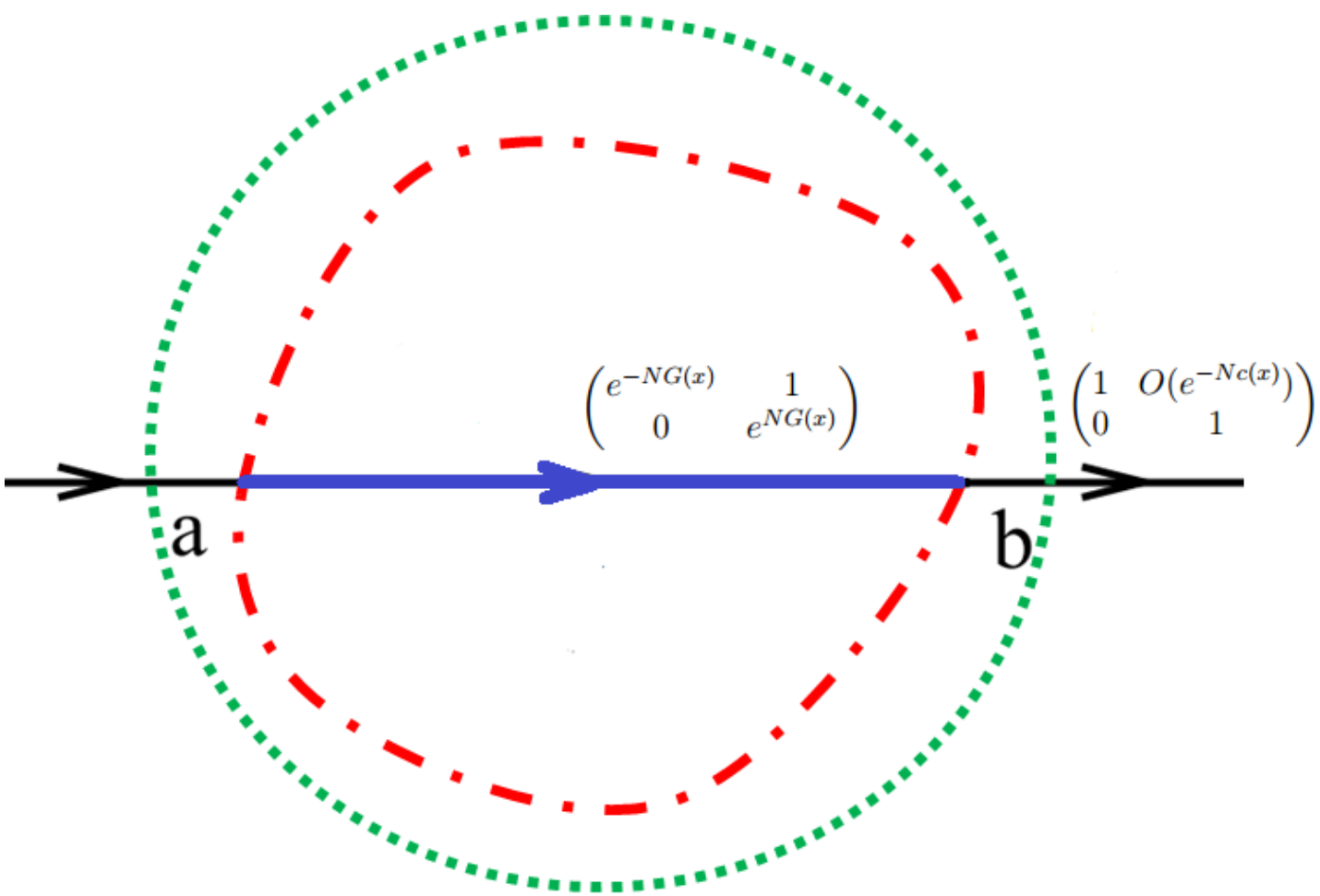

Figure 6.3: After the first transformation $T_{N}$ the jump condition was modified.

the first transformation but jump matrix was modified, and we observe that the jump oscillates over $x$. The Heine formula had sustained the asymptotic behavior of $T_{N}$ and $j_{T}$, through the function $g$.

\section{3}

\section{Second transformation of the RHP}

The first transformation changes the behavior of the jump matrix $j_{T}$, now this jump matrix has oscillatory behavior because $G$ is pure imaginary. The procedure is splitting the jump matrix by its factorization, and after extending analytically this jump matrix factors into several curves such that behavior of the factors approach to the identity for large $N$. Then we will demostrate this analytic extension. We assume that $J$ consists of a single interval $[a, b]$, by the 
Chapter 6. Large asymptotics of orthogonal polynomials: The

definition of the equilibrium mesure we have that

$$
\begin{aligned}
G(x) & =2 \pi i \int_{J=[a, b]} \theta(y-x) \frac{1}{2 \pi i} h(y){\sqrt{(y-a)(y-b)_{+}}} d y \\
& =\int_{x}^{b} h(y) \sqrt{(y-a)(y-b)_{+}} d y
\end{aligned}
$$

As $e^{ \pm N G(z)}$ is a continnous function and the integral along the contour that contains $[a, b]$ in its interior is given by

$$
\begin{aligned}
\oint e^{ \pm N G(z)} d z & =\int_{a}^{b} e^{ \pm N G(x)} d x-\int_{b}^{a} e^{ \pm N G(x)} d x \\
& =2 \int_{a}^{b} e^{ \pm N G(x)} d x \\
& =2 \int_{a}^{b} \sum_{j} \frac{( \pm N G(z))^{j}}{j !} d z \\
& =2 \int_{a}^{b}\left( \pm N G(x)+\sum_{j=1}^{\infty} \frac{( \pm N G(x))^{j}}{j !} d x\right. \\
& = \pm 2 N \int_{a}^{b} G(x) d x+2 \int_{a}^{b} \sum_{j=1}^{\infty} \frac{( \pm N G(x))^{j}}{j !} d x \\
& = \pm 2 N \int_{a}^{b} G(x) d x+2 \sum_{j=1}^{\infty} \int_{a}^{b} \frac{( \pm N G(x))^{j}}{j !} d x \\
\oint e^{ \pm N G(z)} d z & \neq 0,
\end{aligned}
$$

then as the integral is different from 0, by Morera's Theorem we have that $e^{ \pm N G(z)}$ is analytic in $\mathrm{C} \backslash[a, b]$. Accordingly, $e^{ \pm N G(z)}$ has analytic extension to $\mathrm{C} \backslash[a, b]$. Furthermore, by matrix algebra

$$
\left(\begin{array}{cc}
e^{a} & 1 \\
0 & e^{-a}
\end{array}\right)=\left(\begin{array}{cc}
1 & 0 \\
e^{-a} & 1
\end{array}\right)\left(\begin{array}{cc}
0 & 1 \\
-1 & 0
\end{array}\right)\left(\begin{array}{cc}
1 & 0 \\
e^{a} & 1
\end{array}\right)
$$

the matrix jump can be factorized as follows

$$
\left(\begin{array}{cc}
e^{-N G(x)} & 1 \\
0 & e^{N G(x)}
\end{array}\right)=\left(\begin{array}{cc}
1 & 0 \\
e^{N G(x)} & 1
\end{array}\right)\left(\begin{array}{cc}
0 & 1 \\
-1 & 0
\end{array}\right)\left(\begin{array}{cc}
1 & 0 \\
e^{-N G(x)} & 1
\end{array}\right),
$$

hence the contour equation for $T_{N}$ can now be written as

$$
T_{+}(x)=T_{-}(x)\left(\begin{array}{cc}
1 & 0 \\
e^{N G(x)} & 1
\end{array}\right)\left(\begin{array}{cc}
0 & 1 \\
-1 & 0
\end{array}\right)\left(\begin{array}{cc}
1 & 0 \\
e^{-N G(x)} & 1
\end{array}\right) .
$$

Eventually, by bringing the rightmost matrix to the left-hand side, we obtain 
Chapter 6. Large asymptotics of orthogonal polynomials: The

$$
\begin{aligned}
& T_{+}(x)\left(\begin{array}{cc}
1 & 0 \\
e^{-N G(x)} & 1
\end{array}\right)^{-1}=T_{-}(x)\left(\begin{array}{cc}
1 & 0 \\
e^{N G(x)} & 1
\end{array}\right)\left(\begin{array}{cc}
0 & 1 \\
-1 & 0
\end{array}\right) \\
& T_{+}(x)\left(\begin{array}{cc}
1 & 0 \\
-e^{-N G(x)} & 1
\end{array}\right)=T_{-}(x)\left(\begin{array}{cc}
1 & 0 \\
e^{N G(x)} & 1
\end{array}\right)\left(\begin{array}{cc}
0 & 1 \\
-1 & 0
\end{array}\right),
\end{aligned}
$$

where we can define the nonconstant matrices, $j_{ \pm}(x)=\left(\begin{array}{cc}1 & 0 \\ \mp e^{\mp N G(x)} & 1\end{array}\right)$, such that the contour equation is given by

$$
T_{+}(x) j_{+}(x)=T_{-}(x) j_{-}(x)\left(\begin{array}{cc}
0 & 1 \\
-1 & 0
\end{array}\right) .
$$

Now, we will define the analytic extension of $j_{ \pm}(x)$ such that this extension approaches to the identity for large $N$. To do so we rewrite the equation (6.30) in the following way

$$
G(x)=-i \int_{b}^{x} h(y) \sqrt{(y-a)(b-y)}+d y
$$

hence the analytic extension of this expression is given by

$$
G(z)=-i \int_{b}^{z} h(y) \sqrt{(y-a)(b-y)} d y,
$$

where $z=x+i w$. In fact, the preceding expression is an analytic continuation to the upper half plane $\mathrm{C}^{+}$. Then on one hand the derivative of $G(z)$ yields

$$
\frac{d}{d z} G(z)=-i h(z) \sqrt{(z-a)(b-z)}
$$

and on the other hand the Cauchy-Riemann equations give

$$
\frac{d}{d z} G(z)=\frac{\partial}{\partial y} \operatorname{Im} G(z)-i \frac{\partial}{\partial y} \operatorname{Re} G(z) .
$$

Thus by comparing both expression one gets

$$
\frac{\partial}{\partial y} \operatorname{Re} G(z)=h(z) \sqrt{(z-a)(b-z)}
$$

Due to $\sqrt{(z-a)(b-z)}>0$ and $h(z)>0$, one finds that

$$
\frac{\partial}{\partial y} \operatorname{Re} G(z)>0 \text { for } \operatorname{Im} z>0 \text {. }
$$

Therefore $e^{-N G(z)}$ decays exponentially on the upper half plane $\mathrm{C}^{+}$. Hence for this reason exists $c>0$ such that

$$
\left|e^{-N G(z)}\right| \leq e^{-N c}, z \in R^{+}=\{z=x+i y / a<x<b, 0<y\}
$$

which ensures that $e^{-N G(x)}$ admits analytic continuation in the upper half plane $\mathrm{C}^{+}$. Also owing to $\left|e^{N G(z)}\right|=\left|e^{-N G(\bar{z})}\right|$, exist $c>0$ such that 
Chapter 6. Large asymptotics of orthogonal polynomials: The Riemann-Hilbert approach

$$
\left|e^{N G(z)}\right| \leq e^{-N c}, z \in R^{-}=\{z=x+i y / a<x<b, y<0\}
$$

In the same way $e^{N G(x)}$ admits analytic continuation in the lower half plane $\mathrm{C}^{-}$. As a result the matrices $j_{ \pm}(x)$ accept analytic continuation in the upper/lower half planes $\mathrm{C}^{ \pm}$respectively. Furthermore, we consider the curves $\gamma^{ \pm} \subset R^{ \pm}$

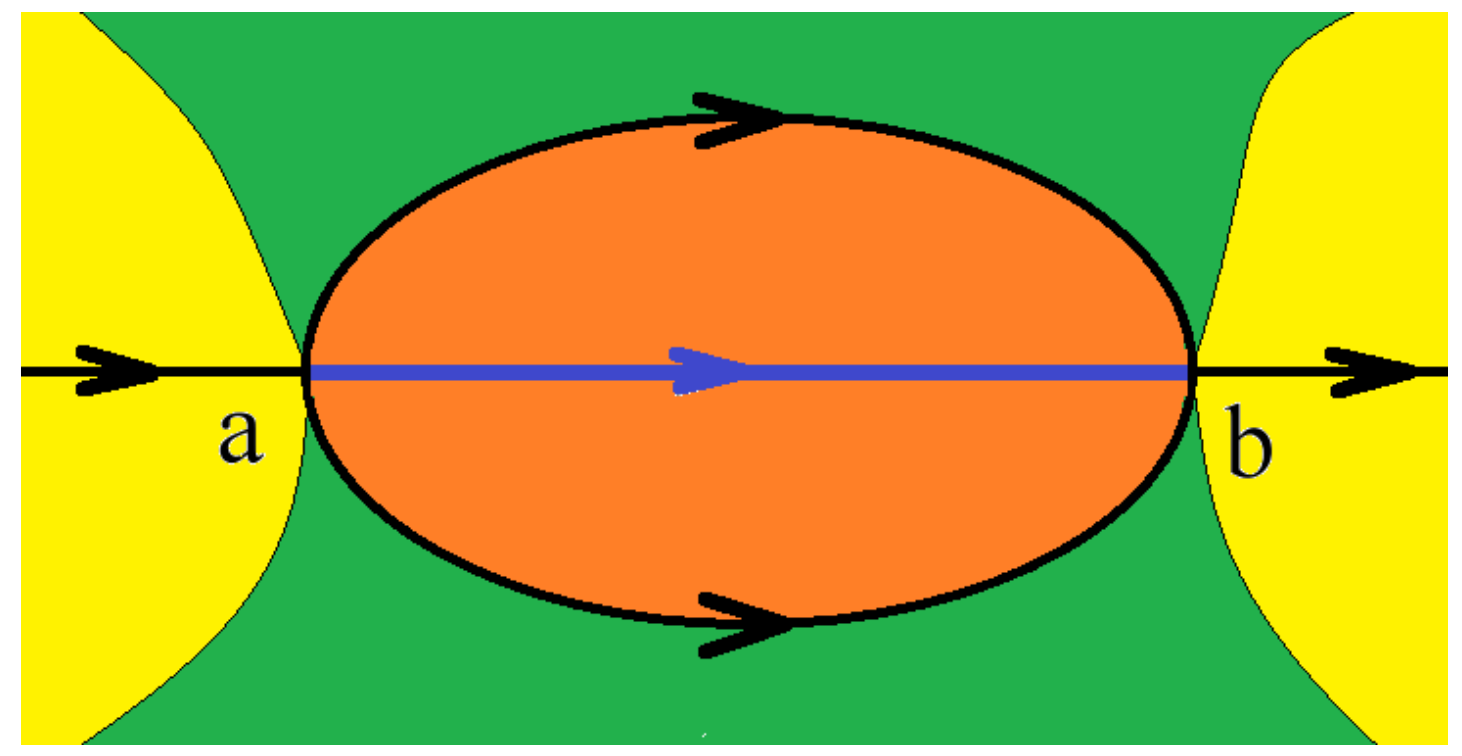

Figure 6.4: Analytic continuation of the contours for the jump condition of $T_{N}$. Where quantitatibly the different coulors correspond to different solutions of the RHP for $T_{N}$ such that for large $N$, the yellow and green regions will became only one region.

such that the region bounded by the interval $[a, b]$ and $\gamma^{+} / \gamma^{-}$is called the upper/lower lens $\mathcal{L}^{ \pm}$, respectively. Here we observe that the curves $\gamma^{ \pm}$ correspond to contour deformation. Then, this allows us to define the following deformation of $T_{N}$

$$
S_{N}=\left\{\begin{array}{cc}
T_{N}(z) j_{+}(z) & z \in \mathcal{L}^{+} \\
T_{N}(z) j_{-}(z) & z \in \mathcal{L}^{-} \\
T_{N}(z) & \text { otherwise }
\end{array}\right.
$$

where $j_{ \pm}$approach to the identity for large $N$. Thereupon, by the transformation (6.45) the jump matrix in the RHP for $T_{N}$ is modified in the following way: First of all, by the contour equation (6.36) on the support $z \in[a, b]$ we get

$$
T_{N+}(z) j_{+}(z)=T_{N-}(z) j_{-}(z)\left(\begin{array}{cc}
0 & 1 \\
-1 & 0
\end{array}\right),
$$

through the definition of $S_{N}$ we have

$$
S_{N+}(z)=S_{N-}(z)\left(\begin{array}{cc}
0 & 1 \\
-1 & 0
\end{array}\right) .
$$

Second, again via the definition of $S_{N}$ on the upper lens $\gamma^{+}$we have 
Chapter 6. Large asymptotics of orthogonal polynomials: The Riemann-Hilbert approach

$$
S_{N+}(z)=T_{N+}(z)\left(j_{+}(z)\right)_{+}
$$

taking into account that $\left(j_{+}(z)\right)_{+}$decays exponentially to $I$ we find

$$
S_{N+}(z)=T_{N+}(z)=T_{N}(z)\left(\begin{array}{cc}
1 & 0 \\
0 & 1
\end{array}\right)
$$

by introducing, $\left(\begin{array}{ll}1 & 0 \\ 0 & 1\end{array}\right)=\left(\begin{array}{cc}1 & 0 \\ e^{N G(z)} & 1\end{array}\right)\left(\begin{array}{cc}1 & 0 \\ -e^{N G(z)} & 1\end{array}\right)$, we get

$$
S_{N+}(z)=T_{N}(z)\left(\begin{array}{cc}
1 & 0 \\
e^{N G(z)} & 1
\end{array}\right)\left(\begin{array}{cc}
1 & 0 \\
-e^{N G(z)} & 1
\end{array}\right),
$$

hence, according to the definition of $S_{N-}$ we find

$$
S_{N+}(z)=S_{N-}(z)\left(\begin{array}{cc}
1 & 0 \\
-e^{N G(z)} & 1
\end{array}\right),
$$

but bearing in mind (6.43) we obtain

$$
S_{N+}(z)=S_{N-}(z)\left(\begin{array}{cc}
1 & 0 \\
O\left(e^{-c N}\right) & 1
\end{array}\right), z \in \gamma^{+}
$$

Analogously for the lower lens $\gamma^{-}$we obtain

$$
S_{N+}(z)=S_{N-}(z)\left(\begin{array}{cc}
1 & 0 \\
O\left(e^{-c N}\right) & 1
\end{array}\right), z \in \gamma^{-} .
$$

And finally the jump matrix is the same outside the support $[a, b]$,

$$
j_{S}(z)=j_{T}(z), z \in \mathrm{R} \backslash[a, b] .
$$

Therefore $S_{N}$ solves uniquely the following RHP:

1. $S_{N}$ is analytic in $\mathrm{C} \backslash\left(\mathrm{R} \cup \gamma^{+} \cup \gamma^{-}\right)$,

2.

$$
S_{N+}(x)=S_{N-}(x) j_{S}(x), x \in \mathrm{R} \cup \gamma^{-} \cup \gamma^{+},
$$

such that the jump matrices are given by

a.

$$
j_{S}(x)=\left(\begin{array}{cc}
0 & 1 \\
-1 & 0
\end{array}\right), z \in[a, b]
$$

b.

$$
j_{S}(x)=\left(\begin{array}{cc}
1 & 0 \\
O\left(e^{-c N}\right) & 1
\end{array}\right), z \in \gamma^{ \pm}
$$


Chapter 6. Large asymptotics of orthogonal polynomials: The Riemann-Hilbert approach

c.

$$
j_{S}(x)=\left(\begin{array}{cc}
1 & O\left(e^{-c N}\right) \\
0 & 1
\end{array}\right), z \in \mathrm{R} \backslash[a, b]
$$

where $c>0$

3. $S_{N}(z)=I+O\left(z^{-1}\right), z \rightarrow \infty$.

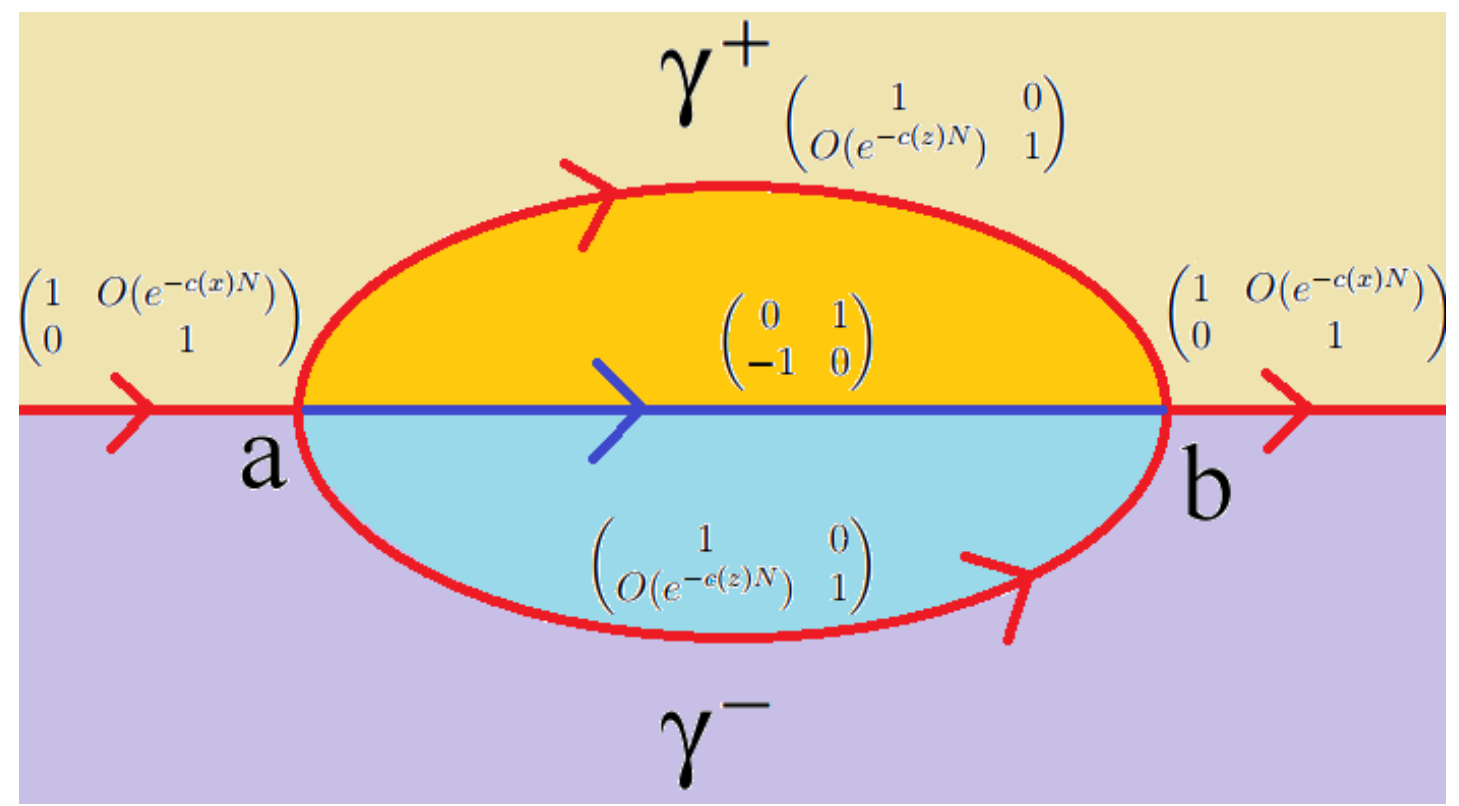

Figure 6.5: After the second transformation $S_{N}$ only the jump condition in the interval $[a, b]$ did not tend to the identity when $N \rightarrow \infty$.

Observe that the only jump matrix which is not close to the identity is the jump on $[a, b]$. While the other jumps, on $\mathrm{R} \cup \gamma^{+} \cup \gamma^{-} \backslash[a, b]$, have only one element off-diagonal that tends exponentially to zero except in the edge points $a$ and $b$, where the jump matrix is given by

$$
\begin{gathered}
j_{S}(a, b)=\left(\begin{array}{ll}
1 & 0 \\
1 & 1
\end{array}\right), a, b \in \overline{\gamma^{ \pm}} \\
j_{S}(a, b)=\left(\begin{array}{ll}
1 & 1 \\
0 & 1
\end{array}\right), a, b \in \overline{\mathrm{R} \backslash[a, b]}
\end{gathered}
$$

This problem at the edge points can be resolved if one deforms the edge points to small disks. Thence, the RHP can be solved explicity inside this disks where the local solution is called parametrix.

In the generic cases, this parametrix can be constructed with the aid of Airy functions but in nongeneric situations one could need other especial functions. Meanwhile, If we ignore for this moment the parametrix at the edge points and we drop the terms of ordem $O\left(e^{-c N}\right)$ in the jump matrix $j_{S}(z)$, we obtain the following model RHP: 
Chapter 6. Large asymptotics of orthogonal polynomials: The Riemann-Hilbert approach

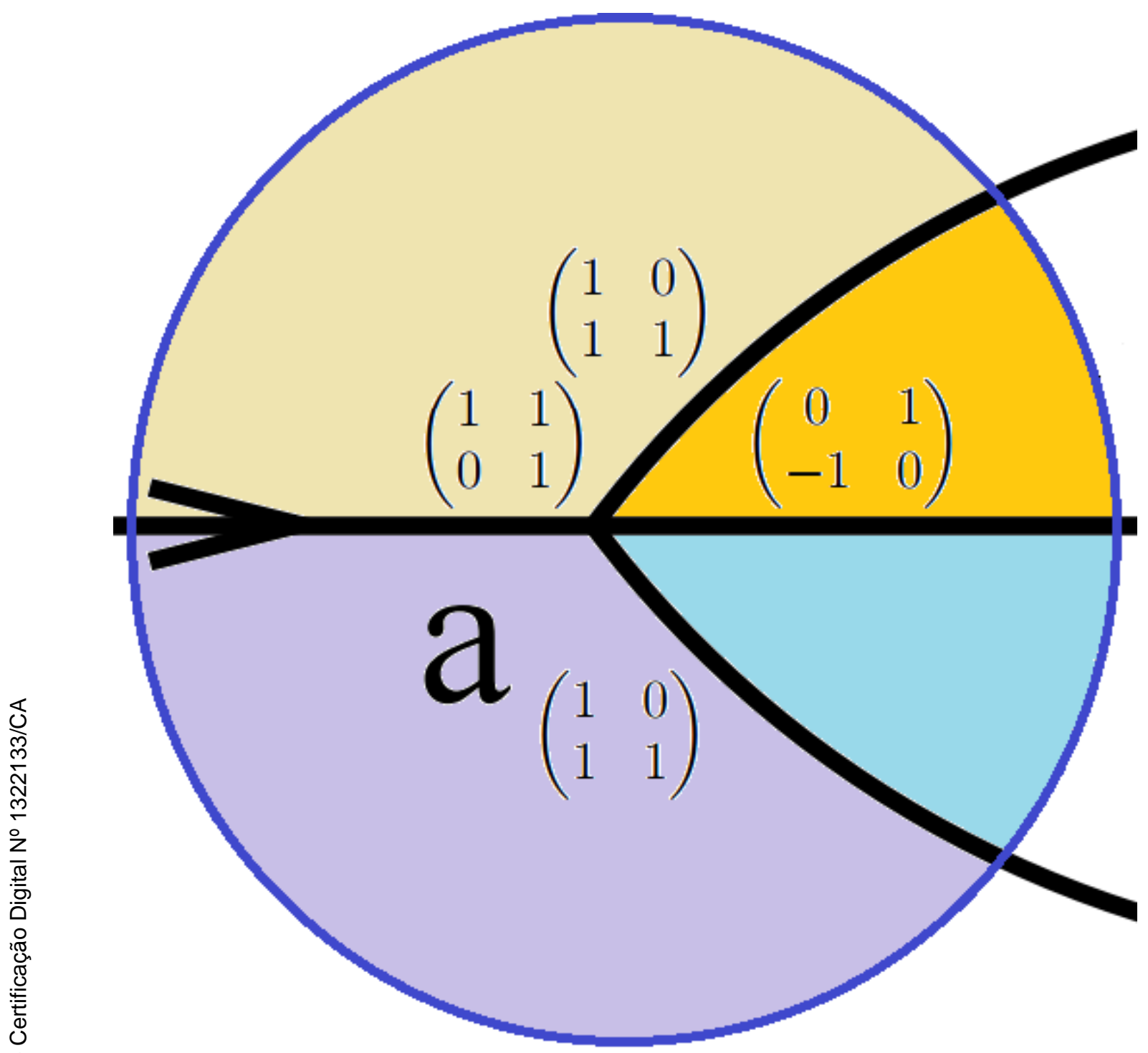

Figure 6.6: Problem in the edge point $a$. In this point the jump matrix has a bad behavior by the intersection of four contours.

1. $\mathrm{M}(\mathrm{z})$ is analytic in $\mathrm{C} \backslash[a, b]$,

2. $M_{+}(z)=M_{-}(z)\left(\begin{array}{cc}0 & 1 \\ -1 & 0\end{array}\right), z \in R$

3. $M(z)=I+O\left(z^{-1}\right), z \rightarrow \infty$.

we can diagonalize the jump matrix of this model RHP

$$
\left(\begin{array}{cc}
0 & 1 \\
-1 & 0
\end{array}\right)=\frac{1}{2}\left(\begin{array}{cc}
1 & 1 \\
i & -i
\end{array}\right)\left(\begin{array}{cc}
i & 0 \\
0 & -i
\end{array}\right)\left(\begin{array}{cc}
1 & -i \\
1 & i
\end{array}\right)
$$

by replacing in the jump condition, we have

$$
M_{+}(z)=M_{-}(z) \frac{1}{2}\left(\begin{array}{cc}
1 & 1 \\
i & -i
\end{array}\right)\left(\begin{array}{cc}
i & 0 \\
0 & -i
\end{array}\right)\left(\begin{array}{cc}
1 & -i \\
1 & i
\end{array}\right) .
$$


Chapter 6. Large asymptotics of orthogonal polynomials: The

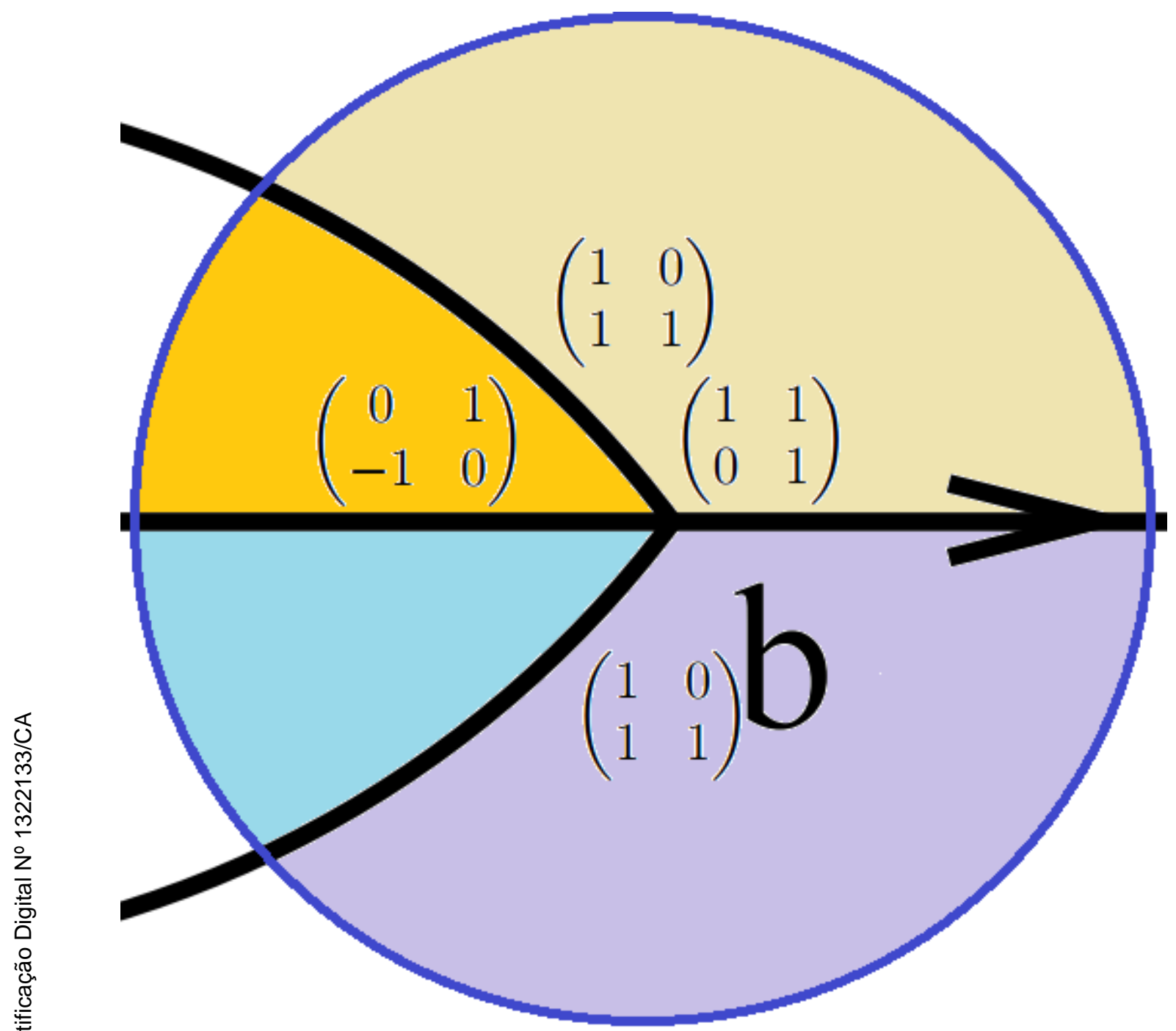

Figure 6.7: Problem in the edge point $b$. In the same way the jump matrix has a bad behavior by the intersection of four contours

Now we can reduce this RHP to a pair of scalar RHP through the following procedure:

First, by multiplying both sides of the jump condition(6.58) by $\left(\begin{array}{cc}1 & -i \\ 1 & i\end{array}\right)^{-1}$,

$$
M_{+}(z) \frac{1}{2}\left(\begin{array}{cc}
1 & 1 \\
i & -i
\end{array}\right)=M_{-}(z) \frac{1}{2}\left(\begin{array}{cc}
1 & 1 \\
i & -i
\end{array}\right)\left(\begin{array}{cc}
i & 0 \\
0 & -i
\end{array}\right)
$$

next, by multiplying both sides of the same jump condition by $\left(\begin{array}{cc}1 & -i \\ 1 & i\end{array}\right)$, we obtain

$\frac{1}{2}\left(\begin{array}{cc}1 & -i \\ 1 & i\end{array}\right) M_{+}(z)\left(\begin{array}{cc}1 & 1 \\ i & -i\end{array}\right)=\frac{1}{2}\left(\begin{array}{cc}1 & -i \\ 1 & i\end{array}\right) M_{-}(z)\left(\begin{array}{cc}1 & 1 \\ i & -i\end{array}\right)\left(\begin{array}{cc}i & 0 \\ 0 & -i\end{array}\right)$,

finally, notice that this modified jump condition suggests the following transformation 
Chapter 6. Large asymptotics of orthogonal polynomials: The

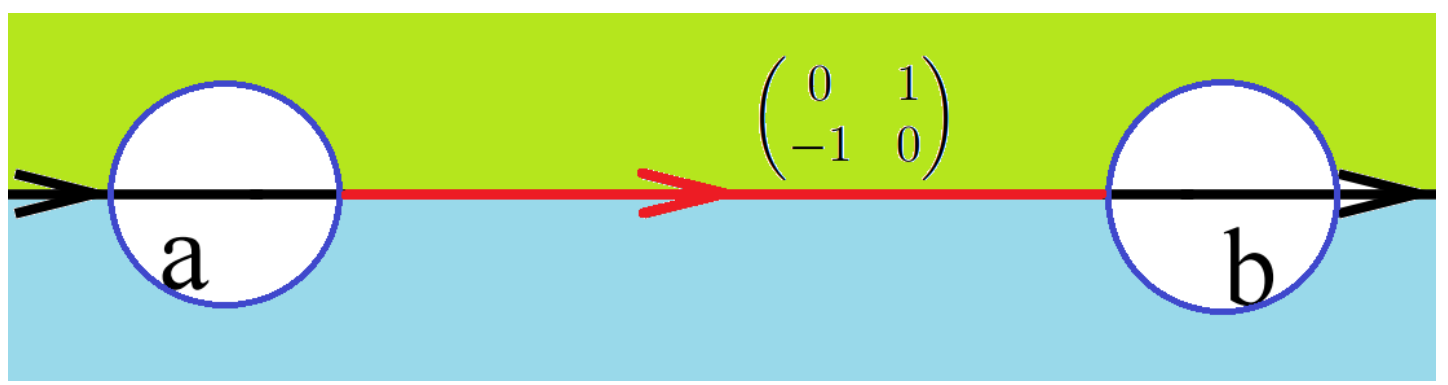

Figure 6.8: Representation of the RHP for M.

$$
\tilde{M}(z)=\frac{1}{2}\left(\begin{array}{cc}
1 & -i \\
1 & i
\end{array}\right) M(z)\left(\begin{array}{cc}
1 & 1 \\
i & -i
\end{array}\right)
$$

such that $\tilde{M}$ is the unique solution of the following RHP

1. $\tilde{M}$ is analytic in $\mathrm{C} \backslash[a, b]$,

2. $\tilde{M}_{+}(z)=\tilde{M}_{-}(z)\left(\begin{array}{cc}i & 0 \\ 0 & -i\end{array}\right), z \in R$,

3. $\tilde{M}(z)=I+O\left(z^{-1}\right), z \rightarrow \infty$.

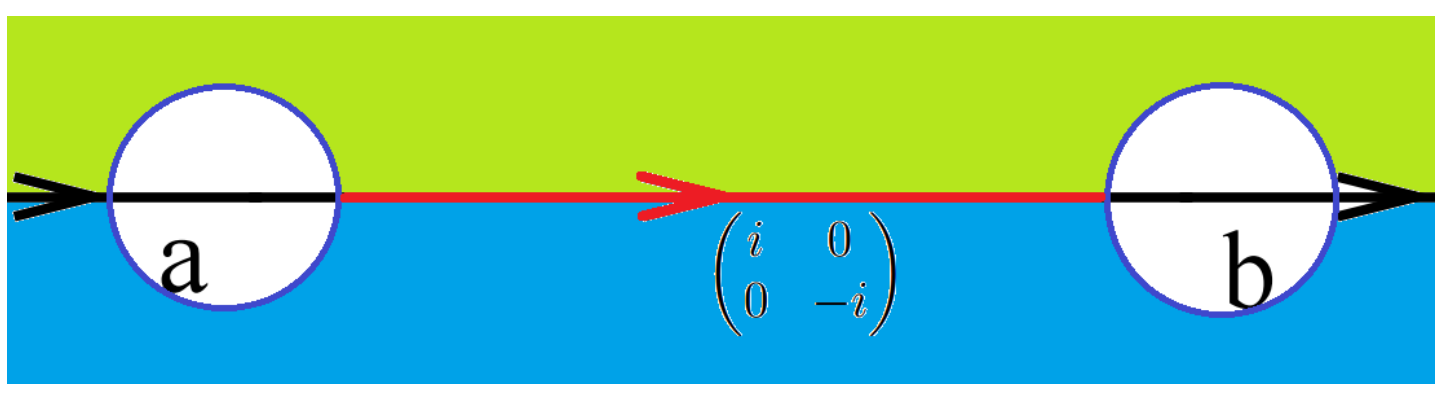

Figure 6.9: Representation of the RHP for $\tilde{M}$.

which corresponds to a pair of scalar RHP of the diagonal elements of $\tilde{M}$, so that this RHPs have unique solution as a Cauchy type integral in the diagonal elements of $\tilde{M}$, in the following way

$$
\begin{aligned}
\tilde{M}(z) & =\left(\begin{array}{cc}
e^{\frac{1}{2 \pi i} \int_{a}^{b} \frac{\log i}{s-z} d s} & 0 \\
0 & e^{\frac{1}{2 \pi i} \int_{a}^{b} \frac{\log (-i)}{s-z} d s}
\end{array}\right) \\
& =\left(\begin{array}{cc}
e^{\frac{1}{4} \log \frac{z-b}{z-a}} & 0 \\
0 & e^{-\frac{1}{4} \log \frac{z-b}{z-a}}
\end{array}\right) \\
& =\left(\begin{array}{cc}
\left(\frac{z-b}{z-a}\right)^{\frac{1}{4}} & 0 \\
0 & \left(\frac{z-a}{z-b}\right)^{\frac{1}{4}}
\end{array}\right),
\end{aligned}
$$


Chapter 6. Large asymptotics of orthogonal polynomials: The

tracing back to $\mathrm{M}$ by the inverse trasformation of (6.61), we have

$$
\begin{aligned}
M(z) & =\frac{1}{2}\left(\begin{array}{cc}
1 & 1 \\
i & -i
\end{array}\right)\left(\begin{array}{cc}
\left(\frac{z-b}{z-a}\right)^{\frac{1}{4}} & 0 \\
0 & \left(\frac{z-a}{z-b}\right)^{\frac{1}{4}}
\end{array}\right)\left(\begin{array}{cc}
1 & -i \\
1 & i
\end{array}\right) \\
& =\frac{1}{2}\left(\begin{array}{cc}
\left(\frac{z-b}{z-a}\right)^{\frac{1}{4}} & \left(\frac{z-a}{z-b}\right)^{\frac{1}{4}} \\
i\left(\frac{z-b}{z-a}\right)^{\frac{1}{4}} & -i\left(\frac{z-a}{z-b}\right)^{\frac{1}{4}}
\end{array}\right)\left(\begin{array}{cc}
1 & -i \\
1 & i
\end{array}\right) \\
& =\frac{1}{2}\left(\begin{array}{cc}
\left(\frac{z-b}{z-a}\right)^{\frac{1}{4}}+\left(\frac{z-a}{z-b}\right)^{\frac{1}{4}} & \left(-\left(\frac{z-b}{z-a}\right)^{\frac{1}{4}}+\left(\frac{z-a}{z-b}\right)^{\frac{1}{4}}\right) \\
i\left(\left(\frac{z-b}{z-a}\right)^{\frac{1}{4}}-\left(\frac{z-a}{z-b}\right)^{\frac{1}{4}}\right) & \left(\frac{z-b}{z-a}\right)^{\frac{1}{4}}+\left(\frac{z-a}{z-b}\right)^{\frac{1}{4}}
\end{array}\right) .
\end{aligned}
$$

To study the asymptotic behavior of $M(z)$ and compute $|M(z)|$, we define $\gamma(z) \equiv\left(\frac{z-b}{z-a}\right)^{\frac{1}{4}}$, thence the determinant of $M(z)$ is given by

$$
|M(z)|=\frac{1}{4}\left[\left(\gamma+\gamma^{-1}\right)^{2}-\left(\gamma-\gamma^{-1}\right)^{2}\right]=\frac{1}{4} 4 \gamma \gamma^{-1}=1
$$

and the asymptotic behavior of $\gamma(z)$ at infinity is obtained in the following way

$$
\begin{aligned}
\gamma(z) & =\left(\frac{z-b}{z-a}\right)^{\frac{1}{4}}=\left(\frac{z-b}{z-b}+\frac{b-a}{z-b}\right)^{\frac{1}{4}} \\
& =\left(1+\frac{b-a}{z-b}\right)^{\frac{1}{4}}=1+\frac{1}{4} \frac{b-a}{z}+O\left(z^{-2}\right)
\end{aligned}
$$

taking the inverse by the approximate Taylor expansion

$$
\gamma^{-1}(z)=1-\frac{1}{4} \frac{b-a}{z}+O\left(z^{-2}\right)
$$

therefore, substituting both asymptotic behaviors of $\gamma$ and $\gamma^{-1}$ in $M(z)$, we find

$$
\begin{aligned}
M(z) & =\frac{1}{2}\left(\begin{array}{cc}
2+O\left(z^{-2}\right) & i \frac{2(b-a)}{4 z}+O\left(z^{-2}\right) \\
-i \frac{2(b-a)}{4 z}+O\left(z^{-2}\right) & 2+O\left(z^{-2}\right)
\end{array}\right) \\
& =\left(\begin{array}{cc}
1+O\left(z^{-2}\right) & i \frac{(b-a)}{4 z}+O\left(z^{-2}\right) \\
-i \frac{(b-a)}{4 z}+O\left(z^{-2}\right) & 1+O\left(z^{-2}\right)
\end{array}\right) \\
& =I+\frac{1}{z}\left(\begin{array}{cc}
0 & i \frac{(b-a)}{4 z} \\
-i \frac{(b-a)}{4 z} & 0
\end{array}\right)+O\left(z^{-2}\right) .
\end{aligned}
$$

\section{4}

\section{Parametrix at the edge points}

In the preceding section we obtained the asymptotic model RHP without considering the edge points. Indeed, at this points the jump matrix has a nasty behavior because it is obtained by intersection of four contours. To avoid this behavior, one can build a parametrix at this point. Consequently, we will 
Chapter 6. Large asymptotics of orthogonal polynomials: The Riemann-Hilbert approach

deform RHP in the neighborhood of this edge points, in such a way that inside this neighborhood, RHP can be solved exactly.

Consider small disks $D(a, r), D(b, r)$, such that

$$
D(a, r) \equiv\{z /|z-a| \leq r\}, D(b, r) \equiv\{z /|z-b| \leq r\}
$$

Next, for this moment we only consider the disk $D(b, r)$ at the edge point $b$. Outside this disk the solution $M$ is a good approximation to $S$. But inside this disk, $S$ is not a good solution of the jump conditions. To find a solution that solves the jump conditions which agrees with $M$ on the boundary of the disks $D$. We define a map from $D(b, r)$ to a neighborhood of 0 such that the jump contours are mapped to $\gamma^{ \pm} \cup \mathrm{R}$ where $\gamma^{ \pm}$correspond to rays of argument $\pm \theta$. For the purpose of defining this map, we proceed in the following way: First of all, from $\left(g_{-}+g_{+}=V+l\right)$ and the definition of $G$ we have

$$
\begin{aligned}
G(x) & =g_{+}(x)-\left(-g_{+}(x)+V(x)+l\right) \\
& =2 g_{+}(x)-V(x)-l,
\end{aligned}
$$

by (6.30), we get

$$
G(x)=2 g_{+}(x)-V(x)-l=\int_{x}^{b} h(y) \sqrt{(y-a)(y-b)}_{+} d y .
$$

Now, if we evaluate the integral at $x=b$, we obtain

$$
2 g_{+}(b)-V(b)-l=0,
$$

hence the constant $l$ is given by

$$
l=2 g_{+}(b)-V(b)
$$

replacing this $l$ in $G(x)$, one can write $G(x)$ as

$$
G(x)=2 g_{+}(x)-V(x)-\left(2 g_{+}(b)-V(b)\right)=\int_{x}^{b} h(y) \sqrt{(y-a)(y-b)} d y .
$$

The analytic extension of this equation to the upper half-plane is given by

$$
\left(2 g_{+}(b)-V(b)\right)-\left(2 g_{+}(z)-V(z)\right)=\int_{b}^{z} h(y) \sqrt{(y-a)(y-b)} d y,
$$


Chapter 6. Large asymptotics of orthogonal polynomials: The

since $f(y)=\sqrt{y-a} h(y)$ is analytic in a neighborhood of $y=b$, we can expand one factor of the integrand around $y=b$ in the following way

$$
\begin{aligned}
\left(2 g_{+}(b)-V(b)\right)-\left(2 g_{+}(z)-V(z)\right)= & \int_{b}^{z} \sqrt{(y-b)}(h(b) \sqrt{(b-a)}+ \\
& \left.+\sum_{j=1}^{\infty} f^{(j)}(b)(y-b)^{j}\right) d y,
\end{aligned}
$$

ordering and integrating, we have

$$
\begin{aligned}
\left(2 g_{+}(b)-V(b)\right)-\left(2 g_{+}(z)-V(z)\right)= & \sqrt{(b-a)} h(b) \int_{b}^{z} \sqrt{(y-b)}+ \\
& +\int_{b}^{z} \sum_{j=1}^{\infty} f^{(j)}(b)(y-b)^{j+\frac{1}{2}} d y \\
= & \sqrt{(b-a)} h(b) \frac{2}{3}(z-b)^{\frac{3}{2}}+ \\
& +\sum_{j=1}^{\infty} \frac{2 f^{(j)}(b)}{2 j+3}(z-b)^{j+\frac{3}{2}}
\end{aligned}
$$

if $z$ is sufficiently near $b$, we find

$$
\left(2 g_{+}(b)-V(b)\right)-\left(2 g_{+}(z)-V(z)\right)=\frac{2 \sqrt{(b-a)} h(b)}{3}(z-b)^{\frac{3}{2}}+O\left((z-b)^{\frac{5}{2}}\right),
$$

as function $\left(2 g_{+}(b)-V(b)\right)-\left(2 g_{+}(z)-V(z)\right)$ have jumps due to the factor $(z-b)^{\frac{3}{2}}$, this function can not define a conformal map. Now by the cancellation the exponent of the factor $(z-b)^{\frac{3}{2}}$ we can define a conformal map, in the following way

$$
\beta(z)=\left\{\frac{3}{4}\left[\left(2 g_{+}(b)-V(b)\right)-\left(2 g_{+}(z)-V(z)\right)\right]\right\}^{\frac{2}{3}}=\frac{(b-a)^{\frac{1}{3}} h^{\frac{2}{3}}(b)}{2^{\frac{2}{3}}}(z-b),
$$

where we only take the linear term due to proximity between $z$ and $b$.

Since we have the freedom to choose the image of $\beta(z)$ in the contours near $b$, matching the jump contours with the Stoke lines of the Airy function, we take $\theta=\frac{2 \pi}{3}$, hence the conformal map determines four regions in the disk $D(z, r)$ around 0 , such that $0<\arg \beta(z)<\frac{2 \pi}{3}$ defines region $\mathrm{I}, \frac{2 \pi}{3}<\arg \beta(z)<\pi$ defines region II and the other conjugates define regions III and IV respectively. Note that under the transformation,

$$
j_{Q}(z)=e^{-N\left(g_{-}(z)-\frac{V(z)}{2}-\frac{l}{2}\right) \sigma} j_{S}(z) e^{N\left(g_{+}(z)-\frac{V(z)}{2}-\frac{l}{2}\right) \sigma},
$$

the non constant jump matrices of $S$ convert to constant matrices in the neighborhood of $b$, in the following way 
Chapter 6. Large asymptotics of orthogonal polynomials: The

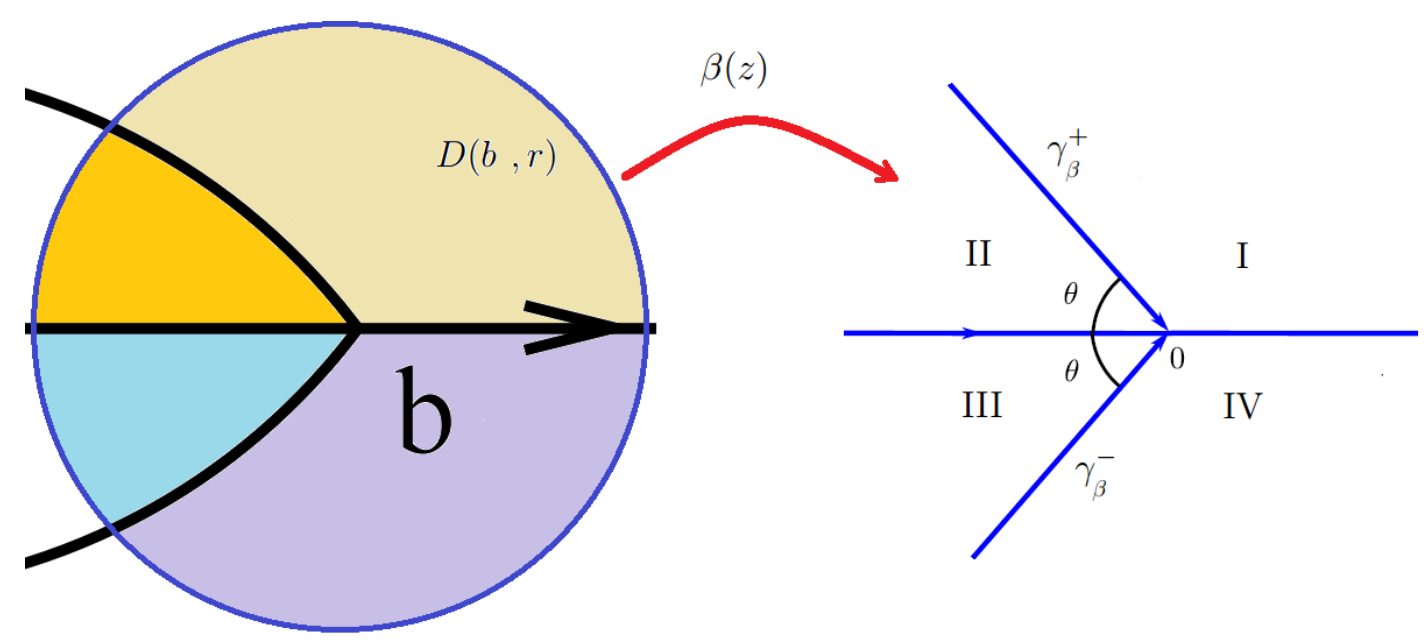

Figure 6.10: An illustration of the conformal map $\beta$.

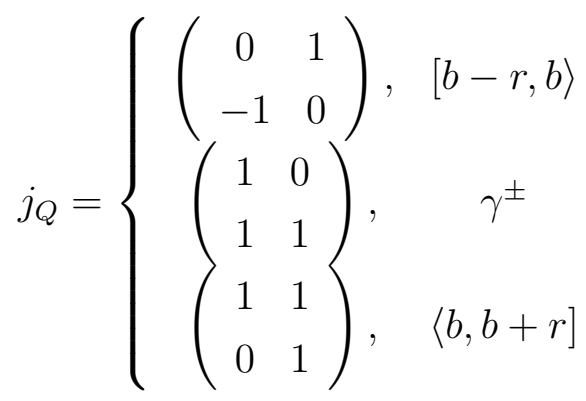

Then this jump matrices define the jump condition for some $Q_{N}$,

$$
\begin{aligned}
Q_{N+}(z) & =Q_{N-}(z) j_{Q}(z) \\
& =Q_{N-}(z) e^{-N\left(g_{-}(z)-\frac{V(z)}{2}-\frac{l}{2}\right) \sigma} j_{S}(z) e^{N\left(g_{+}(z)-\frac{V(z)}{2}-\frac{l}{2}\right) \sigma}
\end{aligned}
$$

multiplying both terms of this jump condition by $e^{-N\left(g_{+}(z)-\frac{V(z)}{2}-\frac{l}{2}\right) \sigma}$, we obtain

$$
\left(Q_{N+}(z) e^{-N\left(g_{+}(z)-\frac{V(z)}{2}-\frac{l}{2}\right) \sigma}\right)=\left(Q_{N-}(z) e^{-N\left(g_{-}(z)-\frac{V(z)}{2}-\frac{l}{2}\right) \sigma}\right) j_{S}(z)
$$

which is the jump condition for $S_{N}$ inside the disk $D(b, r)$. Thus the solution that solves the jump conditions of $S_{N}$ inside the disk $D(b, r)$ is given by

$$
U_{N}(z)=Q_{N}(z) e^{-N\left[g(z)-\frac{V(z)}{2}-\frac{l}{2}\right] \sigma_{3}}
$$

which is called the parametrix. Now to match this parametrix $U_{N}$ with $M$ on the boundary of the disk $D(b, r)$, we recur to a product of functions,

$$
Q_{N}(z)=E_{N}(z) \Phi\left(N^{\frac{2}{3}} \beta(z)\right)
$$

such that the first function allows to match $M$ and the other function reflects the jump conditions given by $j_{Q}$. Where we have used the conformal map and scaling to define the Airy parametrix, 
Chapter 6. Large asymptotics of orthogonal polynomials: The

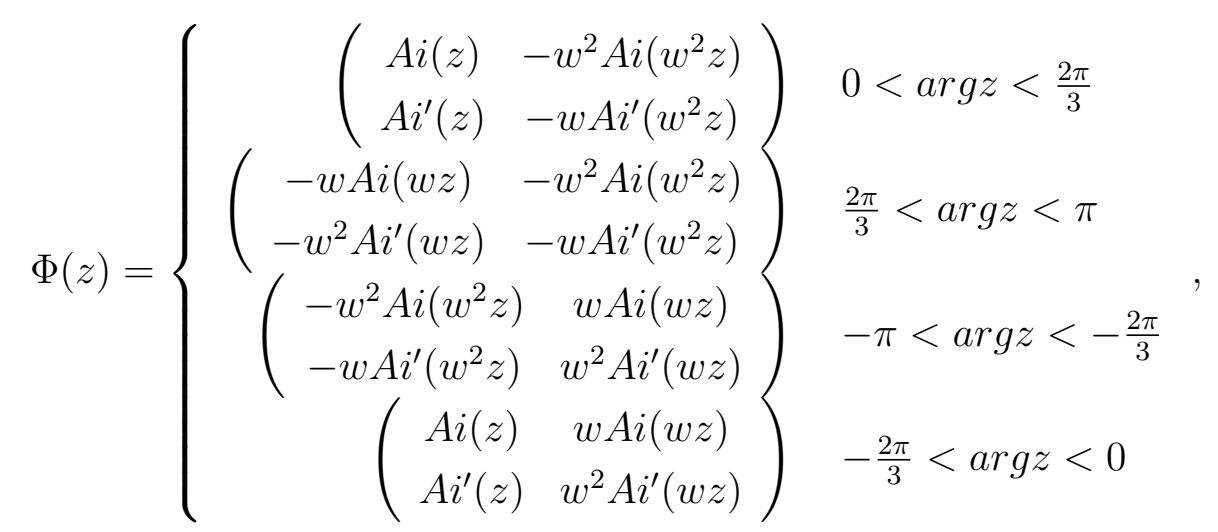

where $w=e^{\frac{2 \pi i}{3}}$. Recall that the Airy functions $A i(z), A i(w z), A i\left(w^{2} z\right)$ are solutions of the equation $y^{\prime \prime}=z y$, such that for $|\arg (z)|<\pi$ the Airy function has the following asymptotics at $z \rightarrow \infty$,

$$
\begin{aligned}
& A i(z)=\frac{1}{2 \sqrt{\pi} z^{\frac{1}{4}}} e^{-\frac{2}{3} z^{\frac{3}{2}}}\left(1+O\left(z^{-\frac{3}{2}}\right)\right) \\
& A i^{\prime}(z)=-\frac{z^{\frac{1}{4}}}{2 \sqrt{\pi}} e^{-\frac{2}{3} z^{\frac{3}{2}}}\left(1+O\left(z^{-\frac{3}{2}}\right)\right) .
\end{aligned}
$$

Furthermore, the Airy functions satisfy the following linear equations

$$
\begin{gathered}
A i(z)+w A i(w z)+w^{2} A i\left(w^{2} z\right)=0 \\
A i^{\prime}(z)+w^{2} A i^{\prime}(w z)+w A i^{\prime}\left(w^{2} z\right)=0 .
\end{gathered}
$$

Now we show that the Airy parametrix $\Phi(z)$ solves the jump conditions given by $j_{Q}$. then the jump of $\Phi(z)$ from the region IV to I, for $x \in\langle b, b+r]$ is given by

$$
\begin{aligned}
\Phi(x)_{I V}\left(\begin{array}{ll}
1 & 1 \\
0 & 1
\end{array}\right) & =\left(\begin{array}{cc}
A i(x) & w A i(w x) \\
A i^{\prime}(x) & w^{2} A i^{\prime}(w x)
\end{array}\right)\left(\begin{array}{ll}
1 & 1 \\
0 & 1
\end{array}\right) \\
& =\left(\begin{array}{cc}
A i(x) & A i(x)+w A i(w x) \\
A i^{\prime}(x) & A i^{\prime}(x)+w^{2} A i^{\prime}(w x)
\end{array}\right),
\end{aligned}
$$

by the linear relations $(6.84)(6.85)$, we find

$$
\begin{aligned}
& \Phi(x)_{I V}\left(\begin{array}{ll}
1 & 1 \\
0 & 1
\end{array}\right)=\left(\begin{array}{cc}
A i(x) & -w^{2} A i\left(w^{2} x\right) \\
A i^{\prime}(x) & -w A i^{\prime}\left(w^{2} x\right)
\end{array}\right) \\
& \Phi(x)_{I V}\left(\begin{array}{ll}
1 & 1 \\
0 & 1
\end{array}\right)=\Phi(x)_{I},
\end{aligned}
$$


Chapter 6. Large asymptotics of orthogonal polynomials: The

next, the jump of $\Phi(z)$ from the region II to I, for $x \in \gamma^{+}$is given by

$$
\begin{aligned}
\Phi(x)_{I I}\left(\begin{array}{ll}
1 & 0 \\
1 & 1
\end{array}\right) & =\left(\begin{array}{cc}
-w A i(w x) & -w^{2} A i\left(w^{2} x\right) \\
-w^{2} A i^{\prime}(w x) & -w A i^{\prime}\left(w^{2} x\right)
\end{array}\right)\left(\begin{array}{cc}
1 & 0 \\
1 & 1
\end{array}\right) \\
& =\left(\begin{array}{cc}
-w A i(w x)-w^{2} A i\left(w^{2} x\right) & -w^{2} A i\left(w^{2} x\right) \\
w^{2} A i^{\prime}(w x)-w A i^{\prime}\left(w^{2} x\right) & -w A i^{\prime}\left(w^{2} x\right)
\end{array}\right)
\end{aligned}
$$

by the linear relations $(6.84)(6.85)$, we get

$$
\begin{aligned}
& \Phi(x)_{I I}\left(\begin{array}{ll}
1 & 0 \\
1 & 1
\end{array}\right)=\left(\begin{array}{cc}
A i(x) & -w^{2} A i\left(w^{2} x\right) \\
A i^{\prime}(x) & -w A i^{\prime}\left(w^{2} x\right)
\end{array}\right) \\
& \Phi(x)_{I I}\left(\begin{array}{ll}
1 & 0 \\
1 & 1
\end{array}\right)=\Phi(x)_{I} .
\end{aligned}
$$

Similarly, for the other conjugates regions, we have

$$
\begin{aligned}
\Phi(x)_{I I I}\left(\begin{array}{cc}
0 & 1 \\
-1 & 0
\end{array}\right) & =\Phi(x)_{I I} \\
\Phi(x)_{I V}\left(\begin{array}{ll}
1 & 0 \\
1 & 1
\end{array}\right) & =\Phi(x)_{I I I} .
\end{aligned}
$$

Therefore, taking into account the orientation of the contours we deduce that

$$
\Phi_{+}(x)=\Phi_{-}(x) j_{Q}(x), \quad x \in \gamma^{ \pm} \cup \mathrm{R}
$$

Furthermore, the parametrix $U_{N}$ in terms of the Airy parametrix is given by

$$
U_{N}(z)=E_{N}(z) \Phi\left(N^{\frac{2}{3}} \beta(z)\right) e^{-N\left[g(z)-\frac{V(z)}{2}-\frac{l}{2}\right]},
$$

where the scaling $N^{\frac{2}{3}} \beta(z)$ is of order $O(1)$, because $\beta(z) \sim O\left(N^{\frac{3}{2}}\right)$. To match $M$ on the boundary of the disk $D(b, r), E_{N}$ can be written as a function of $M$. However, the asymptotic of the scaling Airy parametrix do not allow to do this exactly. Then, for hiding this asymptotic, we fit $E_{N}$ as

$$
E_{N}(z)=M(z) L_{N}^{-1}(z)
$$

where $L_{N}^{-1}(z)$ is the factor that matches $U_{N}$ with $M$, and is given by

$$
L_{N}(z)=\frac{1}{2 \sqrt{\pi}}\left(\begin{array}{cc}
N^{-\frac{1}{6}} \beta^{-\frac{1}{4}}(z) & 0 \\
0 & N^{\frac{1}{6}} \beta^{\frac{1}{4}}(z)
\end{array}\right)\left(\begin{array}{cc}
1 & i \\
-1 & i
\end{array}\right) .
$$


Chapter 6. Large asymptotics of orthogonal polynomials: The

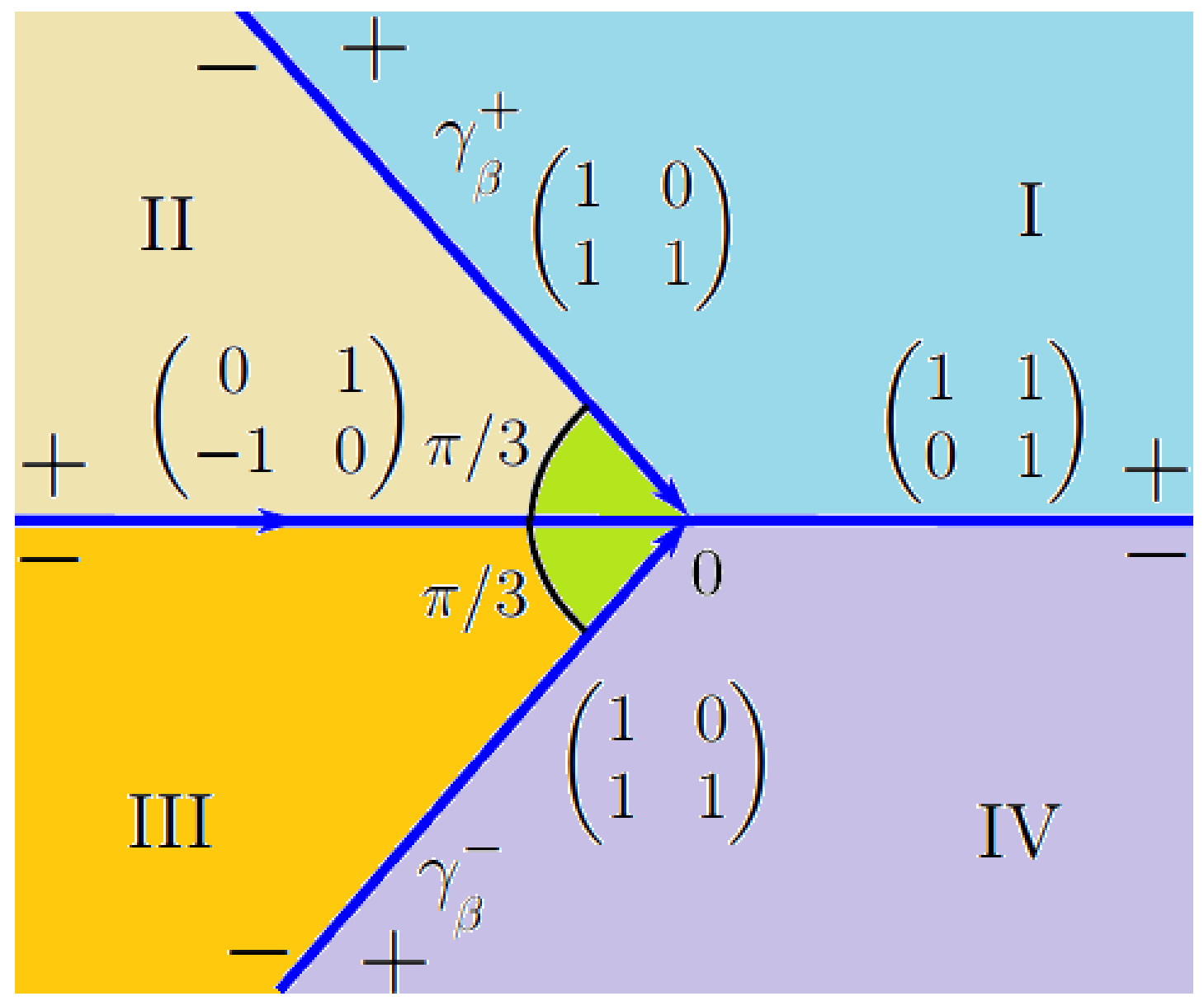

Figure 6.11: A representation of the Airy parametrix.

Notice that $L_{N}$ has at most a singularity of the fourth-root type at $b$ which is removable. In the same way, $M$ also has a removable singularity at $b$. Thence by a slight redefinition, both $L_{N}$ and $M$ are analytic in $D(b, r)$. Therefore, $E_{N}$ is analytic in $D(b, r)$. For the region I, by the asymptotic (6.82) of the Airy function we have

$$
-w^{2} A i\left(w^{2} z\right)=\frac{i}{2 \sqrt{\pi} z^{\frac{1}{4}}} e^{\frac{2}{3} z^{\frac{3}{2}}}\left(1+O\left(z^{-\frac{3}{2}}\right)\right),
$$

while, by the asymptotic (6.83) we obtain

$$
-w A i^{\prime}\left(w^{2} z\right)=\frac{i z^{\frac{1}{4}}}{2 \sqrt{\pi}} e^{\frac{2}{3} z^{\frac{3}{2}}}\left(1+O\left(z^{-\frac{3}{2}}\right)\right) .
$$

Therefore, the asymptotic behavior of the scaling Airy parametrix is given by

$$
\Phi\left(N^{\frac{2}{3}} \beta(z)\right)=
$$


Chapter 6. Large asymptotics of orthogonal polynomials: The

$$
\begin{aligned}
& \left(\begin{array}{cc}
\frac{e^{-\frac{2}{3}\left(N^{\frac{2}{3}} \beta(z)\right)^{\frac{3}{2}}}}{2 \sqrt{\pi}\left(N^{\frac{2}{3}} \beta(z)\right)^{\frac{1}{4}}}\left(1+O\left(\left(N^{\frac{2}{3}} \beta(z)\right)^{-\frac{3}{2}}\right)\right) & \frac{i e^{\frac{2}{3}\left(N^{\frac{2}{3}} \beta(z)\right)^{\frac{3}{2}}}}{2 \sqrt{\pi}\left(N^{\frac{2}{3}} \beta(z)\right)^{\frac{1}{4}}}\left(1+O\left(N^{-1} \beta(z)^{-\frac{3}{2}}\right)\right) \\
\frac{-\left(N^{\frac{2}{3}} \beta(z)\right)^{\frac{1}{4}}}{2 \sqrt{\pi} e^{\frac{2}{3}\left(N^{\frac{2}{3}} \beta(z)\right)^{\frac{3}{2}}}\left(1+O\left(\left(N^{\frac{2}{3}} \beta(z)\right)^{-\frac{3}{2}}\right)\right)} & \frac{i\left(N^{\frac{2}{3}} \beta(z)\right)^{\frac{1}{4}}}{2 \sqrt{\pi} e^{-\frac{2}{3}\left(N^{\frac{2}{3}} \beta(z)\right)^{\frac{3}{2}}}}\left(1+O\left(N^{-1} \beta(z)^{-\frac{3}{2}}\right)\right)
\end{array}\right) \\
& \Phi\left(N^{\frac{2}{3}} \beta(z)\right)=\left(\begin{array}{cc}
\frac{1}{2 \sqrt{\pi} N^{\frac{1}{6}} \beta^{\frac{1}{4}}} e^{-\frac{2}{3} N \beta^{\frac{3}{2}}} & \frac{i}{2 \sqrt{\pi} N^{\frac{1}{6}} \beta^{\frac{1}{4}}} e^{\frac{2}{3} N \beta^{\frac{3}{2}}} \\
\frac{-N^{\frac{1}{6}} \beta^{\frac{1}{4}}}{2 \sqrt{\pi}} e^{-\frac{2}{3} N \beta^{\frac{3}{2}}} & \frac{i N^{\frac{1}{6}} \beta^{\frac{1}{4}}}{2 \sqrt{\pi}} e^{\frac{2}{3} N \beta^{\frac{3}{2}}}
\end{array}\right)\left(I+O\left(N^{-1}\right)\right) \\
& =\frac{1}{2 \sqrt{\pi}}\left(\begin{array}{cc}
\frac{1}{N^{\frac{1}{6}} \beta^{\frac{1}{4}}} e^{-\frac{2}{3} N \beta^{\frac{3}{2}}} & \frac{i}{N^{\frac{1}{6}} \beta^{\frac{1}{4}}} e^{\frac{2}{3} N \beta^{\frac{3}{2}}} \\
-N^{\frac{1}{6}} \beta^{\frac{1}{4}} e^{-\frac{2}{3} N \beta^{\frac{3}{2}}} & i N^{\frac{1}{6}} \beta^{\frac{1}{4}} e^{\frac{2}{3} N \beta^{\frac{3}{2}}}
\end{array}\right)\left(I+O\left(N^{-1}\right)\right) \\
& =\frac{1}{2 \sqrt{\pi}}\left(\begin{array}{cc}
N^{-\frac{1}{6}} \beta^{-\frac{1}{4}} & 0 \\
0 & N^{\frac{1}{6}} \beta^{\frac{1}{4}}
\end{array}\right)\left(\begin{array}{cc}
e^{-\frac{2}{3} N \beta^{\frac{3}{2}}} & i e^{\frac{2}{3} N \beta^{\frac{3}{2}}} \\
-e^{-\frac{2}{3} N \beta^{\frac{3}{2}}} & i e^{\frac{2}{3} N \beta^{\frac{3}{2}}}
\end{array}\right) \times \\
& \times\left(I+O\left(N^{-1}\right)\right) \\
& =\frac{1}{2 \sqrt{\pi}}\left(\begin{array}{cc}
N^{-\frac{1}{6}} \beta^{-\frac{1}{4}} & 0 \\
0 & N^{\frac{1}{6}} \beta^{\frac{1}{4}}
\end{array}\right)\left(\begin{array}{cc}
1 & i \\
-1 & i
\end{array}\right) \times \\
& \times\left(\begin{array}{cc}
e^{-\frac{2}{3} N \beta^{\frac{3}{2}}} & 0 \\
0 & e^{\frac{2}{3} N \beta^{\frac{3}{2}}}
\end{array}\right)\left(I+O\left(N^{-1}\right)\right) \\
& \Phi\left(N^{\frac{2}{3}} \beta(z)\right)=\frac{1}{2 \sqrt{\pi}} N^{-\frac{1}{6} \sigma_{3}} \beta(z)^{-\frac{1}{4} \sigma_{3}}\left(\begin{array}{cc}
1 & i \\
-1 & i
\end{array}\right)\left(I+O\left(N^{-1}\right)\right) e^{-\frac{2}{3} N \beta(z)^{\frac{3}{2}} \sigma_{3}},
\end{aligned}
$$

by definition of the conformal map, $\frac{2}{3} \beta(z)^{\frac{3}{2}}=-g(z)+\frac{V(z)}{2}+\frac{l}{2}$, we have

$$
\Phi\left(N^{\frac{2}{3}} \beta(z)\right)=\frac{N^{-\frac{1}{6} \sigma_{3}}}{2 \sqrt{\pi}} \beta(z)^{-\frac{1}{4} \sigma_{3}}\left(\begin{array}{cc}
1 & i \\
-1 & i
\end{array}\right)\left(I+O\left(N^{-1}\right)\right) e^{\left(N g(z)-\frac{N V(z)}{2}-\frac{N l}{2}\right) \sigma_{3}} .
$$

Then, using this expression in the parametrix $U_{N}$, we get

$$
\begin{aligned}
U_{N}(z)= & E_{N}(z) \frac{1}{2 \sqrt{\pi}} N^{-\frac{1}{6} \sigma_{3}} \beta(z)^{-\frac{1}{4} \sigma_{3}}\left(\begin{array}{cc}
1 & i \\
-1 & i
\end{array}\right)\left(I+O\left(N^{-1}\right)\right) \times \\
& \times e^{\left(N g(z)-\frac{N V(z)}{2}-\frac{N l}{2}\right) \sigma_{3}} e^{-N\left[g(z)-\frac{V(z)}{2}-\frac{l}{2}\right] \sigma_{3}} \\
= & E_{N}(z) \frac{1}{2 \sqrt{\pi}} N^{-\frac{1}{6} \sigma_{3}} \beta(z)^{-\frac{1}{4} \sigma_{3}}\left(\begin{array}{cc}
1 & i \\
-1 & i
\end{array}\right)\left(I+O\left(N^{-1}\right)\right),
\end{aligned}
$$

according to the fitted $E_{N}$ we find

$$
U_{N}(z)=M(z) L_{N}^{-1}(z) \frac{1}{2 \sqrt{\pi}} N^{-\frac{1}{6} \sigma_{3}} \beta(z)^{-\frac{1}{4} \sigma_{3}}\left(\begin{array}{cc}
1 & i \\
-1 & i
\end{array}\right)\left(I+O\left(N^{-1}\right)\right)
$$


Chapter 6. Large asymptotics of orthogonal polynomials: The

notice that $\frac{1}{2 \sqrt{\pi}} N^{-\frac{1}{6} \sigma_{3}} \beta(z)^{-\frac{1}{4} \sigma_{3}}\left(\begin{array}{cc}1 & i \\ -1 & i\end{array}\right)=L_{N}(z)$, then we have

$$
\begin{aligned}
U_{N}(z) & =M(z) L_{N}^{-1}(z) L_{N}(z)\left(I+O\left(N^{-1}\right)\right) \\
U_{N}(z) & =M(z)\left(I+O\left(N^{-1}\right)\right) .
\end{aligned}
$$

For the region II, by the asymptotics (6.82) (6.83) of the Airy function we have

$$
\begin{aligned}
-w A i(w z) & =\frac{1}{2 \sqrt{\pi} z^{\frac{1}{4}}} e^{-\frac{2}{3} z^{\frac{3}{2}}}\left(1+O\left(z^{-\frac{3}{2}}\right)\right) \\
-w^{2} A i^{\prime}(w z) & =\frac{-z^{\frac{1}{4}}}{2 \sqrt{\pi}} e^{-\frac{2}{3} z^{\frac{3}{2}}}\left(1+O\left(z^{-\frac{3}{2}}\right)\right) .
\end{aligned}
$$

Thus, the scaling Airy parametrix has the following asymptotic behavior

$$
\Phi\left(N^{\frac{2}{3}} \beta(z)\right)=\frac{1}{2 \sqrt{\pi}} N^{-\frac{1}{6} \sigma_{3}} \beta(z)^{-\frac{1}{4} \sigma_{3}}\left(\begin{array}{cc}
1 & i \\
-1 & i
\end{array}\right)\left(I+O\left(N^{-1}\right)\right) e^{-\frac{2}{3} N \beta(z)^{\frac{3}{2}} \sigma_{3}},
$$

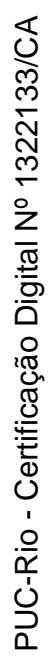

replacing the conformal map, $\frac{2}{3} \beta(z)^{\frac{3}{2}}=-g(z)+\frac{V(z)}{2}+\frac{l}{2}$, this asymptotic behavior is given by

$$
\Phi\left(N^{\frac{2}{3}} \beta(z)\right)=\frac{N^{-\frac{1}{6} \sigma_{3}}}{2 \sqrt{\pi}} \beta(z)^{-\frac{1}{4} \sigma_{3}}\left(\begin{array}{cc}
1 & i \\
-1 & i
\end{array}\right)\left(I+O\left(N^{-1}\right)\right) e^{\left(N g(z)-\frac{N V(z)}{2}-\frac{N l}{2}\right) \sigma_{3}} .
$$

Therefore, putting this asymptotic behavior in the parametrix $U_{N}$, we find

$$
\begin{aligned}
U_{N}(z)= & E_{N}(z) \frac{1}{2 \sqrt{\pi}} N^{-\frac{1}{6} \sigma_{3}} \beta(z)^{-\frac{1}{4} \sigma_{3}}\left(\begin{array}{cc}
1 & i \\
-1 & i
\end{array}\right)\left(I+O\left(N^{-1}\right)\right) \times \\
& \times e^{\left(N g(z)-\frac{N V(z)}{2}-\frac{N l}{2}\right) \sigma_{3}} e^{-N\left[g(z)-\frac{V(z)}{2}-\frac{l}{2}\right] \sigma_{3}} \\
= & E_{N}(z) \frac{1}{2 \sqrt{\pi}} N^{-\frac{1}{6} \sigma_{3}} \beta(z)^{-\frac{1}{4} \sigma_{3}}\left(\begin{array}{cc}
1 & i \\
-1 & i
\end{array}\right)\left(I+O\left(N^{-1}\right)\right),
\end{aligned}
$$

by the definition of $E_{N}$ we have

$$
U_{N}(z)=M(z) L_{N}^{-1}(z) \frac{1}{2 \sqrt{\pi}} N^{-\frac{1}{6} \sigma_{3}} \beta(z)^{-\frac{1}{4} \sigma_{3}}\left(\begin{array}{cc}
1 & i \\
-1 & i
\end{array}\right)\left(I+O\left(N^{-1}\right)\right)
$$

Since, $\frac{1}{2 \sqrt{\pi}} N^{-\frac{1}{6} \sigma_{3}} \beta(z)^{-\frac{1}{4} \sigma_{3}}\left(\begin{array}{cc}1 & i \\ -1 & i\end{array}\right)=L_{N}(z)$, then we obtain

$$
\begin{aligned}
& U_{N}(z)=M(z) L_{N}^{-1}(z) L_{N}(z)\left(I+O\left(N^{-1}\right)\right) \\
& U_{N}(z)=M(z)\left(I+O\left(N^{-1}\right)\right) .
\end{aligned}
$$


Chapter 6. Large asymptotics of orthogonal polynomials: The Riemann-Hilbert approach

Similarly to the other conjugates regions the parametrix $U_{N}$ match with $M$ on the boundary of the disk $D(b, r)$.

\section{5}

\section{Final transformation and solution of the RHP}

In this section we glue the model RHP to the parametrix, resulting one RHP for $R_{N}$ in all the complex plane C. The solution of this RHP can be obtained

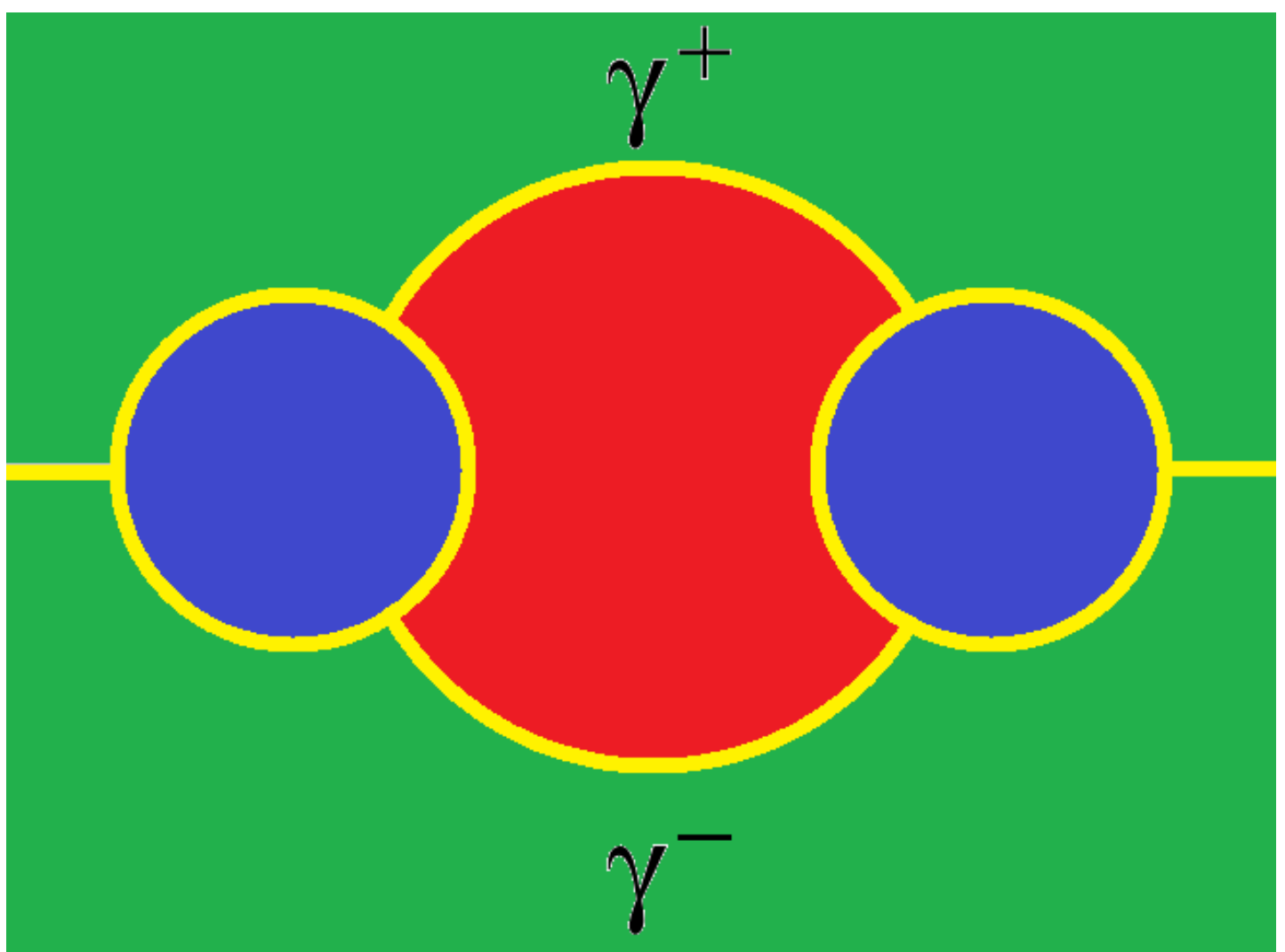

Figure 6.12: Analytic continuation of the jump condition after glueing the model RHP to the parametrix.

by perturbation series and is close to $I$.

Let $R_{N}$ solves the final RHP $\left(\gamma, j_{R}\right)$ :

1. $R_{N}(z): \mathrm{C} \backslash \gamma \rightarrow \mathrm{C}^{2 \times 2}$ is analytic, such that

$$
\gamma=\gamma^{+} \cup \gamma^{-} \cup \partial D(a, r) \cup \partial D(a, r) \cup \mathrm{R} \backslash[a-r, b+r]
$$

oriented as in Figure 6.12.

2. For $z \in \gamma$,

$$
R_{N+}(z)=R_{N-} j_{R}(z)
$$


Chapter 6. Large asymptotics of orthogonal polynomials: The Riemann-Hilbert approach

where

$$
j_{R}(z)=\left\{\begin{array}{cc}
M(z) U_{N}^{-1}(z) & , \partial D(a, r) \cup \partial D(a, r) \\
M(z) j_{S}(z) M^{-1}(z) & , \gamma^{ \pm} \cup \mathrm{R} \backslash[a-r, b+r]
\end{array}\right.
$$

3. As $z \rightarrow \infty$,

$$
R_{N}(z) \cong I+\sum_{j=1}^{\infty} \frac{R_{j}}{z^{j}}
$$

where for $z \in \partial D(a, r) \cup \partial D(a, r)$

$$
\begin{aligned}
j_{R}(z) & \cong M(z)\left(M(z)\left(I+O\left(N^{-1}\right)\right)\right)^{-1} \\
& \cong I+O\left(N^{-1}\right)
\end{aligned}
$$

and for $z \in \gamma^{ \pm} \cup \mathrm{R} \backslash[a-r, b+r]$

$$
j_{R}(z) \cong\left\{\begin{array}{cc}
\left(\begin{array}{cc}
1 & 0 \\
O\left(e^{-c N}\right) & 1
\end{array}\right) M(z) M^{-1}(z), & \gamma^{ \pm} \\
1 & O\left(e^{-c N}\right) \\
0 & 1
\end{array}\right) M(z) M^{-1}(z), \quad \mathrm{R} \backslash[a-r, b+r]
$$

Therefore

$$
j_{R}(z) \cong I+O\left(e^{-c N}\right) .
$$

Now If we represent the RHP $\left(\gamma, j_{R}\right)$ as singular integral equation, we obtain

$$
R_{N-}(z)-I=\frac{1}{2 \pi i} \int_{\gamma} \frac{R_{N-}\left(z^{\prime}\right)\left(j_{R}\left(z^{\prime}\right)-I\right)}{z^{\prime}-(z-i \epsilon)} d z^{\prime} .
$$

Since

$$
j_{R}\left(z^{\prime}\right)-I \cong \begin{cases}O\left(N^{-1}\right) & , \partial D(a, r) \cup \partial D(a, r) \\ O\left(e^{-c N}\right) & , \gamma^{ \pm} \cup \mathrm{R} \backslash[a-r, b+r]\end{cases}
$$

The singular integral equation can be solved by perturbation series, and by the normalization condition of the RHP, we have

$$
R_{N-}(z)=\sum_{k=0}^{\infty} R_{N-, k}(z)
$$

such that $R_{N-, 0}=I$, then the singular integral equation can be written as follows

$$
\sum_{k=0}^{\infty} R_{N-, k}(z)-I=\frac{1}{2 \pi i} \int_{\gamma} \frac{\sum_{k=0}^{\infty} R_{N-, k}\left(z^{\prime}\right)\left(j_{R}\left(z^{\prime}\right)-I\right)}{z^{\prime}-(z-i \epsilon)} d z^{\prime}
$$


Chapter 6. Large asymptotics of orthogonal polynomials: The Riemann-Hilbert approach

hence

$$
\begin{aligned}
\sum_{k=1}^{\infty} R_{N-, k}(z) & =\frac{1}{2 \pi i} \int_{\gamma} \frac{\sum_{k=0}^{\infty} R_{N-, k}\left(z^{\prime}\right)\left(j_{R}\left(z^{\prime}\right)-I\right)}{z^{\prime}-(z-i \epsilon)} d z^{\prime} \\
& =\sum_{k=0}^{\infty} \frac{1}{2 \pi i} \int_{\gamma} \frac{R_{N-, k}\left(z^{\prime}\right)\left(j_{R}\left(z^{\prime}\right)-I\right)}{z^{\prime}-(z-i \epsilon)} d z^{\prime}
\end{aligned}
$$

by the properties of the indices in the sum,

$$
\sum_{k=1}^{\infty} R_{N-, k}(z)=\sum_{k=1}^{\infty} \frac{1}{2 \pi i} \int_{\gamma} \frac{R_{N-, k-1}\left(z^{\prime}\right)\left(j_{R}\left(z^{\prime}\right)-I\right)}{z^{\prime}-(z-i \epsilon)} d z^{\prime}
$$

thus,

$$
R_{N-, k}(z)=\frac{1}{2 \pi i} \int_{\gamma} \frac{R_{N-, k-1}\left(z^{\prime}\right)\left(j_{R}\left(z^{\prime}\right)-I\right)}{z^{\prime}-(z-i \epsilon)} d z^{\prime} .
$$

As $R_{N-, 0}=I$, we find that

$$
R_{N-, 1}(z)=\frac{1}{2 \pi i} \int_{\gamma} \frac{\left(j_{R}\left(z^{\prime}\right)-I\right)}{z^{\prime}-(z-i \epsilon)} d z^{\prime}
$$

without loss of generality, as $j_{R}\left(z^{\prime}\right)-I \cong O\left(N^{-1}\right)$ we have

$$
R_{N-, 1}(z) \cong O\left(N^{-1}\right)
$$

and for the next term

$$
\begin{aligned}
R_{N-, 2}(z) & =\frac{1}{2 \pi i} \int_{\gamma} \frac{R_{N-, 1}\left(z^{\prime}\right) O\left(N^{-1}\right)}{z^{\prime}-(z-i \epsilon)} d z^{\prime} \\
& =\frac{1}{2 \pi i} \int_{\gamma} \frac{O\left(N^{-1}\right) O\left(N^{-1}\right)}{z^{\prime}-(z-i \epsilon)} d z^{\prime} \\
R_{N-, 2}(z) & \cong O\left(N^{-2}\right) .
\end{aligned}
$$

Consequently,

$$
R_{N-, k}(z) \cong O\left(N^{-k}\right) .
$$

Therefore, the solution of the singular integral equation is given by

$$
\begin{aligned}
R_{N-}(z) & \cong I+\sum_{k=1}^{\infty} O\left(N^{-k}\right) \\
& \cong I+O\left(N^{-1}\right)
\end{aligned}
$$


Chapter 6. Large asymptotics of orthogonal polynomials: The Riemann-Hilbert approach

By the jump condition,

$$
\begin{aligned}
R_{N+}(z) & =R_{N-}(z) j_{R}(z) \\
& \cong\left(I+O\left(N^{-1}\right)\right) j_{R}(z),
\end{aligned}
$$

as $j_{R}(z)=I+O\left(N^{-1}\right)$

$$
\begin{aligned}
& R_{N+}(z) \cong\left(I+O\left(N^{-1}\right)\right)\left(I+O\left(N^{-1}\right)\right) \\
& R_{N+}(z)=I+O\left(N^{-1}\right) .
\end{aligned}
$$

Therefore by Liouville's theorem we obtain

$$
R_{N}(z)=I+O\left(N^{-1}\right)
$$

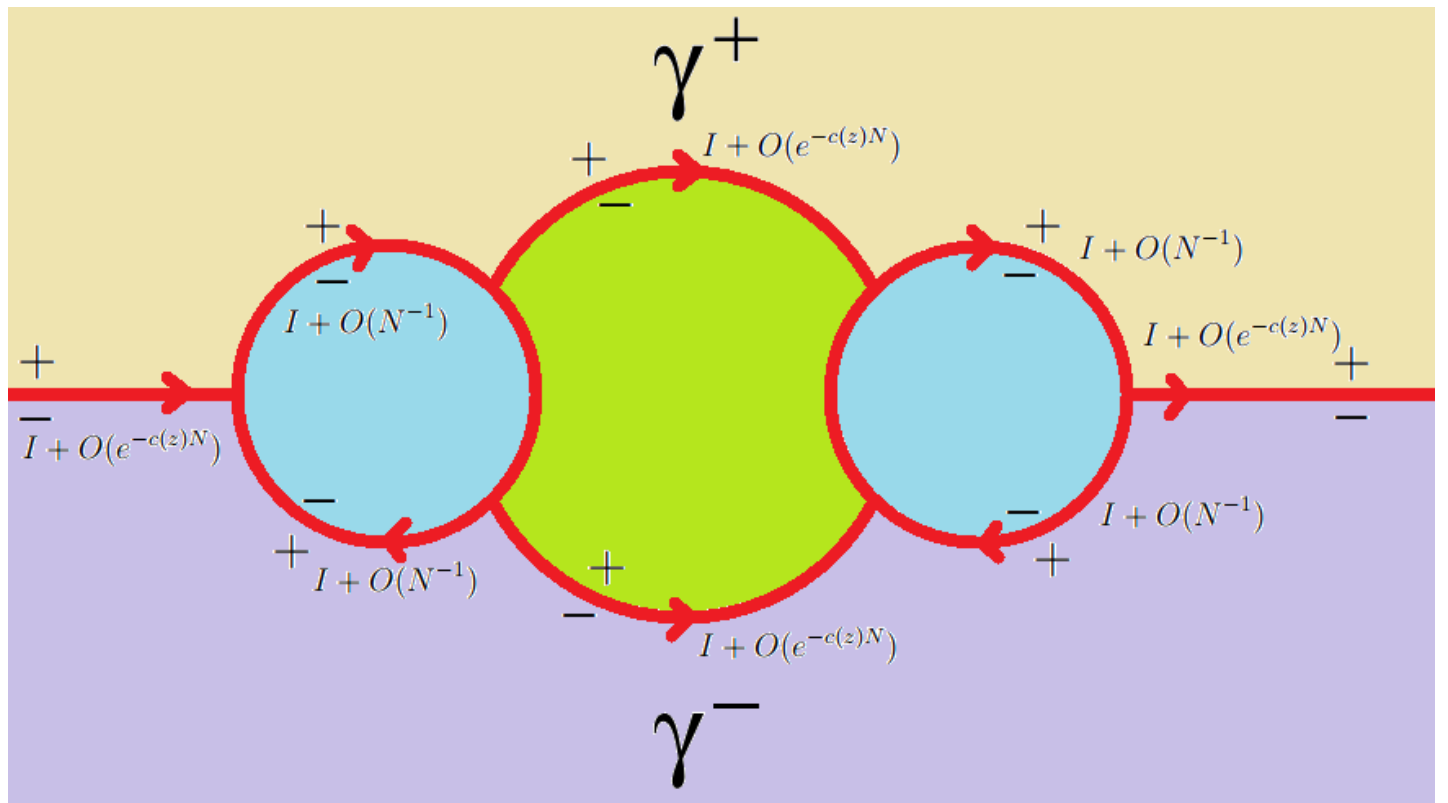

Figure 6.13: After the final transformation $R_{N}$ both jump and asymptotic conditions of the RHP approach to the identity when $N \rightarrow \infty$.

\section{6}

\section{Large asymptotics of orthogonal polynomials}

In the RMT we are interesed in calculating limit density, limit local distribution and free energy, that is closely related to the large asymptotic of orthogonal 
Chapter 6. Large asymptotics of orthogonal polynomials: The Riemann-Hilbert approach

polynomials. Now we can go back to the tranformations

$$
\begin{aligned}
R_{N}(z)= & I+O\left(N^{-1}\right) \\
S_{N}(z)= & \left\{\begin{array}{c}
R_{N}(z) M(z),, z \in \mathrm{C} \backslash D(a, r) \cup(b, r) \\
R_{N}(z) U_{N}(z), z \in D(a, r) \cup(b, r)
\end{array}\right. \\
T_{N}(z)= & \left\{\begin{array}{c}
S_{N}(z)\left(\begin{array}{cc}
1 & 0 \\
e^{-N G(z)} & 1
\end{array}\right) \\
S_{N}(z)\left(\begin{array}{cc}
1 & 0 \\
e^{N G(z)} & 1
\end{array}\right)^{-1}, z \in \mathcal{L}^{+} \\
S_{N}(z)
\end{array}, z \in \mathcal{L}^{-}\right. \\
Y_{N}(z)= & e^{\frac{N l}{2} \sigma_{3}} T_{N}(z) e^{N\left[g(z)-\frac{l}{2}\right] \sigma_{3}}
\end{aligned}
$$
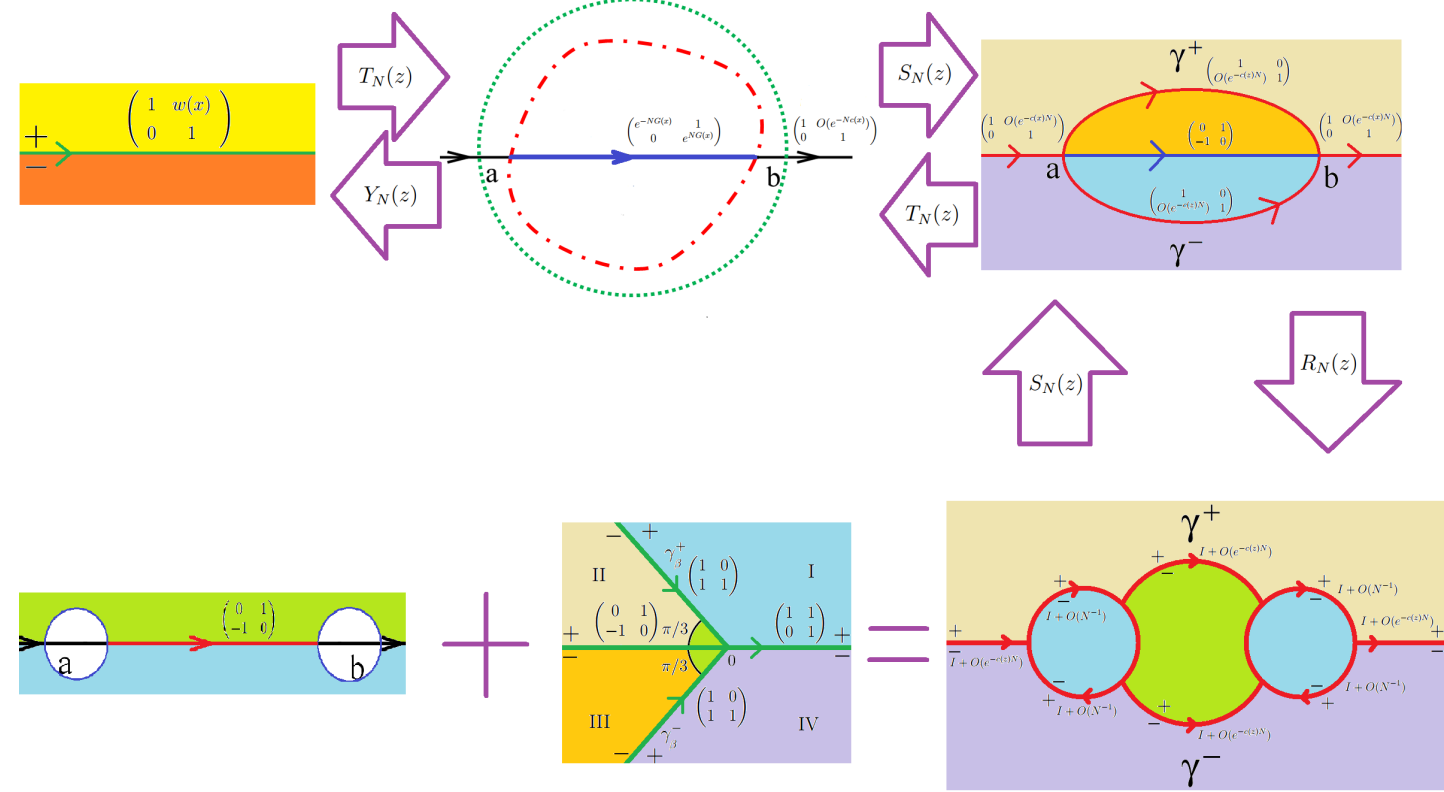

Figure 6.14: Screenplay of the steepest descent method for solving the RHP that containing information about orthogonal polynomials.

\section{7}

\section{Concluding remarks}

In this chapter we studied the steepest descent method in the RHP which consists in a serie of transformations for approaching the jump and asymptotic conditions of the RHP to the identity. Recently large asymptotics of orthogonal polynomials have attracted a lot of attention because of their relation to the statistics of the eigenvalues of Hermitian random $N \times N$ matrix, as $N \rightarrow \infty$. Importantly, this statistics have seen to be dependent only on symmetry properties of the matrices under consideration, but not on the exact distribution of 
Chapter 6. Large asymptotics of orthogonal polynomials: The Riemann-Hilbert approach

the entries of the matrix. This behavior is known as universality. Most interestingly, the local statistics at the edge has a universality independent of the potential $V(x)$. The bulk scaling limit as $N \rightarrow \infty$ is described by [17]

$$
\lim _{N \rightarrow \infty} \frac{1}{N c} K_{N}\left(z+\frac{u}{N c}, z+\frac{v}{N c}\right)=\frac{\sin (\pi(u-v))}{\pi(u-v)} .
$$

and for the edge point $b$ is given by [17]

$$
\lim _{N \rightarrow \infty} \frac{1}{(N c)^{\frac{2}{3}}} K_{N}\left(b+\frac{u}{(N c)^{\frac{2}{3}}}, b+\frac{v}{(N c)^{\frac{2}{3}}}\right)=\frac{A i(u) A i^{\prime}(v)-A i^{\prime}(u) A i(v)}{u-v} .
$$

Simirlarly for the other edge point $a$,

$$
\lim _{N \rightarrow \infty} \frac{1}{(N c)^{\frac{2}{3}}} K_{N}\left(a-\frac{u}{(N c)^{\frac{2}{3}}}, a-\frac{v}{(N c)^{\frac{2}{3}}}\right)=\frac{A i(u) A i^{\prime}(v)-A i^{\prime}(u) A i(v)}{u-v},
$$

where the rescaling is the same for both edge points. 


\section{7}

\section{Discussion and Outlook}

In this thesis we studied the theory necessary to formalize the continuum limit of Random Matrix Theory via Riemann Hilbert problems. Especifically we have seen the planar limit which is the minimal and standard continuum theory that one can find in almost all the applications of Random Matrix Theory. To get the other continuum theories by Riemann Hilbert theory one only has to change the local parametrix and the rest of transformations keeping in the same way. For this reason the Riemann Hilbert algorithm can be extended to other continuum theories without a lot of difficulties. For example in the Dynamical Triangulation continuum theory the parametrix local is given by the painleve I which is a nonlinear function that also can be analyzed by Riemann Hilbert problems. Indeed with the techniques of the Riemann Hilbert problems one can demostrate the transition to continuum theories and study the continuum theory by steepest descent method. Other interesting subject that was studied in this thesis is the variety of representations of the orthogonal polynomials. Motivating to continue to look more representations of the orthogonal polynomials. As the generating function for rooted branched polymers is given by generating function of the Dick paths, we also have a manifold of representations of the Gaussian Unitary Ensemble because one can think of the Hermite polynomials as a characterization of this ensemble without rigorosity and in this way connect this ensemble to the variety of representations of the orthogonal polynomials. We remarked the Gaussian Unitary Ensemble because this corresponds to the same family of continuum theories of the planar limit. So incrementing the quantity of tools for analysing this family of continuum theories that range from Riemann Hilbert problems to Gaussian Unitary Ensemble. Other ensembles as Circular Ensemble also correspond to this family of continuum theories allowing to find other applications as in the number theory. On the other side the Dyson gas allows one to interpret the statistical information of the eigenvalues of these ensembles. All these connections can be applied to the nonperturbative gravitational path integral of Dynamical Triangulation and can be extended to its causal approach in the discrete and continuum side because both correspond to Random Matrix Theories. 


\section{A \\ Continum limits of Random matrices}

Before describing the continuum limit of the matrix integral, we review how the phase transition of the path integral gives a quantum continuum theory with an error proportional to the inverse of the number $N$ of random paths. This transition occurs after decomposing the time interval into $N$ small intervals when we take the large $N$ limit. Where the propagator is the sum of all possible paths with lattice spacing $a$ from some field configuration to other field configuration such that to each path we associate a phase.

$$
\left\langle\psi_{f} \mid \psi_{i}\right\rangle=\int[d \psi] e^{i S(\psi)}
$$

By the transformation $t=-i \tau$ that changes the Minkowski into Euclidean space time, the propagator in the evolution kernel is analytically continued from the imaginary time to real time and hence into Euclidean time propagator,

$$
\left\langle\psi_{f} \mid \psi_{i}\right\rangle_{E}=\int[d \psi]_{E} e^{-S_{E}(\psi)}
$$

which is given by a partition function at finite temperature with a Gibbs measure, where paths have the only constraint that they are periodic in Euclidean time with period $\tau=\beta$.

In both propagators as Minkowskian and Euclidean, the integral is well defined by taking the spacing $a$ to zero and at the same time taking the number $N$ of intervals to infinite, hence we obtain a right quantum theory by a proper scaling limit.

In the same way that the Euclidean propagator, the matrix integrals correspond to path integrals where the paths are discrete graphs such that a planar limit is done by taking the area $A$ of this graphs to zero and at the same time taking the number $N$ of graphs to infinite. In this way the matrix integrals acquire meaning as a continuum theory with free energy

$$
F_{N}^{0}=\sum_{\text {connected } \Gamma} N^{2-2 g} F^{g}
$$


which is the same for a family of random graphs. Since this family after taking the planar limit are well defined into a finite radius of convergence $g_{c}$, so when the coupling constant reaches this radius of convergence $g_{c}$, the free energy is dominated by graphs with infinite vertices which corresponds to a continuum surface. In other words this limit only combines graphs of the same genus $g=0$.

If one wants the contribution of all the genus in the free energy and as the divergence of the terms of this energy is a local phenomena and not dependt on global properties likewise the genus, we take the limits $N \rightarrow \infty$ and $g \rightarrow g_{c}$ in a correlated manner such that genus suppression is compensated with $g \rightarrow g_{c}$. This so-called double scaling limit gives a coherent contribution from all genus in the free energy. Moreover this energy corresponds to a family of continuum theories differents from the families obtained by a planar limit. 


\section{B}

\section{Properties of the kernel}

As the determinantal structure of the point process on the Hermitian case is described by the kernel, so the kernel $K_{N}\left(\lambda_{i}, \lambda_{j}\right)=$ $e^{-\frac{1}{2} N\left(V\left(\lambda_{i}\right)+V\left(\lambda_{j}\right)\right)} \sum_{n=0}^{N-1} \frac{1}{h_{n}} p_{n}\left(\lambda_{i}\right) p_{n}\left(\lambda_{j}\right)$, fullfiled the following property

$$
\begin{aligned}
\int K_{N}\left(\lambda_{i}, \lambda_{k}\right) K_{N}\left(\lambda_{k}, \lambda_{j}\right) d \lambda_{k}= & \int d \lambda_{k} e^{-\frac{1}{2} N\left(V\left(\lambda_{i}\right)+2 V\left(\lambda_{k}\right)+V\left(\lambda_{j}\right)\right)} \sum_{n=0}^{N-1} p_{n}\left(\lambda_{i}\right) p_{n}\left(\lambda_{k}\right) \\
& \times \sum_{m=0}^{N-1} p_{m}\left(\lambda_{k}\right) p_{m}\left(\lambda_{j}\right) \\
= & \sum_{n, m=0}^{N-1} \int d \lambda_{k} e^{-N V\left(\lambda_{k}\right)} e^{-\frac{1}{2} N\left(V\left(\lambda_{i}\right)+V\left(\lambda_{j}\right)\right)} p_{n}\left(\lambda_{i}\right) \times \\
& \times p_{n}\left(\lambda_{k}\right) p_{m}\left(\lambda_{k}\right) p_{m}\left(\lambda_{j}\right) \\
= & \sum_{n, m=0}^{N-1} e^{-\frac{1}{2} N\left(V\left(\lambda_{i}\right)+V\left(\lambda_{j}\right)\right)} p_{n}\left(\lambda_{i}\right) \delta_{n, m} p_{m}\left(\lambda_{j}\right) \\
= & \sum_{n=0}^{N-1} e^{-\frac{1}{2} N\left(V\left(\lambda_{i}\right)+V\left(\lambda_{j}\right)\right)} p_{n}\left(\lambda_{i}\right) p_{n}\left(\lambda_{j}\right) \\
\int K_{N}\left(\lambda_{i}, \lambda_{k}\right) K_{N}\left(\lambda_{k}, \lambda_{j}\right) d \lambda_{k}= & K_{N}\left(\lambda_{i}, \lambda_{j}\right),
\end{aligned}
$$

and has the next property

$$
\begin{aligned}
\int K_{N}\left(\lambda_{i}, \lambda_{i}\right) d \lambda_{i} & =\int d \lambda_{i} e^{-N V\left(\lambda_{i}\right)} \sum_{n=0}^{N-1} p_{n}\left(\lambda_{i}\right) p_{n}\left(\lambda_{i}\right) \\
& =\sum_{n=0}^{N-1} \int d \lambda_{i} e^{-N V\left(\lambda_{i}\right)} p_{n}\left(\lambda_{i}\right) p_{n}\left(\lambda_{i}\right) \\
& =\sum_{n=0}^{N-1} \delta_{n, n} \\
\int K_{N}\left(\lambda_{i}, \lambda_{i}\right) d \lambda_{i} & =N
\end{aligned}
$$


Next, one can remark what happens if we integrate the determinant of the kernel over one of the eigenvalues, then we get

$$
\int \operatorname{det}\left(K_{N}\left(\lambda_{i}, \lambda_{j}\right)\right)_{i, j=1}^{k+1} d \lambda_{k+1}=\int\left|\begin{array}{ccc}
K_{N}\left(\lambda_{1}, \lambda_{1}\right) & \cdots & K_{N}\left(\lambda_{1}, \lambda_{k+1}\right) \\
K_{N}\left(\lambda_{2}, \lambda_{1}\right) & \cdots & K_{N}\left(\lambda_{2}, \lambda_{k+1}\right) \\
\vdots & \ddots & \vdots \\
K_{N}\left(\lambda_{k+1}, \lambda_{1}\right) & \cdots & K_{N}\left(\lambda_{k+1}, \lambda_{k+1}\right)
\end{array}\right| d \lambda_{k+1}
$$

by the Laplace formula which expresses the determinant of a matrix in terms of its minors

$$
\begin{aligned}
(*) & \equiv \int \operatorname{det}\left(K_{N}\left(\lambda_{i}, \lambda_{j}\right)\right)_{i, j=1}^{k+1} d \lambda_{k+1} \\
& =\int \sum_{i}^{k+1}(-1)^{k+1+i} K_{N}\left(\lambda_{k+1}, \lambda_{i}\right) \\
& \times\left|\begin{array}{ccc}
K_{N}\left(\lambda_{1}, \lambda_{1}\right) & \cdots & K_{N}\left(\lambda_{1}, \lambda_{k+1}\right) \\
K_{N}\left(\lambda_{2}, \lambda_{1}\right) & \cdots & K_{N}\left(\lambda_{2}, \lambda_{k+1}\right) \\
\vdots & \ddots & \vdots \\
K_{N}\left(\lambda_{k+1}, \lambda_{1}\right) & \cdots & K_{N}\left(\lambda_{k+1}, \lambda_{k+1}\right)
\end{array}\right| d \lambda_{k+1}^{(k+1, i)}
\end{aligned}
$$

where the minor $|\cdot|^{(i, k+1)}$ is the determinant of the $k \times k$ matrix that results from $|\cdot|$ by removing the row $i$ and the column $k+1$.

Now, expanding the minors, except the last one, along the last column by the 
Laplace formula

$(*)=\int \sum_{i}^{k}(-1)^{k+1+i} K_{N}\left(\lambda_{k+1}, \lambda_{i}\right) \sum_{j}^{k}(-1)^{j+k} \underbrace{K_{N}\left(\lambda_{j}, \lambda_{k+1}\right)}_{a_{j k}}$

$\times \underbrace{\left|\begin{array}{ccc}K_{N}\left(\lambda_{1}, \lambda_{1}\right) & \cdots & K_{N}\left(\lambda_{1}, \lambda_{k+1}\right) \\ K_{N}\left(\lambda_{2}, \lambda_{1}\right) & \cdots & K_{N}\left(\lambda_{2}, \lambda_{k+1}\right) \\ \vdots & \ddots & \vdots \\ K_{N}\left(\lambda_{k+1}, \lambda_{1}\right) & \cdots & K_{N}\left(\lambda_{k+1}, \lambda_{k+1}\right)\end{array}\right|}_{A^{(j k)}} d \lambda_{k+1}+$

$+\int(-1)^{2 k+2} K_{N}\left(\lambda_{k+1}, \lambda_{k+1}\right) \times$

$\times\left|\begin{array}{ccc}K_{N}\left(\lambda_{1}, \lambda_{1}\right) & \cdots & K_{N}\left(\lambda_{1}, \lambda_{k+1}\right) \\ K_{N}\left(\lambda_{2}, \lambda_{1}\right) & \cdots & K_{N}\left(\lambda_{2}, \lambda_{k+1}\right) \\ \vdots & \ddots & \vdots \\ K_{N}\left(\lambda_{k+1}, \lambda_{1}\right) & \cdots & K_{N}\left(\lambda_{k+1}, \lambda_{k+1}\right)\end{array}\right| \quad d \lambda_{k+1}$

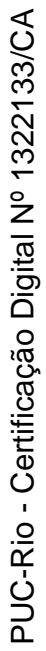

$=-\int \sum_{i}^{k} \sum_{j}^{k}(-1)^{i+j} K_{N}\left(\lambda_{k+1}, \lambda_{i}\right) K_{N}\left(\lambda_{j}, \lambda_{k+1}\right)$

$\times\left|\begin{array}{ccc}K_{N}\left(\lambda_{1}, \lambda_{1}\right) & \cdots & K_{N}\left(\lambda_{1}, \lambda_{k+1}\right) \\ K_{N}\left(\lambda_{2}, \lambda_{1}\right) & \cdots & K_{N}\left(\lambda_{2}, \lambda_{k+1}\right) \\ \vdots & \ddots & \vdots \\ K_{N}\left(\lambda_{k+1}, \lambda_{1}\right) & \cdots & K_{N}\left(\lambda_{k+1}, \lambda_{k+1}\right)\end{array}\right| \quad d \lambda_{k+1}+$

$+\int K_{N}\left(\lambda_{k+1}, \lambda_{k+1}\right)\left|\begin{array}{ccc}K_{N}\left(\lambda_{1}, \lambda_{1}\right) & \cdots & K_{N}\left(\lambda_{1}, \lambda_{k+1}\right) \\ K_{N}\left(\lambda_{2}, \lambda_{1}\right) & \cdots & K_{N}\left(\lambda_{2}, \lambda_{k+1}\right) \\ \vdots & \ddots & \vdots \\ K_{N}\left(\lambda_{k+1}, \lambda_{1}\right) & \cdots & K_{N}\left(\lambda_{k+1}, \lambda_{k+1}\right)\end{array}\right| \lambda_{k+1}^{(k+1, k+1)}$ 
seeing that the determinants are independent of $\lambda_{k+1}$, we find

$$
\begin{aligned}
& (*)=-\sum_{i}^{k} \sum_{j}^{k}(-1)^{i+j}\left|\begin{array}{ccc}
K_{N}\left(\lambda_{1}, \lambda_{1}\right) & \cdots & K_{N}\left(\lambda_{1}, \lambda_{k+1}\right) \\
K_{N}\left(\lambda_{2}, \lambda_{1}\right) & \cdots & K_{N}\left(\lambda_{2}, \lambda_{k+1}\right) \\
\vdots & \ddots & \vdots \\
K_{N}\left(\lambda_{k+1}, \lambda_{1}\right) & \cdots & K_{N}\left(\lambda_{k+1}, \lambda_{k+1}\right)
\end{array}\right| \\
& \times \int K_{N}\left(\lambda_{k+1}, \lambda_{i}\right) K_{N}\left(\lambda_{j}, \lambda_{k+1}\right) d \lambda_{k+1}+ \\
& +\left|\begin{array}{ccc}
K_{N}\left(\lambda_{1}, \lambda_{1}\right) & \cdots & K_{N}\left(\lambda_{1}, \lambda_{k+1}\right) \\
K_{N}\left(\lambda_{2}, \lambda_{1}\right) & \cdots & K_{N}\left(\lambda_{2}, \lambda_{k+1}\right) \\
\vdots & \ddots & \vdots \\
K_{N}\left(\lambda_{k+1}, \lambda_{1}\right) & \cdots & K_{N}\left(\lambda_{k+1}, \lambda_{k+1}\right)
\end{array}\right| \quad \int K_{N}\left(\lambda_{k+1}, \lambda_{k+1}\right) d \lambda_{k+1}
\end{aligned}
$$

by the properties of the kernel, we obtain

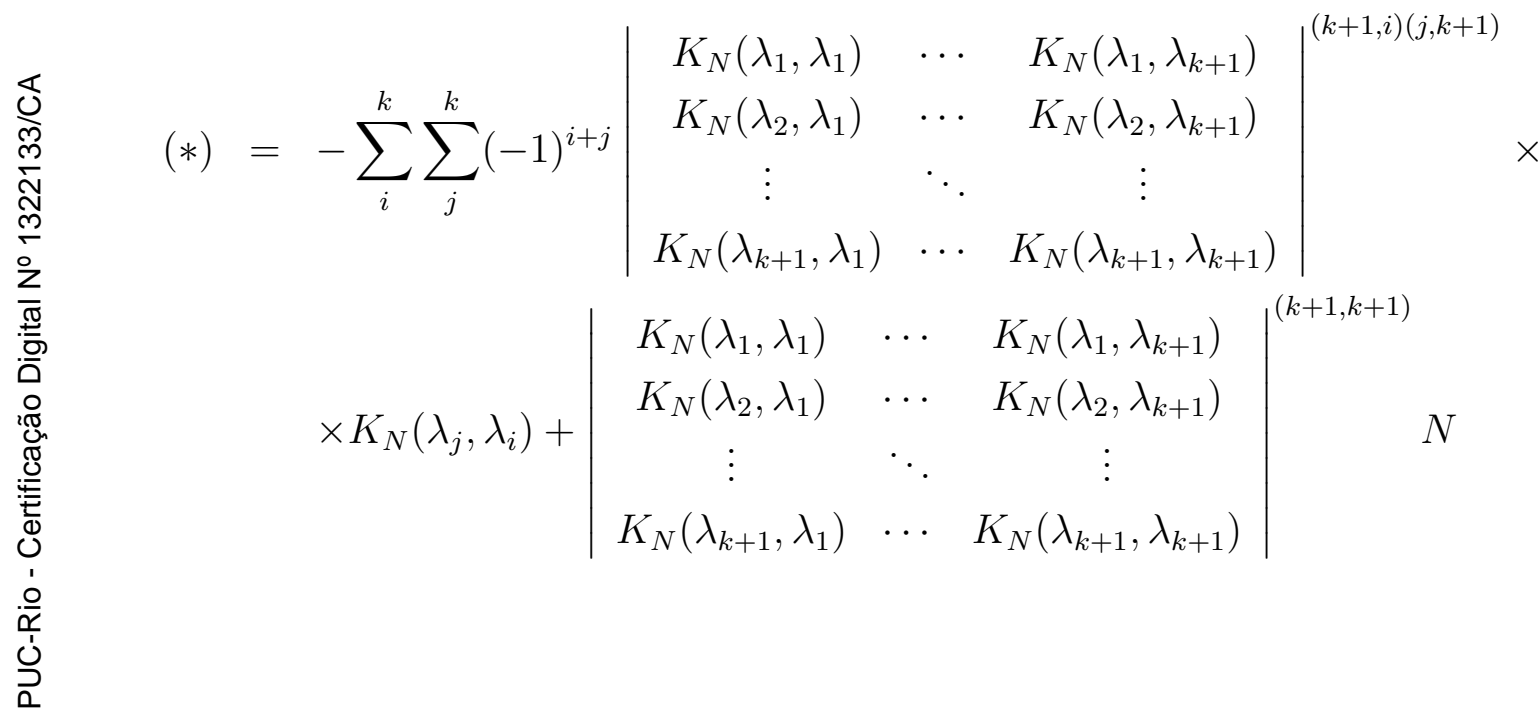


evaluating the sum over $j$ as a determinant, we have

$$
\begin{aligned}
& (*)=-\sum_{i}^{k}\left|\begin{array}{ccc}
K_{N}\left(\lambda_{1}, \lambda_{1}\right) & \cdots & K_{N}\left(\lambda_{1}, \lambda_{k+1}\right) \\
K_{N}\left(\lambda_{2}, \lambda_{1}\right) & \cdots & K_{N}\left(\lambda_{2}, \lambda_{k+1}\right) \\
\vdots & \ddots & \vdots \\
K_{N}\left(\lambda_{k+1}, \lambda_{1}\right) & \cdots & K_{N}\left(\lambda_{k+1}, \lambda_{k+1}\right)
\end{array}\right|^{(k+1, k+1)}+ \\
& +\left|\begin{array}{ccc}
K_{N}\left(\lambda_{1}, \lambda_{1}\right) & \cdots & K_{N}\left(\lambda_{1}, \lambda_{k+1}\right) \\
K_{N}\left(\lambda_{2}, \lambda_{1}\right) & \cdots & K_{N}\left(\lambda_{2}, \lambda_{k+1}\right) \\
\vdots & \ddots & \vdots \\
K_{N}\left(\lambda_{k+1}, \lambda_{1}\right) & \cdots & K_{N}\left(\lambda_{k+1}, \lambda_{k+1}\right)
\end{array}\right| \quad N \\
& =-k\left|\begin{array}{ccc}
K_{N}\left(\lambda_{1}, \lambda_{1}\right) & \cdots & K_{N}\left(\lambda_{1}, \lambda_{k+1}\right) \\
K_{N}\left(\lambda_{2}, \lambda_{1}\right) & \cdots & K_{N}\left(\lambda_{2}, \lambda_{k+1}\right) \\
\vdots & \ddots & \vdots \\
K_{N}\left(\lambda_{k+1}, \lambda_{1}\right) & \cdots & K_{N}\left(\lambda_{k+1}, \lambda_{k+1}\right)
\end{array}\right|(k+1, k+1) \\
& +N\left|\begin{array}{ccc}
K_{N}\left(\lambda_{1}, \lambda_{1}\right) & \cdots & K_{N}\left(\lambda_{1}, \lambda_{k+1}\right) \\
K_{N}\left(\lambda_{2}, \lambda_{1}\right) & \cdots & K_{N}\left(\lambda_{2}, \lambda_{k+1}\right) \\
\vdots & \ddots & \vdots \\
K_{N}\left(\lambda_{k+1}, \lambda_{1}\right) & \cdots & K_{N}\left(\lambda_{k+1}, \lambda_{k+1}\right)
\end{array}\right|
\end{aligned}
$$

resulting in

$$
\int \operatorname{det}\left(K_{N}\left(\lambda_{i}, \lambda_{j}\right)\right)_{i, j=1}^{k+1} d \lambda_{k+1}=(N-k) \operatorname{det}\left(K_{N}\left(\lambda_{i}, \lambda_{j}\right)\right)_{i, j=1}^{k}
$$




\section{C \\ Some considerations of the equivalence between orthogonal polynomials and RHP}

Here first of all we will prove with more detail the characterization of the orthogonal polynomials via RHP. Hence for $Y_{n, 11}$ by the first condition and the second condition of RHP,

$$
\left(Y_{n, 11}\right)_{+}(x)=\left(Y_{n, 11}\right)_{-}(x)
$$

we have that $Y_{n, 11}$ corresponds to a entire function. Furthermore, due to the third condition,

$$
Y_{n, 11}(z)=z^{n}+O\left(z^{n-1}\right)
$$

we obtain through the Liouville's theorem that $Y_{n, 11}$ is a monic polynomial of degree $\mathrm{n}, Y_{n, 11}=p_{n}$.

Next for $Y_{n, 12}$ by the first condition we have that $Y_{n, 12}$ is analytic in $\mathrm{C}^{+}$and $\mathrm{C}^{-}$. Further the second condition gives us that

$$
\left(Y_{n, 12}\right)_{+}(x)=\left(Y_{n, 11}\right)_{-}(x) w(x)+\left(Y_{n, 12}\right)_{-}(x) .
$$

Taking into count that $Y_{n, 11}=p_{n}$, we have that the preceding expression can be written on the following way

$$
\left(Y_{n, 12}\right)_{+}(x)=\left(Y_{n, 12}\right)_{-}(x)+p_{n}(x) w(x) .
$$

Therefore we have a scalar RHP for $Y_{n, 12}$ with the next conditions

1. $Y_{n, 12}$ is analytic in $\mathrm{C} \backslash \mathrm{R}$.

2. $\left(Y_{n, 12}\right)_{+}(x)=\left(Y_{n, 12}\right)_{-}(x)+p_{n}(x) w(x) . \quad x \in \mathrm{R}$

3. $Y_{n, 12} \rightarrow O\left(z^{-n-1}\right), \quad z \rightarrow \infty$

Then, the function $Y_{n, 12}$ that satisfies this conditions is given by the Cauchy transform of $p_{n}(x) w(x)$, on the following way

$$
Y_{n, 12}=\frac{1}{2 \pi i} \int_{\mathrm{R}} \frac{p_{n}(x) w(x)}{x-z} d x
$$


Appendix C. Some considerations of the equivalence between orthogonal polynomials and RHP

such that when $z \rightarrow \infty$ the asymptotic behaviour of $Y_{n, 12}$ is given by

$$
\begin{aligned}
Y_{n, 12} & =\frac{1}{2 \pi i} \int_{\mathrm{R}} \frac{p_{n}(x) w(x)}{z} \frac{1}{\frac{x}{z}-1} d x \\
& =-\frac{1}{2 \pi i} \int_{\mathrm{R}} \frac{1}{z}\left(\sum_{j=0}^{\infty}\left(\frac{x}{z}\right)^{j}\right) p_{n}(x) w(x) d x \\
& =-\frac{1}{2 \pi i} \sum_{j=0}^{\infty} \frac{1}{z^{j+1}} \int_{\mathrm{R}} x^{j} p_{n}(x) w(x) d x
\end{aligned}
$$

due to orthogonality relation the first $n$ terms of the sum are canceled

$$
Y_{n, 12}=-\frac{h_{n}}{2 \pi i z^{n+1}}-\sum_{j=n+2}^{\infty} \frac{1}{2 \pi i} \frac{1}{z^{j}} \int_{\mathrm{R}} x^{n+1} p_{n}(x) w(x) d x
$$

which as $z \rightarrow \infty$ is equivalent to

$$
Y_{n, 12}=O\left(z^{-n-1}\right)
$$

Analougosly as before to the first row of $Y_{n}$, for the second row we have that for $Y_{n, 21}$ by the Liouville's theorem is given by $Y_{n, 21}=-\frac{2 \pi i}{h_{n-1}} p_{n-1}$ and for $Y_{n, 22}$ we have a nonhomogeneus RHP with solution $Y_{n, 22}=-\frac{1}{h_{n-1}} \int_{\mathrm{R}} \frac{p_{n-1}(x) w(x) d x}{x-z}$. Therefore the solution $Y_{n}$ of the matricial RHP can be expressed as a function of orthogonal polynomials with respect to the measure $w(x) d x$ in $\mathrm{R}$.

Since by the second condition of the RHP the determinant of $Y_{n}$ satisfies the following relation

$$
\begin{aligned}
\operatorname{det} Y_{n+}(x) & =\operatorname{det} Y_{n-}(x) \operatorname{det}\left(\begin{array}{cc}
1 & w(x) \\
0 & 1
\end{array}\right) \\
& =\operatorname{det} Y_{n-}(x)
\end{aligned}
$$

Hence $\operatorname{det} Y_{n}$ corresponds to a entire function and by the third condition of RHP,

$$
\operatorname{det}\left(\begin{array}{cc}
z^{n}+O\left(z^{n-1}\right) & O\left(z^{-n-1}\right) \\
O\left(z^{n-1}\right) & z^{-n}+O\left(z^{-n-1}\right)
\end{array}\right) \cong 1
$$

Therefore, due to Liouville's theorem we have that

$$
\operatorname{det} Y_{n}(z)=1
$$

which means that every solution $Y_{n}$ of RHP is invertible and $Y_{n}^{-1}$ is analytic. Now we will show that $Y_{n}$ is unique, so we suppose that there are other solution 
Appendix C. Some considerations of the equivalence between orthogonal polynomials and RHP

$\tilde{Y}_{n}$ of RHP such that $X_{n}=\tilde{Y}_{n} Y_{n}^{-1}$ satisfies the following condition

$$
\begin{aligned}
X_{n+}(x) & =\tilde{Y_{n+}} Y_{n+}^{-1} \\
& =\tilde{Y_{n-}} j_{Y}(x) j_{Y}^{-1}(x) Y_{n-}^{-1} \\
& =\tilde{Y_{n-}} Y_{n-}^{-1} \\
& =X_{n-}(x)
\end{aligned}
$$

then $X_{n}$ is an entire function. Using the third condition of RHP we have as $z \rightarrow \infty$ that

$$
\begin{aligned}
& X_{n}(z) \cong\left(\begin{array}{cc}
z^{n}+O\left(z^{n-1}\right) & O\left(z^{-n-1}\right) \\
O\left(z^{n-1}\right) & z^{-n}+O\left(z^{-n-1}\right)
\end{array}\right)\left(\begin{array}{cc}
z^{-n}+O\left(z^{-n+1}\right) & O\left(z^{-n+1}\right) \\
O\left(z^{n+1}\right) & z^{n}+O\left(z^{n+1}\right)
\end{array}\right) \\
& X_{n}(z) \cong I
\end{aligned}
$$

So by the Liouville's theorem we have, $X_{n}(z)=I$ which means that $\tilde{Y}_{n} Y_{n}^{-1}=I$, for this reason

$$
\tilde{Y}_{n}=Y_{n}
$$

which proves the uniqueness of $Y_{n}$.

Now we will find the coefficients of the three term recurrence relation for $n>0$

$$
\lambda p_{n}(\lambda)=p_{n+1}(\lambda)+s_{n} p_{n}(\lambda)+r_{n} p_{n-1}(\lambda)
$$

as function of the RHP's solution. To our pourpose we multiply both the second and third condition of the RHP by $\left(\begin{array}{cc}z^{-n} & 0 \\ 0 & z^{n}\end{array}\right)$ to compare them. Hence for the second condition we have

$$
Y_{n}(z)\left(\begin{array}{cc}
z^{-n} & 0 \\
0 & z^{n}
\end{array}\right)=\left(\begin{array}{cc}
z^{-n} p_{n}(z) & \frac{z^{n}}{2 \pi i} \int_{\mathrm{R}} \frac{p_{n}(x) w(x) d x}{x-z} \\
-\frac{2 z^{-n} \pi i}{h_{n-1}} p_{n-1}(z) & -\frac{z^{n}}{h_{n-1}} \int_{\mathrm{R}} \frac{p_{n-1}(x) w(x) d x}{x-z}
\end{array}\right)
$$

and for the third condition we have

$$
\begin{aligned}
Y_{n}(z)\left(\begin{array}{cc}
z^{-n} & 0 \\
0 & z^{n}
\end{array}\right) & =\left(\left(\begin{array}{cc}
z^{n} & 0 \\
0 & z^{-n}
\end{array}\right)+\sum_{k=1}^{\infty} Y_{k}\left(\begin{array}{cc}
z^{n-k} & z^{-n-k} \\
z^{n-k} & z^{-n-k}
\end{array}\right)\right)\left(\begin{array}{cc}
z^{-n} & 0 \\
0 & z^{n}
\end{array}\right) \\
& =I+\sum_{k=1}^{\infty} \frac{Y_{k}}{z^{k}}
\end{aligned}
$$

and by comparing (C.2) and (C.3), we obtain that

$$
I+\sum_{k=1}^{\infty} \frac{Y_{k}}{z^{k}}=\left(\begin{array}{cc}
z^{-n} p_{n}(z) & \frac{z^{n}}{2 \pi i} \int_{\mathrm{R}} \frac{p_{n}(x) w(x) d x}{x-z} \\
-\frac{2 z^{-n} \pi i}{h_{n-1}} p_{n-1}(z) & -\frac{z^{n}}{h_{n-1}} \int_{\mathrm{R}} \frac{p_{n-1}(x) w(x) d x}{x-z}
\end{array}\right)
$$


Appendix C. Some considerations of the equivalence between orthogonal polynomials and RHP

If we consider that

$$
\begin{gathered}
p_{n}(z)=z^{n}+a_{n-1} z^{n-1}+a_{n-2} z^{n-2}+\ldots \\
p_{n-1}(z)=z^{n-1}+b_{n-2} z^{n-2}+b_{n-3} z^{n-3}+\ldots
\end{gathered}
$$

so by replacing these expressions in the first column and expanding the second column of the right term in (C.4), we have

$$
I+\sum_{k=1}^{\infty} \frac{Y_{k}}{z^{k}}=\left(\begin{array}{cc}
z^{-n}\left(z^{n}+a_{n-1} z^{n-1}+\ldots\right) & \frac{z^{n}}{2 \pi i} \int_{\mathrm{R}} p_{n}(x) w(x) \sum_{j=0}^{\infty} \frac{x^{j}}{z^{j+1}} d x \\
-\frac{2 z^{-n} \pi i}{h_{n-1}}\left(z^{n-1}+b_{n-2} z^{n-2}+\ldots\right) & -\frac{z^{n}}{h_{n-1}} \int_{\mathrm{R}} p_{n-1}(x) w(x) \sum_{j=0}^{\infty} \frac{x^{j}}{z^{j+1}} d x
\end{array}\right)
$$

by the orthogonality relation we have that

$$
\begin{aligned}
(* *) & =\left(\begin{array}{cc}
1+\frac{a_{n-1}}{z}+\frac{a_{n-2}}{z^{2}}+O\left(z^{-3}\right) & z^{n}\left(-\frac{h_{n}}{2 \pi i z^{n+1}}+O\left(z^{-n-2}\right)\right) \\
-\frac{2 \pi i}{h_{n-1} z}-\frac{2 \pi b_{n-2} i}{h_{n-1} z^{2}}-\frac{2 \pi b_{n-3} i}{h_{n-1} z^{3}}+O\left(z^{-4}\right) & z^{n}\left(\frac{h_{n-1}}{h_{n-1} z^{n}}+O\left(z^{-n-1}\right)\right)
\end{array}\right) \\
& =\left(\begin{array}{cc}
1+\frac{a_{n-1}}{z}+\frac{a_{n-2}}{z^{2}}+O\left(z^{-3}\right) & -\frac{h_{n}}{2 \pi i z}+O\left(z^{-2}\right) \\
-\frac{2 \pi i}{h_{n-1} z}-\frac{2 \pi b_{n-2} i}{h_{n-1} z^{2}}-\frac{2 \pi b_{n-3} i}{h_{n-1} z^{3}}+O\left(z^{-4}\right) & 1+O\left(z^{-1}\right)
\end{array}\right)
\end{aligned}
$$

If we decompose this expression like the left side of (C.4) we obtain

$$
\begin{aligned}
I+\frac{Y_{1}}{z}+\frac{Y_{2}}{z^{2}}+\sum_{k=3}^{\infty} \frac{Y_{k}}{z^{k}}= & \left(\begin{array}{ll}
1 & 0 \\
0 & 1
\end{array}\right)+\left(\begin{array}{cc}
a_{n-1} & -\frac{h_{n}}{2 \pi i} \\
-\frac{2 \pi i}{h_{n-1}} & O(1)
\end{array}\right) \frac{1}{z}+\left(\begin{array}{cc}
a_{n-2} & O(1) \\
-\frac{2 \pi b_{n-2} i}{h_{n-1}} & O(1)
\end{array}\right) \frac{1}{z^{2}}+ \\
& +\left(\begin{array}{cc}
O(1) & O(1) \\
-\frac{2 \pi b_{n-3} i}{h_{n-1}} & O(1)
\end{array}\right) \frac{1}{z^{3}}+O\left(z^{-4}\right)
\end{aligned}
$$

By comparing each order we have

$$
\begin{gathered}
Y_{1}=\left(\begin{array}{cc}
a_{n-1} & -\frac{h_{n}}{2 \pi i} \\
-\frac{2 \pi i}{h_{n-1}} & O(1)
\end{array}\right), \\
Y_{2}=\left(\begin{array}{cc}
a_{n-2} & O(1) \\
-\frac{2 \pi b_{n-2} i}{h_{n-1}} & O(1)
\end{array}\right) \\
Y_{3}=\left(\begin{array}{cc}
O(1) & O(1) \\
-\frac{2 \pi b_{n-3} i}{h_{n-1}} & O(1)
\end{array}\right)
\end{gathered}
$$

Now we evaluate the coefficients of the recurrence relation by the elements of these matrices. First, to obtain $r_{n}$ from $Y_{1}$ we find the following relation

$$
Y_{1,21} Y_{1,12}=\left(-\frac{2 \pi i}{h_{n-1}}\right)\left(-\frac{h_{n}}{2 \pi i}\right)=\frac{h_{n}}{h_{n-1}}=r_{n}
$$


Appendix C. Some considerations of the equivalence between orthogonal polynomials and RHP

where the last equality is given by (4.26). Finally to obtain $s_{n-1}$ we unfold the polynomials in the recurrence relation by (C.1) in the following way

$$
\begin{gathered}
z\left(z^{n-1}+b_{n-2} z^{n-2}+b_{n-3} z^{n-3}+\ldots\right)=\left(z^{n}+a_{n-1} z^{n-1}+a_{n-2} z^{n-2}+\ldots\right) \\
+s_{n-1}\left(z^{n-1}+b_{n-2} z^{n-2}+b_{n-3} z^{n-3}+\ldots\right)+O\left(z^{n-2}\right)
\end{gathered}
$$

classifying order by order we obtain

$$
z^{n}+b_{n-2} z^{n-1}+b_{n-3} z^{n-2}+\ldots=z^{n}+\left(a_{n-1}+s_{n-1}\right) z^{n-1}+O\left(z^{n-2}\right)
$$

hence from the $O\left(z^{n-1}\right)$ term we have

$$
s_{n-1}=b_{n-2}-a_{n-1}
$$

note that from the matrix elements of $Y_{1}$ and $Y_{2}$ we find that

$$
\frac{Y_{2,21}}{Y_{1,21}}-Y_{1,11}=b_{n-2}-a_{n-1}=s_{n-1}
$$

Now we express the correlation kernel in terms of the solution of RHP. Since $\operatorname{det} Y_{n}(z)=1$ the inverse of the solution of RHP is given by

$$
Y_{n}^{-1}(z)=\left(\begin{array}{cc}
-\frac{1}{h_{n-1}} \int_{\mathrm{R}} \frac{p_{n-1}(x) w(x) d x}{x-z} & -\frac{1}{2 \pi i} \int_{\mathrm{R}} \frac{p_{n}(x) w(x) d x}{x-z} \\
\frac{2 \pi i}{h_{n-1}} p_{n-1}(z) & p_{n}(z)
\end{array}\right)
$$

hence by multiplying by $\left(\begin{array}{ll}0 & 1\end{array}\right)$ we obtain

$$
\left(\begin{array}{ll}
0 & 1
\end{array}\right) Y_{N+}^{-1}\left(\lambda_{j}\right)=\left(\frac{2 \pi i}{h_{N-1}} p_{N-1}\left(\lambda_{j}\right) p_{N}\left(\lambda_{j}\right)\right)
$$

while by multiplying $Y_{N-}\left(\lambda_{i}\right)$ by $\left(\begin{array}{l}1 \\ 0\end{array}\right)$ we obtain

$$
Y_{N-}\left(\lambda_{i}\right)\left(\begin{array}{c}
1 \\
0
\end{array}\right)=\left(\begin{array}{c}
p_{N}\left(\lambda_{i}\right) \\
-\frac{2 \pi i}{h_{N-1}} p_{N-1}\left(\lambda_{i}\right)
\end{array}\right)
$$

therefore

$$
\left(\begin{array}{ll}
0 & 1
\end{array}\right) Y_{N+}^{-1}\left(\lambda_{j}\right) Y_{N-}\left(\lambda_{i}\right)\left(\begin{array}{l}
1 \\
0
\end{array}\right)=\frac{2 \pi i}{h_{N-1}}\left(p_{N-1}\left(\lambda_{j}\right) p_{N}\left(\lambda_{i}\right)-p_{N}\left(\lambda_{j}\right) p_{N-1}\left(\lambda_{i}\right)\right)
$$


Appendix C. Some considerations of the equivalence between orthogonal polynomials and RHP

hence the correlation kernel is given by

$$
K_{N}\left(\lambda_{i}, \lambda_{j}\right)=\frac{e^{-\frac{1}{2} N\left(V\left(\lambda_{i}\right)+V\left(\lambda_{j}\right)\right)}}{2 \pi i\left(\lambda_{i}-\lambda_{j}\right)}\left(\begin{array}{ll}
0 & 1
\end{array}\right) Y_{N+}^{-1}\left(\lambda_{j}\right) Y_{N-}\left(\lambda_{i}\right)\left(\begin{array}{l}
1 \\
0
\end{array}\right)
$$




\section{D \\ Universality}

A general phenomena so-called Universality allows the different applications in mathematics, physics and science in general. In RMT there is a lot of progress about this phenomena which for a family of random matrices the limiting distribution of various statistics of the eigenvalue spectrum determined by the kernel after a proper scaling converges to a common distribution as $N \rightarrow \infty$, farther the kernel of this distribution is non random which is connect to some kernels. But is not complete its understanding yet.

In the scalar RMT this phenomena is known as central limit theorem which states that for a group of mutually independent random variables $X_{1}, X_{2}, \ldots, X_{N}$, no matter what distribution characterize this random variables, so the normalized average in the limit $N \rightarrow \infty$ is given by a Gaussian distribution, which have special properties as?.

A family of the local distribution of eigenvalues in the matrix is detremined by the correlation kernel. Dyson found an exact solution for the scaling limit of correlation kernel between eigenvalues in the Gaussian unitary ensemble. Now we know that the majority of nongaussian unitary ensembles below to the same family of the Gaussian unitary ensemble which this family is determined by the scaling limit of the correlation kernel inside the support of the limiting spectral measure,

$$
K(u, v)=\frac{\sin (\pi(u-v))}{\pi(u-v)}
$$

and at the edge point of the spectral measure by

$$
K(u, v)=\frac{A i(u) A i^{\prime}(v)-A i^{\prime}(u) A i(v)}{u-v}
$$

But for the rest of nongaussian unitary ensembles this last kernel is different wich is given by the criticality of the potential in this point wich is related to the double scaling limit, while for this rest of nongaussian unitary ensembles the kernel inside the support is the same in the case of polynomial potentials. In this way for each family of Random matrix one can find a type of kernel. Hence in the unitary ensemble by Riemann hilbert techniques one can classify this families by the critical points of the support. Because correlation kernel 
for finite $N$ can be expres in terms of orthogonal polynomials on the line. 


\section{Bibliography}

[1] M.L. Mehta, Random Matrices, Academic Press, New York, 1967; 3rd Edition, Elsevier, Amsterdam, 2004.

[2] M.J. Ablowitz, A.S. Fokas, Complex variables: Introduction and Applications, Cambridge University Press, 1997.

[3] P. Ginsparg and G. Moore, Lectures on 2D Gravity and 2D String Theory, hep-th/9304011v1.

[4] F. David, C. Hadendorf, and K.J. Wiese, A growth model for RNA secondary structures, J. Stat. Mech., (2008) P04008,0711.3421.

[5] H.L. Montgomery, The pair correlation of zeros of the zeta function, Proc. Sympos. Pure Math. Vol. XXIV. St. Louis Univ. St. Louis Mo., 1972.

[6] E. Brezin, C. Itzykson, G. Parisi, J.B. Zuber, Planar Diagrams, Commun. Math. Phys. 59 (1978) 35.

[7] C. A. Tracy and H. Widom, The Distributions of Random Matrix Theory and their Applications, New Trends in Mathematical Physics, ed. V. pp. 753-765.

[8] M.L. Metha, A method of integration over matrix variables, Commun. Math. Phys. 79 (1981) 327-340.

[9] E.P. Wigner Ann. Math. 53 (1951) 36.

[10] E.P. Wigner, On the statistical distribution of the widhts and spacings of nuclear resonance levels, Proc. Cambridge Phil. Soc. 47 (1951) 790.

[11] P. Di Francesco, 2D quantum gravity, matrix models and graph combinatorics, math-ph/0406013.

[12] G.W. Anderson, A. Guionnet,O. Zeitouni, An Introduction to Random Matrices, Cambridge Studies in Advanced Mathematics 2009

[13] P. Deift, Orthogonal polynomials and random matrices: a Riemann-Hilbert approach, V. 3, AMS Bookstore 1999. 
[14] G. Szego, Orthogonal Polynomials, v. 23, 9780821889527, American mathematical society 1939 .

[15] T.S. Chihara, An Introduction to Orthogonal Polynomials, 9780486479293, Dover Publications 2011.

[16] A.B.J. Kuijlaars, Riemann-Hilbert analysis for orthogonal polynomials, Orthogonal Polynomials and Special Functions, E. Koelink, W. Van Assche, (eds), Lecture Notes in Mathematics, Vol. 1817, Berlin-Heidelberg-New York: SpringerVerlag, 2003, pp. 167-210

[17] P.M. Bleher, Lectures on random matrix models. The Riemann-Hilbert approach,

[18] A. Fokas, A. Its, A. Kitaev, The isomonodromy approach to matrix models in 2D quantum gravity, Commun. Math. Phys. 1473951992.

[19] P. Deift, T. Kriecherbauer, K.T.R. McLaughlin, S. Venakides, X. Zhou, Uniform asymptotics for polynomials orthogonal with respect to varying exponential weights and applications to universality questions in random matrix theory, Communications on Pure and Applied Mathematics 52 (11), 1335-1425.

[20] P. Deift, T. Kriecherbauer, K.T.R. McLaughlin, S. Venakides, X. Zhou, Strong asymptotics of orthogonal polynomials with respect to exponential weights, Communications on Pure and Applied Mathematics 52 (12), 1491-1552

[21] P. Di Francesco, P. Ginsparg and J. Zinn-Justin, 2D Gravity and Random Matrices, Phys. Rept., 254 (1995) 1-133, hep-th/9306153v2.

[22] J. Ambjorn, Quantization of Geometry, hep-th/9411179.

[23] S. Zohren, A Causal Perspective on Random Geometry, 0905.0213.

[24] S. Zohren, Analytic Results in 2D Causal Dynamical Triangulations: A Review, hep-th/0609177v1.

[25] F.J. Dyson, Statistical Theory of the Energy Levels of Complex Systems. I, J. Math. Phys., 3 (1962) 140-156.

[26] G. Akemann, J. Baik and P. Di Francesco, The Oxford Handbook of Random Matrix Theory, Oxford University Press, 2011.

[27] M. Marino, Les Houches Lectures on Matrix Models and Topological Strings, hep-th/0410165v3. 
[28] G. 't Hooft, A Planar Diagram Theory for Strong Interactions, Nucl. Phys., B72 (1974) 461.

[29] D. Bessis, C. Itzykson, and J.B. Zuber, Quantum Field Theory Techniques in Graphical Enumeration, Adv. Appl. Math., 1 (1980) 109-157.

[30] J. Ambjorn, B. Durhuus and T. Jonsson, Quantum Geometry: A Statistical Field Theory Approach, Cambridge University Press, Cambridge, UK, 1997.

[31] B. Eynard, Random Matrices, Saclay-IPht, 2000.

[32] M. Marino, Lectures on Non-perturbative Effects in Large N Gauge Theories, Matrix Models and Strings, Fortsch. Phys., 62 (2014) 455-540, 1206.6272. 